San Jose State University

SJSU ScholarWorks

Master's Theses

Master's Theses and Graduate Research

1998

\title{
Evaluation of the architectural design for digital camera applications
}

Paul Yang Moua

San Jose State University

Follow this and additional works at: https://scholarworks.sjsu.edu/etd_theses

\section{Recommended Citation}

Moua, Paul Yang, "Evaluation of the architectural design for digital camera applications" (1998). Master's Theses. 1654.

DOI: https://doi.org/10.31979/etd.j55q-xdt7

https://scholarworks.sjsu.edu/etd_theses/1654

This Thesis is brought to you for free and open access by the Master's Theses and Graduate Research at SJSU ScholarWorks. It has been accepted for inclusion in Master's Theses by an authorized administrator of SJSU ScholarWorks. For more information, please contact scholarworks@sjsu.edu. 


\section{INFORMATION TO USERS}

This manuscript has been reproduced from the microfilm master. UMI films the text directly from the original or copy submitted. Thus, some thesis and dissertation copies are in typewriter face, while others may be from any type of computer printer.

The quality of this reproduction is dependent upon the quality of the copy submitted. Broken or indistinct print, colored or poor quality illustrations and photographs, print bleedthrough, substandard margins, and improper alignment can adversely affect reproduction.

In the unlikely event that the author did not send UMI a complete manuscript and there are missing pages, these will be noted. Also, if unauthorized copyright material had to be removed, a note will indicate the deletion.

Oversize materials (e.g., maps, drawings, charts) are reproduced by sectioning the original, beginning at the upper left-hand comer and continuing from left to right in equal sections with small overlaps. Each original is also photographed in one exposure and is included in reduced form at the back of the book.

Photographs included in the original manuscript have been reproduced xerographically in this copy. Higher quality 6" $\times 9$ " black and white photographic prints are available for any photographs or illustrations appearing in this copy for an additional charge. Contact UMI directly to order.

\section{UMI}

A Bell \& Howell Information Company

300 North Zeeb Road, Ann Aubor MI 48106-1346 USA

$313 / 761-4700 \quad 800 / 521-0600$ 


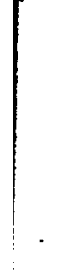


EVALUATION OF THE ARCHITECTURAL DESIGN FOR DIGITAL CAMERA APPLICATIONS

\author{
A Thesis \\ Presented to \\ The Faculty of Computer, Information, and System Engineering \\ San Jose State University
}

\author{
In Partial Fulfillment \\ of the Requirements for the Degree \\ Master of Science
}

by

Paul Yang Moua

May 1998 
UMI Number: 1389665

UMI Microform 1389665

Copyright 1998, by UMI Company. All rights reserved.

This microform edition is protected against unauthorized copying under Title 17, United States Code.

\section{UMI}

300 North Zeeb Road

Ann Arbor, MI 48103 
C 1998

Paul Yang Moua

ALL RIGHTS RESERVED 
APPROVED FOR THE DEPARTMENT OF COMPUTER, INFORMATION, AND SYSTEM ENGINEERING

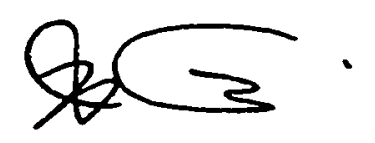

Dr. Hua Harry Li

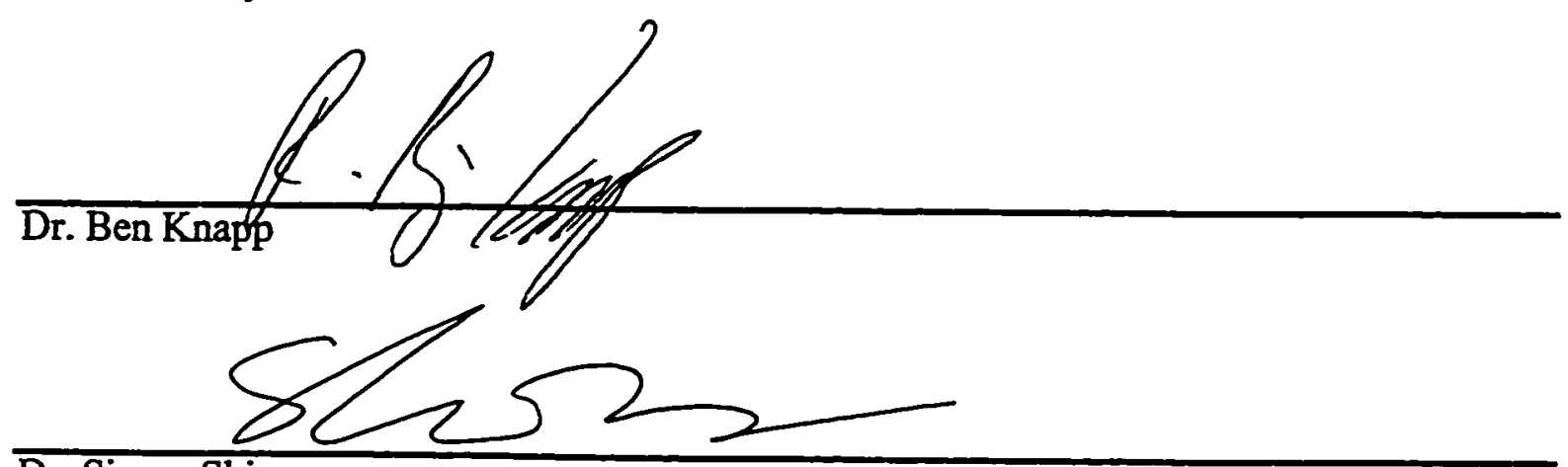

Dr. Simon Shim

APPROVED FOR THE UNIVERSITY

Willim Zishe 


\begin{abstract}
EVALUATION OF ARCHITECTURAL DESIGN FOR DIGITAL CAMERA APPLICATIONS
\end{abstract}

By Paul Yang Moua

The digital camera is in its booming stage of development. Many companies both in the software, hardware, and digital imaging fields are plunging onto the digital camera bandwagon. This thesis surveys the core technologies that make digital cameras possible in the consumer market today. These core technologies include CCD image capture, image compression techniques, convergence of embedded DSP processor and RISC processor, high speed I/O design and memory storage device. We compare some of the general-purpose architectural designs from various manufacturers that include most of these features. Furthermore, we propose an architectural design that is suitable for both still camera and motion camera applications with state-of-the-art multimedia I/O design features. Lastly, we simulate a 16-bit RISC core processor in HDL. The simulation is a subset of the R2000 MIPS architecture that has instruction sets relevant to digital camera applications. 


\section{ACKNOWLEDGEMENTS}

The opportunity to formally thank all those who have made an impact on a Thesis comes all too infrequently, so I shall not be brief.

Intellectually, I owe thanks to Dr. Hua Harry Li who served as my Thesis Advisor. He has been proof reading and giving suggestions throughout the whole course of the Thesis. Also, I owe thanks to Dr. Simon Shim and Dr. Ben Knapp who served as the Thesis committees. Furthermore, I owe thanks to Paj Yaj, Lisa Thao, and Kabao Moua for their proof reading the script before it actually submitted to the committee.

Perhaps most of all, I owe thanks to my parents, Mee Yang and Kaneng Moua, who instilling in me a desire to learn. Also, I owe thanks to my dad and Corrine Hung for their support in taking care of my rentals while I was busy with my studies.

Even an abundance of financial and intellectual support does not lead to result without emotional and spiritual support. For these supports, I am indebted to God. He has helped my mom recovering from two strokes during the course of my graduate studies. 


\section{TABLE OF CONTENTS}

ACKNOWLEDGMENT

LIST OF TABLES ix

LIST OF FIGURES Xi

CHAPTER

I. INTRODUCTION 1

1.1 Background 1

1.2 Problem Statement 3

1.3 Objectives of This Study 4

II. LITERATURE SURVEY 7

2.1 The State-of-the-Art Technology in Digital Camera Applications 7

2.2 Digital Camera System 9

$\begin{array}{ll}2.2 .1 \text { Capture Mode } & 15\end{array}$

$\begin{array}{lll}2.2 .2 & \text { Display Mode } & 16\end{array}$

$\begin{array}{ll}\text { 2.2.3 Digital Camera CODEC } & 17\end{array}$

2.2.3.1 JPEG Standard 17

2.2.3.2 IC CODEC $2 \mathrm{I}$

2.3 Digital Camera External Accessories $\quad 22$

2.3.1 External Memory $\quad 22$

2.3.2 I/O Interface $\quad 24$

2.3.3 Software Applications $\quad 24$

III. EVALUATION OF THE MICROPROCESSOR 25

ARCHITECTURE DESIGN

3.1 The Architecture Design $\quad 25$

3.1.1 Matsushita VDSP2 Architecture 26

3.1.2 Hitachi SH-DSP Architecture 28

3.1.2.1 SH-DSP CPU 28

3.1.3 LSI Logic DCAM-101 Architecture 31

3.1.3.1 Processor Unit 31

3.1.3.2 JPEG CODEC Unit 32

3.1.4 Motorola MPC823 Architecture $\quad 32$

3.1.4.1 PowerPC Processor Core 33

3.1.4.2 MPC823 BIU 34

3.1.5 TI TMS320C80 Architecture 36

3.1.5.1 MP Architecture 36 
3.1.5.2 DSP Architecture 39

3.1.5.3 Transfer Controller Architecture $\quad 40$

3.1.5.4 Crossbar Architecture $\quad 43$

3.2 The Instruction Set 46

3.3 The I/O Interface Design 48

3.3.1 Matsushita VDSP2 48

3.3.2 Hitachi SH-DSP 48

3.3.3 LSI Logic DCAM-101 Interface Unit 49

3.3.4 Motorola MPC823 50

3.3.4.1 MPC823 Communication Processor Module $\quad 50$

3.3.4.2 MPC823 LCD Unit 51

3.3.4.3 MPC823 Video Unit 51

3.3.5 TI TMS320C80 Video Unit 54

3.4 The Benchmarks of the Performance 55

IV. THE SUGGESTED ARCHITECTURE FOR HIGH SPEED HIGH 58 PERFORMACE DIGITAL CAMERA

4.1 The Architectural Overview 58

4.1.1 The Suggested CODEC Unit 60

4.1.2 The Suggested CPM Unit 69

4.1.3 The Suggested CPU Unit 73

4.2 The Performance $\quad 74$

4.2.1 General Computational Capability 74

4.2.2 Potential for Big family of Signal Processing Functions 74

4.3 Evaluation of the Need to Support to Modern Operating Systems 75

V. VHDL SIMULATIONS

5.I MIPS Overview $\quad 76$

5.1.1 Start-up Requirements $\quad 76$

5.1.2 Instruction and Instruction Formats 77

5.2 The Top Level Design and Simulation 80

5.3 The Subsystem and Basic Building Block Design and Simulation $\quad 82$

$\begin{array}{lr}\text { 5.3.1 PC Module } & 82\end{array}$

5.3.2 Memory Module $\quad 82$

5.3.3 Pipelines $\quad 83$

5.3.4 Register File Module $\quad 83$

5.3.5 16-bit ALU Module $\quad 87$

5.3.6 Control Module $\quad 90$

5.3.7 Hazard Modules $\quad 92$

$\begin{array}{ll}5.4 \text { The Evaluations } & 95\end{array}$

VI. CONCLUSION $\quad 99$ 
REFERENCES

APPENDIX 


\section{LIST OF TABLES}

2.1 Category of digital camera by price.

$\begin{array}{lll}2.2 & \text { List of high-range digital cameras with some key features. } & 8\end{array}$

$\begin{array}{lll}2.3 & \text { The luminance quantization table [9]. } & 20\end{array}$

2.4 The chrominance quantization table [9]. 20

2.5 Commercially available ICs for JPEG. 22

2.6 PCMCIA Memory Cards available for various digital cameras 23

3.1 The TMS320C80 Crossbar's priority arrangement use to schedule 45 task. The priority scheduling algorithm is designed to allow TC pipeline Flush, VC packet transfers (PTs), external PTs, cache, DEA and Urgent PTs to have the highest priority among all tasks. The TC low-priority PTs have the lowest priority.

3.2 Show the different classes of instructions.

3.3 A comparison of the performance for the evaluated processors. Also, 56 shown is the technology used to fabricate the processors and the maximum clock rate for each individual processor.

3.4 Execution time for MPEG2 encoder using Matsushita Electric's VDSP2 processor [25-26].

3.5 An illustration of the processors' MIPS bandwidth to do image processing. The illustration is based on $640 \times 480$ image at 30 frames per second. Based on this, the VDSP2 has the highest MIPS bandwidth for image processing.

4.1 The sizes of each subsample in MPEG-2. The $Y$ component is the same of all three subsamples. The $\mathrm{Cr}$ and $\mathrm{Cb}$ components reduced according to the ratios.

5.1 The instruction sets that support in the XCAM-2000 CPU simulation. These instruction sets are subset of the MIPS R2000 instructions.

5.2 The functions implemented in the ALU Module of the XCAM-2000. 
5.3 The control signals that are used in each stage of the pipeline along with their type of instruction.

5.4 The test case for various instructions in the XCAM-2000 CPU 96

5.5 The test case for LW and SW instructions in XCAM-2000 CPU. 96 The first SW stores 6 into memory location 3. The LW reads 6 From the same memory 


\section{LIST OF FIGURES}

2.1 The major blocks of a typical digital camera. The overall blocks are classified into imager block, signal processor/system block, and external media block.

2.2 A typical diagram of CCD design [34]. The optical black is used to reference how much darkness is applied during the capturing process. Both the active pixel area and the effective pixel area are used to store the image. However, only the effective pixel area is valid for display.

2.3 A Bayer pattern arrangement of RGB color for one-exposure CCD. Both the active pixel area and the affective pixel are arranged with the Bayer pattern.

2.4 The capturing sequence of an image.

2.5 The displaying sequence of a digital camera.

2.6 Major blocks of JPEG encoding standard.

3.1 Block diagram of the Matsushita Electric Industrial's VDSP2 processor.

3.2 Block diagram of the Hitachi's SH-DSP HD643710F processor [29].

3.3 Block diagram of the LSI Logic's DCAM-10 I processor [30].

3.4 Block diagram of the Motorola's MPC 823 processor [27].

3.5 Block diagram of the MPC 823's PowerPC Core architectural design [27].

3.6 Block diagram of the TI's TMS320C80 processor [28].

3.7 The IEEE floating-point standard format. 38

3.8 Block diagram of the TMS320C80's DSP architectural design [28]. 41

3.9 The parameter table use by the TMS320C80's TC to handle data movement. (a) Shows the 64-byte use for long packet. (b) Shows the 16-byte use for short packet [28]. 
3.10 An ideal crossbar topology interconnection for $\mathrm{N}$ processors to $\mathrm{M}$ memory units.

3.11 The round-robin's token passing order that is used to resolve conflict among the DSPs.

3.12 Block diagram of the MPC823's LCD controller architectural design.

4.1 The suggested architecture's functional block diagram.

4.2 Block diagram of video compression in the JPEG and MPEG International Standards. The dotted blocks are the JPEG standard. Both the dotted blocks and solid blocks are the MPEG standard.

4.3 Macroblock of the luminance and chrominance in the MPEG's subsample. Each square corresponds to $8 \times 8$ square pixels. The diagram shows the relationship of the squares to its subsample ratios for the luminance and chrominance components.

4.4 The process to find the motion vector using a full search algorithm.

(a) Shows the macroblock in the current frame. (b) Shows the search region that is defined by $[-p, p]$ in the reference frame.

(c) Shows the motion vector as a displacement between the current macroblock and the best possible macroblock in the reference frame.

4.5 Motion compensation for the $\mathrm{P}$-frame. The prediction error can be subtracted from either I-frame.

4.6 Motion compensation for the B-frame. The prediction error can be subtracted from either the P-frame or the I-frame or average of both the P-frame and I-frame.

4.7 The USB bus topology [37].

4.8 The USB's connection establishment between the device and the Host.

5.1 The three types of instruction format in the MIPS R2000 instruction sets. (a) Shows the R-type breakdown of the 16-bits. (b) Shows the I-type breakdown of the 16-bits. (c) Shows the J-type breakdown of the 16-bits.

5.2 The interconnected of all the modules in the 16-bit CPU. The 
bold lines represent buses with two or more bits. The thin lines represent signals with only one bit.

5.3 Design of the program counter. It uses sixteen $\mathrm{D}$ flip-flop registers connecting in parallel. Upon clocking, the data will be latched into the register. Reset signal will clear the content of the 16 registers.

5.4 Design of the memory module. Each register is designed from sixteen D flip-flops connecting in parallel. For illustration purposes, the design has only thirty-one registers. Ideally, it can decode up to 65K address locations. The RD_Address bus and the RD_signal signal are both used to select the data from register to be put out.

5.5 The design of the register file. It consists of seven registers with D flip-flop connecting in parallel. The write address will decode which register to write to. The read of DATA 1 and DATA 2 is a multiplexer (MUX) design that makes selection of which register to read from.

5.6 ALU Design. The status flag is generated by using both input A, B and the result of the function.

5.7 The state machine to generate all the control signals in the XCAM-2000 CPU. In this diagram, VCC and "l" both means logic high.

5.8 Structural hazards occur when LW or SW instruction and R-type instruction try to write to the same location at the same time. The LW or SW instruction reference memory so it has 5 stages. The R-type instruction does not need to reference memory so it has 4 stages. When it tries to write to the same location at the same time on the write stage, hazard occurs.

5.9 Data hazards result when the register content is not written by the first instruction but is to be used by the following four instructions. The $\mathrm{rl}$ register content in the ADD instruction has not been written yet but its content is needed by the SUB, AND, and the OR instructions.

5.10 Waveform for various instructions. This waveform reflects the instructions in table 5.4.

5.11 Waveform for the LW and SW instructions. This waveform reflects the instructions in table 5.5. 


\section{CHAPTER I}

\section{INTRODUCTION}

Digital cameras have become one of the major driving forces for the future digital imaging technology. From the consumer market's perspective, digital cameras revolutionize not only the snapshot photo images but also the professional studios, including the way journalists report news and the mass media at large. Furthermore, many major United States (US) corporations have plunged into the development of digital cameras. Among them, LSI Logic developed a reduced instruction set computer (RISC) core needed to implement processing and compression capability. Major software companies like Microsoft and Adobe have developed software applications to port images from camera to personal computer (PC) and eventually to the web. Furthermore, Hewlett Packard (HP) also announced its printer for the digital cameras. Digital cameras are used to capture images into memory, which can be processed or refined for presentation clips, desktop publishing, web site usage, or multimedia presentations.

\subsection{Background}

Cameras have been one of the most powerful tools for many years in terms of capturing events and recording historical moments. In 1889, Kodak introduced the first camera that used a roll of $35-\mathrm{mm}$ film [1]. The camera was made of plastic and metal. It consisted of a mechanical aperture enclosure and film advancing mechanisms. The 
advancement in very large scale integration (VLSI) technology has been replacing many camera parts by integrated electronic circuits. Between 1960-1964, various manufacturers in the United States introduced the digital integrated circuits (ICs). Fairchild and Texas Instruments were manufacturing the resistor-transistor logic (RTL). Shortly after that, a newly formed company, Signetics, developed the diode-transistor logic (DTL) and then emerged of the transistor-transistor logic (TTL) in 1963. Also, Boyle and Smith introduced charged-coupled device (CCD) in 1970[2]. However, consumer digital cameras were not made of $C C D$ technology until very recently.

While digital technologies were developed and adopted in many applications, reliable cost-effective digital cameras have become a reality only recently. Perhaps, the first digital camera, DS-1P, was introduced by Fuji Photo Film Ltd., in 1988. It used memory card to store the images. Since then other manufacturers have also iumped onto the digital camera bandwagon. However, most of these digital cameras were overly priced for consumer marketing and they were only equipped with primitive features. For example, among them was Kodak's DCS 200 that retailed for $\$ 10,000$ and available only in a black-and-white format. Recently, compression techniques and advanced digital signal processing (DSP) technology allowed better picture qualities, higher storage capacity, and an affordable price ranges. Among others, the Casio QV-100, priced for $\$ 795$, has a color liquid crystal diode (LCD) screen for viewing the image, and is capable of storing up to 194 images depending on the mode of compression. 
Since then, other companies have manufactured cameras that deliver full motion and sound which uses joint photographic experts group (JPEG) and motion photographic experts group (MPEG) compression techniques to compress the image.

\subsection{Problem Statement}

The digital camera is in one of its most dynamic development stages. Its evolution and success depends heavily on other related technologies such as VLSI, CCD imaging, and compression standards like JPEG and MPEG.

Although we have witnessed rapid development in digital camera technology, there are many problems to be solved and questions to be answered. First of all, there seems to be lack of standardized in the development of the digital camera processors. In today's central processing unit (CPU) business, Intel's 80X86 originated Pentium Processor architecture, Motorola's alpha chip, Sun Microsystems' and SGI's MIPS processor, all targeted a specific computational need and reflect industrial acceptance. While in digital cameras, there is virtually no such architecture in existence, which poses unanswered questions for people working in the areas of bus interface, software development, and peripheral device, etc.

Secondly, the lack of evaluation of the computational need in digital cameras raises a series of question regarding the developing of processing-light vs. processingheavy capability. Equipping more computational power to digital cameras will allow the integration of many desired features by customer. However, it will also increase the cost and may complicate the usage of the camera, which could impose difficulties to the 
professional and sophisticated users. In addition, with the limited resources, more computational power usually takes the other nice features away, such as potentially less powerful interfacing control capabilities or even memories. Then, the question of how much computation power is too much, or how little computation power is too little has yet to be answered.

Last but not least, the question of what is a reasonable balance between hardware implementation and software "off-site" (meaning after down loading the images to a host computer) processing feature do we have to anticipate in order to design a user friendly, cost-effective, and powerful affordable consumer digital camera has to be addressed. This is an open question; perhaps it will take years for a true answer to come out. But we know almost for certain, that as engineers continue working on the improvement of the performance of digital cameras, this question is one that will dictate many of our design decisions, and affect the final outcome of our work.

\subsection{Objectives of This Study}

The objective of this study is to evaluate the architectural design of digital cameras, the design of the digital camera processors (DCPs), and the related hardware issues such as interface bus standards, the universal serial bus (USB), IEEE 1394, RS232, and RS400.

In particular, in this study, we would like to answer the following questions:

1. What are the architectural design characteristics of the most recently developed digital cameras? Do these characteristics reflect the general trend 
in computer central processing unit (CPU) design? Do these characteristics converge on RISC architecture?

2. What is the major computational need in digital imaging? Specifically, what is the most computationally intensive task in digital imaging? How to characterize this computation in terms of Floating-Point Operations Per Second (FLOPS)?

3. What are the common instruction sets in Digital Cameras? Which instruction set would likely be the most cost-effective solution to balance the need in computation and the cost-sensitive implementation?

4. If there were a need to develop a better processing engine and instruction set, what computational/architectural characteristics would this engine have? Can we provide simulation and verification to support the claim?

5. What is the preferable interface bus standard? How likely does the industry respond to it?

This thesis is organized in the following manner. Chapter 1 focuses on the introduction to digital cameras-the evolution of digital cameras using $\mathrm{CD}$ to capture images to using static RAM for storing images, and including a LCD display as standard feature for fast viewing of images.

Chapter 2 surveys the state-of-the-art technology in digital camera design. Digital cameras have been categorized into low-range classes, medium-range classes, and highrange classes. Also, discussion of external media such as external memory cards, the types of serial interface designs, printers for digital cameras, and software applications 
for digital cameras is provided. Furthermore, the general architectural design and implementations, the digital signal processing capability, and the I/O interface design were the focus in the survey.

Chapter 3 analyzes the digital camera's architectural design. It describes how the RISC core processor, combined with the DSP core processor, served as a powerful lowpower consumption device that is used in digital camera applications. Also, analyzed is how the instruction set architecture and the arithmetic logic unit (ALU) are integrated to achieve processing power for image compression. Furthermore, the types of buses and I/O controller designs between major processors are discussed. Finally, the benchmarks of the performance for these processors are the focus of the last part for chapter 3.

Chapter 4 suggests an architectural design for high-speed, high-performance digital camera applications. The performance is focused on the general computational capability, the potential for a big family of signal processing functions, faster I/O interface, and better cost-performance memory unit. Also, an evaluation of the need to support modern operating systems is presented.

Chapter 5 is the hardware description language (HDL) simulation of the RISC architecture. It is a 16-bit MIPS instruction set architecture. It has 5 stage pipelines with LOAD/STORE implementation.

Chapter 6 is the conclusion of this thesis. It highlights the evolution of digital cameras, summarizes the state-of-the-art digital cameras, and suggests architectural design for the digital camera applications. 


\section{CHAPTER II}

\section{LITERATURE SURVEY}

Many companies are competing to produce a better digital camera in the most cost-effective manner. This chapter surveys the state-of-the-art technology in digital cameras. First, we focus on the high-end digital cameras available in the consumer market. From the high-end digital cameras, we identify the key features that were implemented on these cameras. Then, we identify the external media such as external memory cards, $/ / O$ interface to computer and printer, and software applications.

\subsection{The State-of-the-Art Technology in Digital Camera Applications}

Digital cameras are in a dynamic stage of development. Today's state-of-the-art digital cameras may not remain state-of-the-art by tomorrow. It is difficult to measure the state-of-the-art digital camera when several new cameras are introduced by various manufacturers every month. However, it is our intention to look into the core technology that enables this fast paced development and categorize digital cameras into three major classes based on price range, which reflect the overall features and quality of the cameras. Given in table 2.1 is the guideline for price range breakdown. Note that the high-end cameras well over $\$ 10,000$ range are for professional imaging applications. Table 2.2 shows an example of the features for various high-range cameras by different manufacturers. Key features in high-range cameras are the higher image resolution, higher color-depth, bigger memory storage space, and more user friendly features. 


\begin{tabular}{|l|l|}
\hline Category & Price Range \\
\hline Low-range & $\$ 170.00-\$ 1,000.00$ \\
\hline Middle-range & $\$ 1,001.00-\$ 10,000.00$ \\
\hline High-range & $\$ 10,001.00-\$ 60,000.00$ \\
\hline
\end{tabular}

Table 2.1: Category of digital camera by price.

\begin{tabular}{|c|c|c|c|c|c|}
\hline Features & $\begin{array}{c}\text { Eastman } \\
\text { Kodak } \\
\text { DCS-460, } \\
\text { DCS-465 }\end{array}$ & $\begin{array}{c}\text { Scan View } \\
\text { Inc. } \\
\text { Carnival } \\
2000 \text { s }\end{array}$ & $\begin{array}{c}\text { Sony } \\
\text { DKC-ST5 }\end{array}$ & $\begin{array}{c}\text { Sinar Bron } \\
\text { SinnarCam }\end{array}$ & $\begin{array}{c}\text { Dicomed } \\
\text { Inc. } \\
\text { BigShot } \\
4000\end{array}$ \\
\hline $\begin{array}{c}\text { Pixel } \\
\text { Resolution }\end{array}$ & $3060 \times 2036$ & $2048 \times 2048$ & $2560 \times 2048$ & $2048 \times 2048$ & $4096 \times 4096$ \\
\hline $\begin{array}{c}\text { Exposure } \\
\text { (shots) }\end{array}$ & 1 & 1 or 4 & 1 & 1 or 3 & 1 \\
\hline $\begin{array}{c}\text { Color Depth } \\
\text { (bits) }\end{array}$ & 36 & 36 & 30 & 42 & 36 \\
\hline Interface & SCSI & SCSI & SCSI & PCI & SCSI \\
\hline $\begin{array}{c}\text { Inter RAM } \\
\text { (MB) }\end{array}$ & 18 & 22.8 & 15 & 24 & 160 \\
\hline Mac/PC & Y $/ \mathrm{Y}$ & Y/N & Y/Y & Y/N & Y/Y \\
\hline Price & $\$ 27,500$ & $\$ 29,995$ & $\$ 31,500$ & $\$ 43,500$ & $\$ 55,900$ \\
\hline
\end{tabular}

Table 2.2: List of high-range digital camera with some key features. 


\subsection{Digital Camera System}

Figure 2.1 shows a block diagram of a typical digital camera system. Functionally, it consists of the imager block, signal processing and system block, and external media block. The imager block has the CCD unit, white balance, and the flash. The signal processing and system block consists of the compression and decompression (CODEC) unit, color spacing, CPU, and local memory. Finally, the external media block consists of the serial input/output (I/O) unit, national television systems committee/phase alternating lines (NTSC/PAL) controller unit, personal computer memory card international association (PCMCIA) controller unit, and the LCD controller unit.

The CCD unit includes the sensor to capture the image from an optical lens and an analog to digital (AD) converter. The $C C D$ is a solid-state chip containing lightsensitive photocells that are arranged into columns and rows. Shown in figure 2.2 is a typical CCD diagram. The CCD has an optical black area, active pixel area, and effective pixel area. The effective pixel area is where the image will actually be captured. The horizontal shift registers store the value of each column of photocells. The photocells convert light into electrons to record a three-dimension (3D) scene in color. A higher color-depth allows a pixel to be represented in a broader dynamic range (the term colordepth and pixel-depth is used interchangeably). Most of the high-end cameras in Table 2.2 have at least 36-bit color-depth, which is capable of displaying 4,096 colors in each of the RGB color for a total of 12,288 different colors.

It is determined that any color can be produced by a combination of red, green and blue (RGB). Therefore, RGB filters can be placed in front of the sensor to capture 
color images. Color filter arrays (CFA) come in several types. RGB filter places each filter in front of the sensor, one filter for each RGB color. This type of CCD requires three exposures or shots, one for each color. Thus, an ideal application for this CCD technology is limited to only studio or still photo. Yet, another method to get CCD color is to place filters to capture the chrominance and another to capture the luminance. This type of CFA requires two CCD sensors, one CCD to capture the luminance and the other CCD to capture the chrominance. Digital cameras that use this type of two-CCD technology are referred to as two-chip cameras. A third type of CFA is to place a RGB filter over the pixel. This method requires one-CCD sensor to capture all three colors in a single exposure. Figure 2.2 shows a diagram of the one-CCD sensor. Figure 2.3 shows the arrangement of the RGB filters to capture color image according to a pattern known as the Bayer pattern [3]. The Bayer pattern is mostly used in current CCD design for still digital cameras. However, other pattern arrangements such as the HP pattern also claim to have better image recognition than the Bayer pattern. One of the most recently developed CCD that uses this one-CCD technology is the M6 by Eastman Kodak, which is able to capture up to 6.3 million pixels [35]. The M6 CCD is used for the Kodak's professional DCS-460 and DCS-465 digital cameras that are priced at $\$ 27,500$.

Future CCD technology will be in the hundredths of Mega-pixel. [4-6] discussed many techniques on improvements for current CCD technology. Philips has developed a 63 million pixel CCD with 12 micron pixel sizes and plans for a one Giga-pixel CCD is underway. Also, Loral is currently making an 81 million pixel $C C D$ on a 5-inch silicon wafer [4]. Presently, these CCD technologies are not targeted for consumer electronics. 
They are targeted for scientific research cameras. However, it is clear that eventually high-range cameras will be incorporating these high-resolution CCD designs into digital cameras in the near future.

The color spacing unit takes the RGB sample and converts it into $\mathrm{YCrCb}$ color coordinate according to equation 2.3a-2.3d in section 2.2.3.1. This unit also prepares the $\mathrm{YCrCb}$ in the form of matrix for the CODEC unit. The CODEC unit performs compression and decompression on the $\mathrm{YCrCb}$, which represents the image. The $\mathrm{CPU}$ coordinates the operation of each unit and controls the user interface. Such user interface includes programming features such as manual selection of flash level, black and white balance level, and others. 


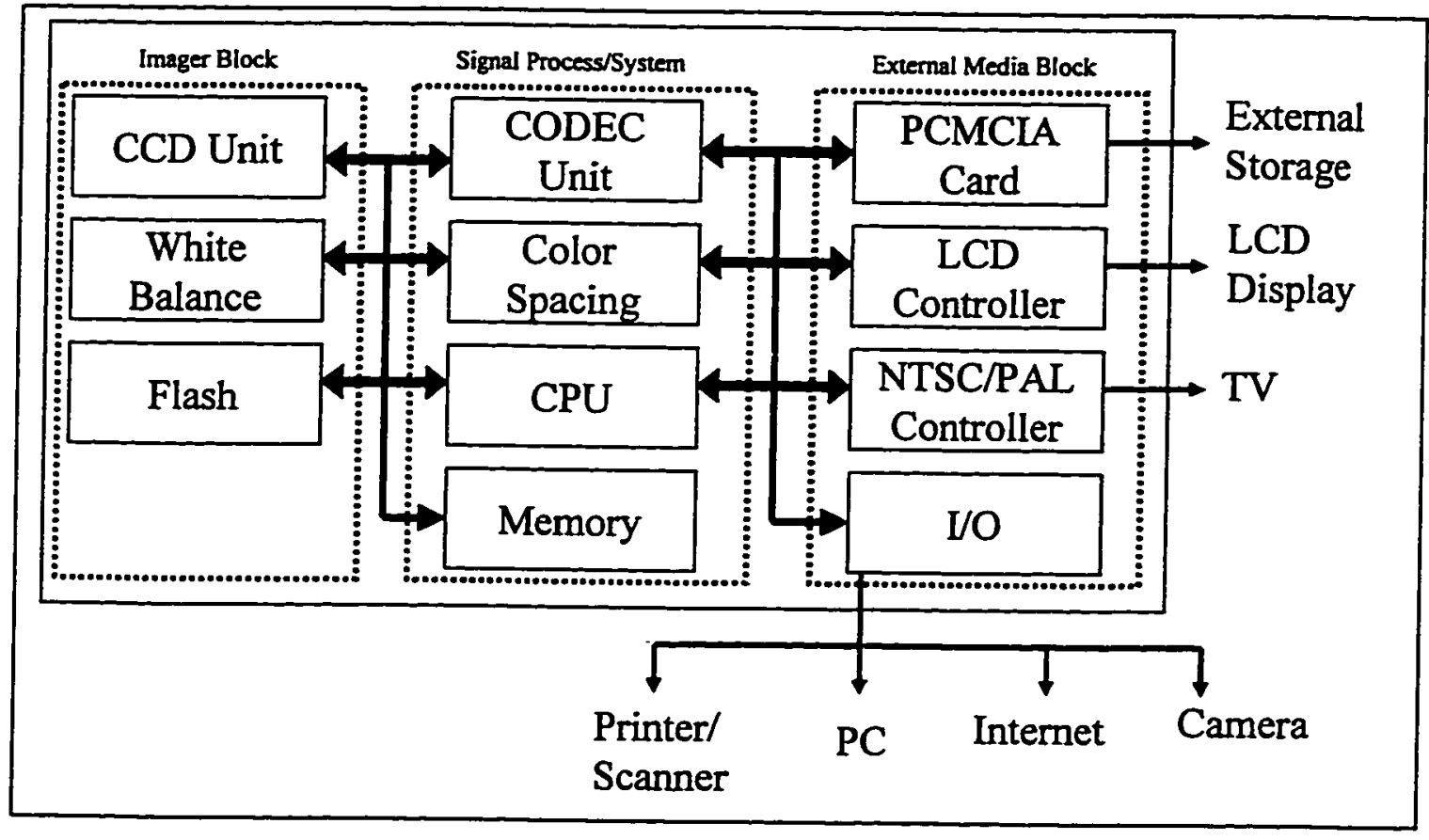

Figure 2.1: The major blocks of a typical digital camera. The overall blocks are classified into imager block, signal processor/system block, and external media block. 


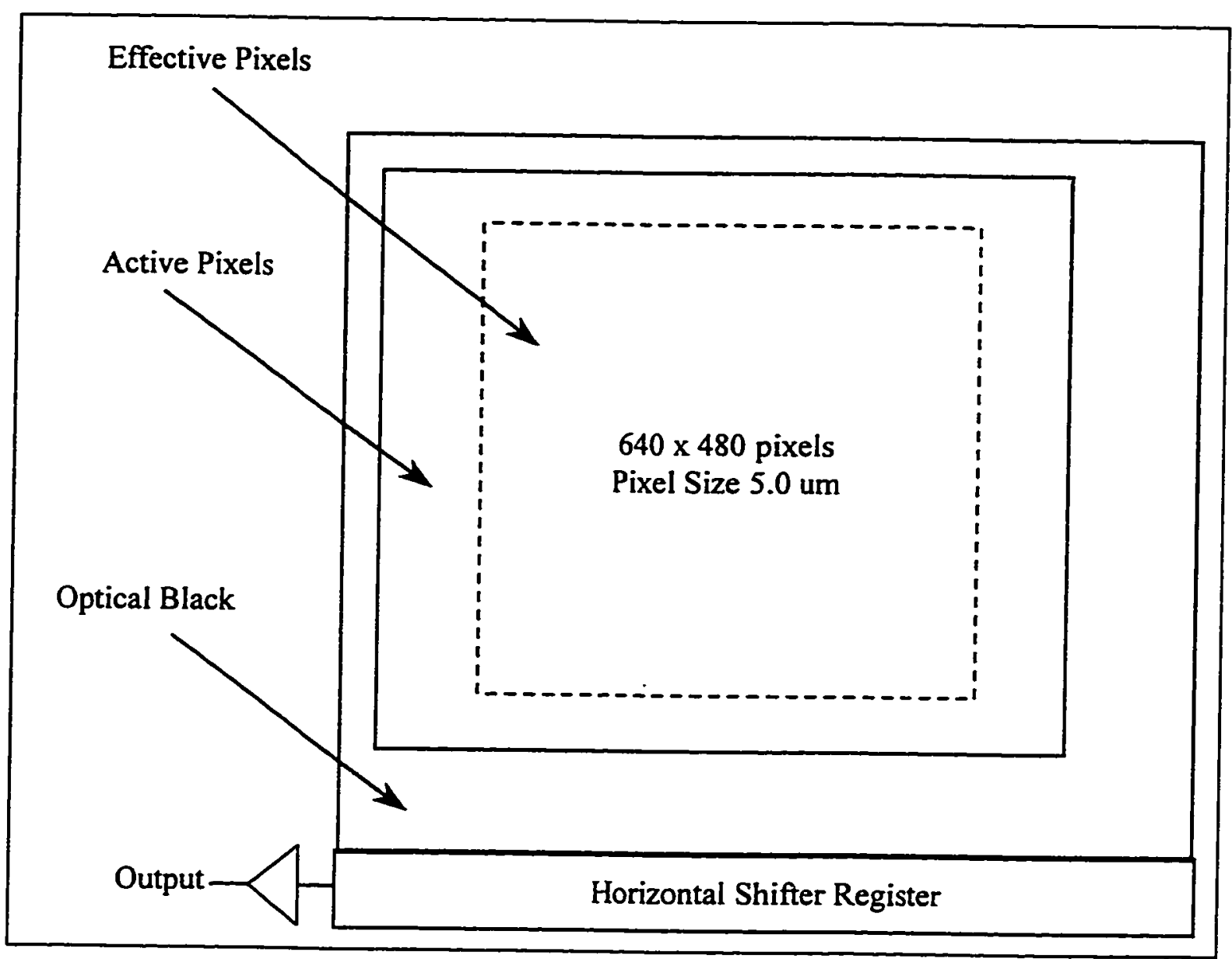

Figure 2.2: A typical diagram of CCD design [34]. The optical black is used to reference how much darkness is applied during the capturing process. Both the active pixel area and the effective pixel area are used to store the image. However, only the effective pixel area is valid for display. 


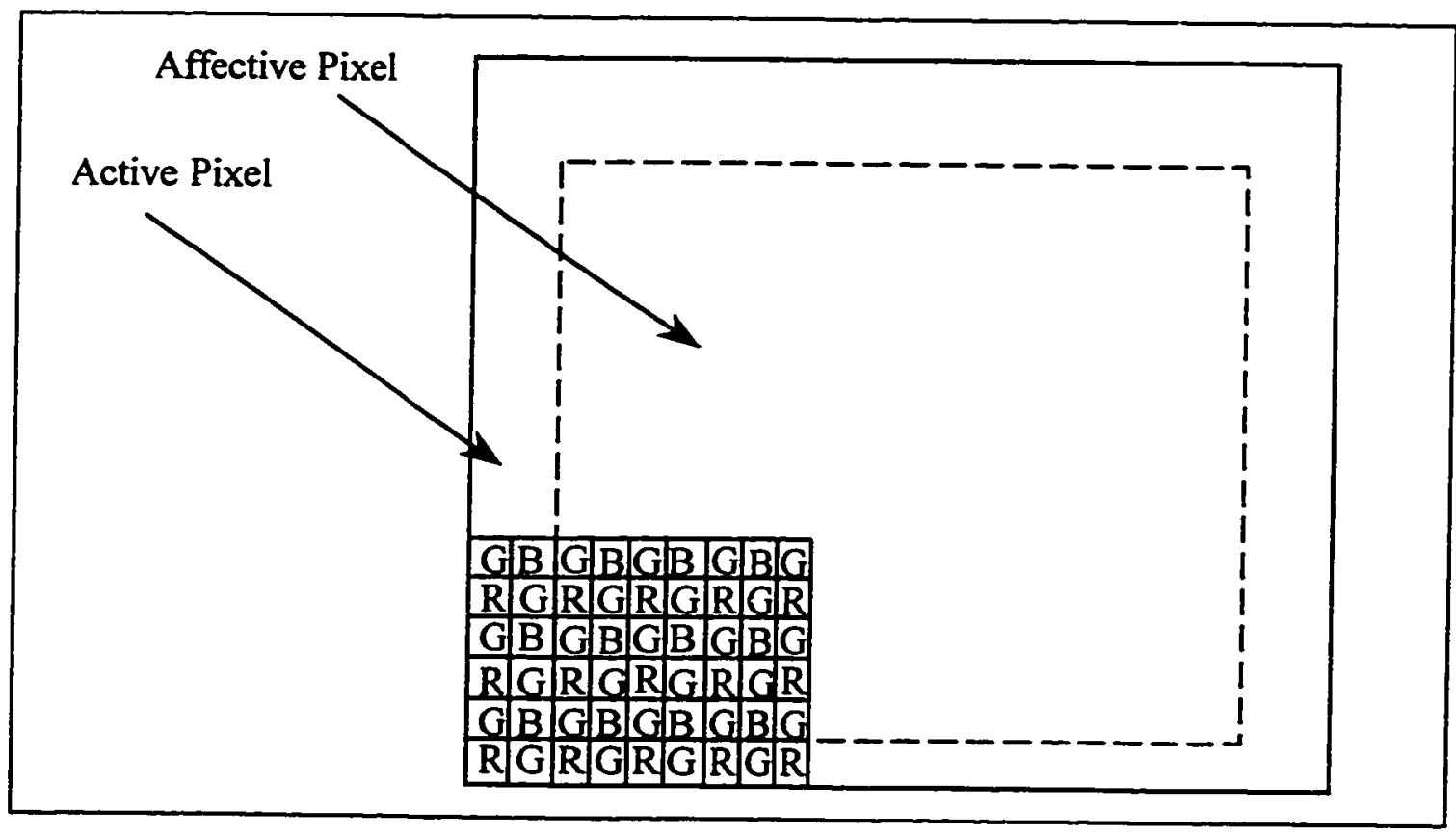

Figure 2.3: A Bayer pattern arrangement of RGB color for one-exposure CCD. Both the active pixel area and the affective pixel are arranged with the Bayer pattern. 


\subsubsection{Capture Mode}

In capture mode, the camera goes through series of steps as shown in figure 2.4. First, the image is captured by the CCD through the camera lens. The A/D converter converts the analog representation of the image into digital representation. The digital image is buffered in the dynamic read access memory (DRAM) ready to be processed by the color space unit. The color space unit converts the image from RGB to $\mathrm{YCrCb}$ format. The output of the color space unit feed into the CODEC unit for compression. The compressed image goes through the DRAM buffer and is stored into an external memory device.

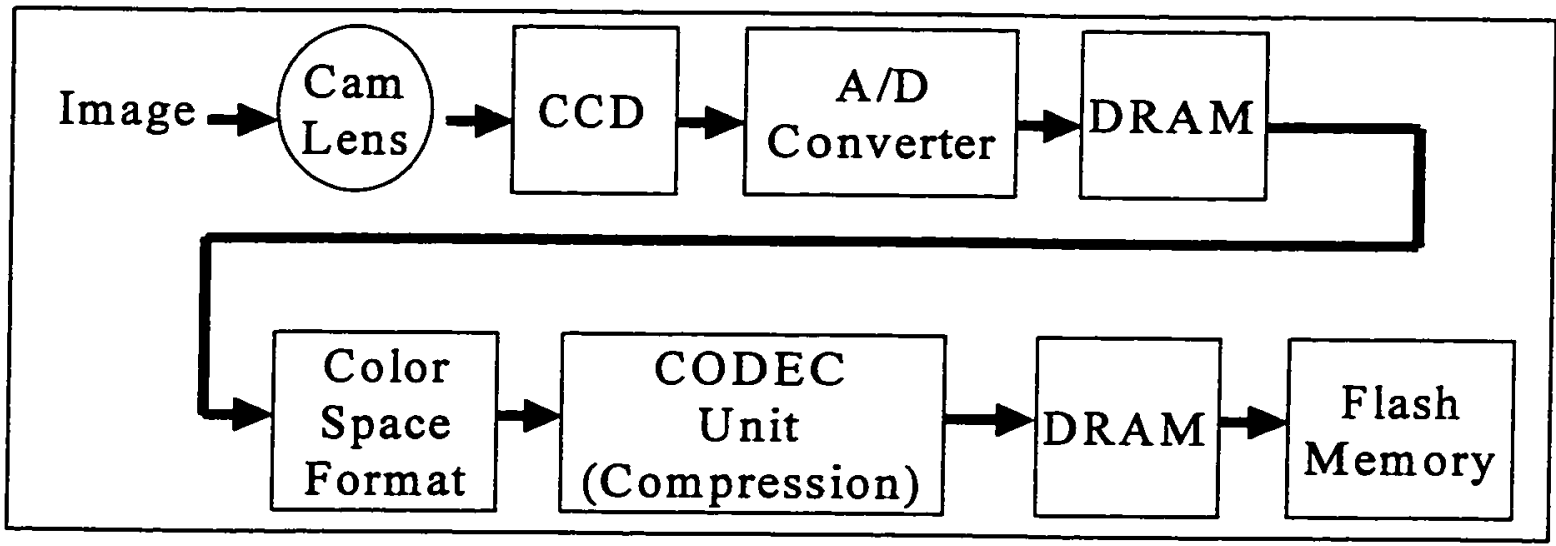

Figure 2.4: The capturing sequence of an image. 


\subsubsection{Display Mode}

In the display mode, the compressed image is retrieved from the flash memory into the DRAM buffer. The CODEC unit reads the image from the DRAM buffer and performs decompression. The color space unit converts the image into an appropriate format for display. Then the image sample goes through another DRAM buffer. The display controller reads the image sample from the DRAM buffer and displays it on the LCD screen.

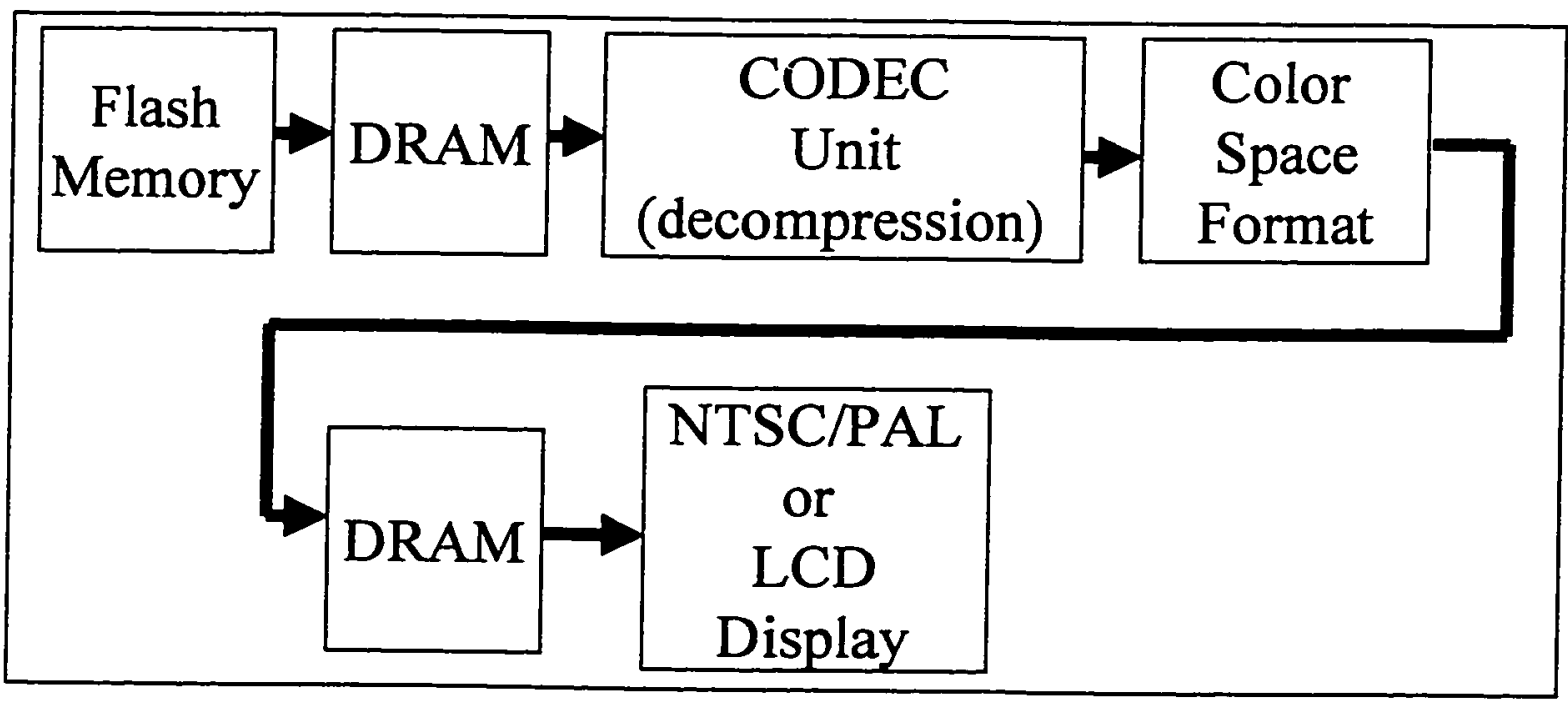

Figure 2.5: The displaying sequence of a digital camera. 


\subsubsection{Digital Camera CODEC}

One of the major break throughs in digital camera design is the CODEC unit. The process of CODEC implementation has been an evolution from mathematical formulation to VLSI implementation. Figure 2.6 shows the major block diagram of an encoding scheme used in digital cameras today. Currently, the majority of consumer digital cameras uses JPEG standards.

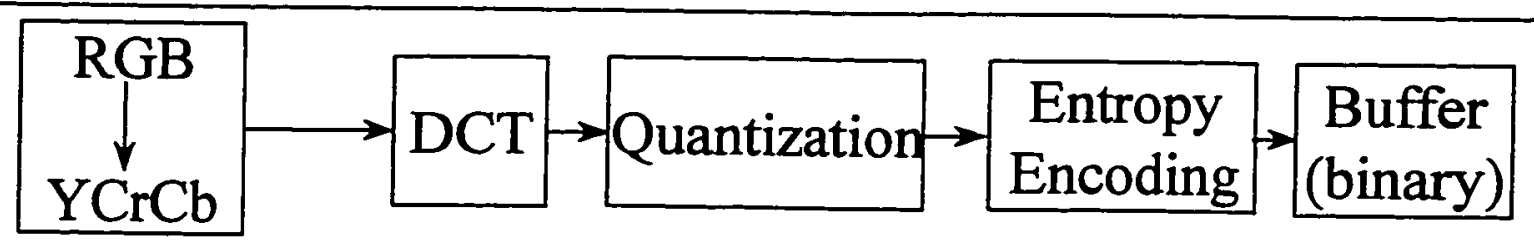

Figure 2.6: Major blocks of JPEG encoding standard.

\subsubsection{JPEG Standard}

The JPEG standard starts by converting image format from RGB to $\mathrm{YCrCb}$. Many digital cameras are designed to be NTSC or PAL compatible. This leads to converting the RGB format to YUV format which is used in European TV or to the YIQ format used in North American TV. The YUV format is obtained from the RGB in the following way for linear color transformation [9]: 
(2.1a) $Y=0.3 * R+0.6 * G+0.1 * B$

(2.1b) $V=R-Y$

(2.Ic) $U=B-Y$

The $\mathrm{Y}$ component is known as the luminance. The $\mathrm{V}$ and $\mathrm{U}$ are known as the chrominance components. The YIQ format is related to YUV in the following ways [9]:

(2.2a) $\mathrm{I}=0.74 * \mathrm{~V}-0.27 * \mathrm{U}$

(2.2b) $\mathrm{Q}=0.48 * \mathrm{~V}+0.41 * \mathrm{U}$

The $\mathrm{Y}$ in YIQ is the same $\mathrm{Y}$ as in the YUV color format. The change in the YIQ is the I and Q component. Furthermore, another color system besides the YUV or YIQ is the $\mathrm{YCrCb}$ color format used for JPEG standard. It has the following relationship with the YUV format [9].

(2.3a) $\mathrm{Cb}=\mathrm{U} / 2+0.5$

(2.3b) $\mathrm{Cr}=\mathrm{V} / 1.6+0.5$

The $\mathrm{YCrCb}$ can be computed directly from the RGB format or the RGB can be computed from $\mathrm{YCrCb}$ via the following equation [33]:

$(2.3 c) \quad\left[\begin{array}{l}Y \\ C r \\ C b\end{array}\right]=\left[\begin{array}{ccc}0.299 & 0.587 & 0.114 \\ 0.500 & -0.419 & -0.081 \\ -0.169 & -0.331 & 0.500\end{array}\right]\left[\begin{array}{l}R \\ G \\ B\end{array}\right]$ 


$$
(2.3 d) \quad\left[\begin{array}{l}
R \\
G \\
B
\end{array}\right]=\left[\begin{array}{ccc}
1.0 & 1.402 & 0 \\
1.0 & -0.419 & -0.3437 \\
1.0 & -0.331 & 1.772
\end{array}\right]\left[\begin{array}{c}
Y \\
C r \\
C b
\end{array}\right]
$$

The $\mathrm{YCrCb}$ output is fed into the DCT unit. The DCT unit performs fast discrete cosine transform (DCT). Some digital cameras realize the DCT computation in software while others realize it in hardware. The implementation of two dimension (2-D) DCT in JPEG standard is based on each individual 8 X8 macroblock. The forward and backward 2-D DCT transform is defined as follow [7]:

$$
\begin{gathered}
X(k, l)=\frac{c(k) c(l)}{4} \sum_{i=0}^{7} \sum_{j=0}^{7} x(i, j) \operatorname{co}\left(\frac{(2 i+1) k \pi}{16}\right) \cos \left(\frac{(2 j+1) k \pi}{16}\right) \\
c(k), c(l)= \begin{cases}\frac{1}{\sqrt{2}} & \text { if } \quad k=0 \\
1 & \text { otherwise }\end{cases}
\end{gathered}
$$

where $k, l=0,1, \ldots, 7$

$$
x(i, j)=\sum_{k=0}^{7} \sum_{l=0}^{7} \frac{c(k) c(l)}{4} X(k, l) \cos \left(\frac{(2 i+1) k \pi}{16}\right) \cos \left(\frac{(2 j+1) k \pi}{16}\right)
$$

Next, the quantization unit performs further information reduction by representing a given image with limited quantization levels and by removal of high frequency components. The design of the quantization unit is based on the studies of human visual 
perception, which allows the reduction of image details with or without very little noticeable visual artifacts. Based on the characteristics of human visual perception study, JPEG standard uses the following quantization matrices as shown in Tables 2.3 and 2.4.

$\begin{array}{llllllll}16 & 11 & 10 & 16 & 24 & 40 & 51 & 61 \\ 12 & 12 & 14 & 19 & 26 & 58 & 60 & 55 \\ 14 & 13 & 16 & 247 & 40 & 57 & 69 & 56 \\ 14 & 17 & 22 & 29 & 51 & 87 & 80 & 62 \\ 18 & 22 & 37 & 56 & 68 & 109 & 103 & 77 \\ 24 & 35 & 55 & 64 & 81 & 104 & 113 & 92 \\ 49 & 64 & 78 & 87 & 103 & 121 & 120 & 101 \\ 72 & 92 & 95 & 98 & 112 & 100 & 103 & 99\end{array}$

Table 2.3: The luninance quantization table [9].

$\begin{array}{llllllll}17 & 18 & 24 & 47 & 99 & 99 & 99 & 99 \\ 18 & 21 & 26 & 66 & 99 & 99 & 99 & 99 \\ 24 & 26 & 56 & 99 & 99 & 99 & 99 & 99 \\ 47 & 66 & 99 & 99 & 99 & 99 & 99 & 99 \\ 99 & 99 & 99 & 99 & 99 & 99 & 99 & 99 \\ 99 & 99 & 99 & 99 & 99 & 99 & 99 & 99 \\ 99 & 99 & 99 & 99 & 99 & 99 & 99 & 99 \\ 99 & 99 & 99 & 99 & 99 & 99 & 99 & 99\end{array}$

Table 2.4: The chrominance quantization table [9]. 
Entropy encoding is the last block in the JPEG standard, which encodes the quantized image by either Huffman coding or arithmetic coding. In a baseline implementation, a look-up table technique for Huffman coding is provided. The arithmetic coding is based on the notion of mapping each pixel intensity to an interval between 0 and 1. After the entropy encoding the image may go through another coding stage, beyond the JPEG standard encoding, called channel coding to prepare for transmission. Depending on the type of transmission cable used, the channel coding may include bit-stuffing, cycle-redundancy-check (CRC) or other coding schemes before the image data goes through the cable.

\subsubsection{CODEC IC}

Early implementation of the compression technique takes several IC chips. Table 2.5 shows some of the commercially available ICs for image and video CODEC. An example of multiple chip implementation is the LSI Logic's LS647xx series. The L64765 implements the color space which preprocesses the pixel $\mathrm{YCrCb}$ and matrix vector for DCT transform. The L64735 does the DCT transform. The L64745 does the JPEG entropy coding. Some latest IC can implement the JPEG CODEC in one or two chips. Currently, most digital cameras implement the CODEC in two or more chips. Along with the I/O IC and CPU for controlling, a digital camera is implemented in as many as seven chips. 


\begin{tabular}{|l|c|c|c|c|}
\hline Vendor & Part Number: & Standard & Encoder & Decoder \\
\hline C-Cube Microsystems & CL450 & JPEG & & $\sqrt{ }$ \\
\hline LSI Logic & LS647xx & JPEG & $\sqrt{ }$ & $\sqrt{ }$ \\
\hline AT\&T & AVP-1300E & JPEG & $\sqrt{ }$ \\
\hline & AVP-1400D & JPEG & & $\sqrt{ }$ \\
\hline $\begin{array}{l}\text { Integrated Information } \\
\text { Technology }\end{array}$ & VP & JPEG & $\sqrt{ }$ \\
\hline SGS-Thompson & STV3208 & JPEG & & \\
\hline
\end{tabular}

Table 2.5: Commercially available ICs for JPEG.

\subsection{Digital Camera External Accessories}

This section deals with external interfaces such as external memory, battery types, power supplies, types of serial interfaces, printers for digital cameras and software applications.

\subsubsection{Memory Cards}

Some cameras have limited internal memory to store the images. External memory cards were designed to expand the memory through PCMCIA card, Flash card, Smart Media Memory card, or other proprietary memory card. The PCMCIA has type I, 
II, and III. However, type I is quite thin and therefore contains less memory storage. Thus, mainly type II and III are used as shown in table 2.6.

\begin{tabular}{|c|c|l|c|}
\cline { 2 - 4 } \multicolumn{1}{c|}{} & $\begin{array}{c}\text { Available } \\
\text { Size (MB) }\end{array}$ & \multicolumn{1}{|c|}{$\begin{array}{c}\text { Camera Employing } \\
\text { This Type of Card }\end{array}$} & $\begin{array}{c}\text { Price } \\
\text { (US Dollars) }\end{array}$ \\
\hline Type II & 4 & $\begin{array}{l}\text { Kodak DC-50; } \\
\text { Ricoh (RDC-1, RDC-2, RDC-2E); } \\
\text { Canon PowerShot 600; } \\
\text { Minolta RD-175 and others }\end{array}$ & $\$ 112.00$ \\
\cline { 2 - 4 } & 64 & $\begin{array}{l}\text { Hitachi MP-EG1A; } \\
\text { Canon PowerShot 600; } \\
\text { Minolta RD-175 and others }\end{array}$ & $\$ 751.00$ \\
\cline { 2 - 5 } & 170 III & 340 &
\end{tabular}

Table 2.6: PCMCIA Memory Cards available for various digital cameras.

On the other hand, flash cards range from $2 \mathrm{MB}$ to $20 \mathrm{MB}$. The price for a flash card ranges from $\$ 79.99$ for a $2 \mathrm{MB}$ to $\$ 325.00$ for a $20 \mathrm{MB}$. Flash cards fit Kodak (DC210, DC-120, and DC-25), Panasonic (KXL-600a and 601a), Canon PowerShot 350, and Epson PhotoPC 600.

Yet, smart media memory card fits the following cameras: Minolta Dimage V, and Olympus (D-220L, D-320, D-500, and D-600). Currently, smart media memory cards are available for $4 \mathrm{MB}$ at $\$ 57.95$ per card.

Lastly, proprietary memory cards are produced by various manufacturers. Vivitar Vivicam 3000 has 4 MB memory card at $\$ 99.99$ per card. Epson PhotoPC 500 has 4 MB memory card for $\$ 245.00$ per card and 2 MB for $\$ 145.00$. 


\subsubsection{I/O Interface}

Currently digital camera $\mathrm{L} / \mathrm{O}$ interface is mainly based on a RS-232 serial communication. A few have implemented the USB serial interface. This interface is used mainly for cameras that have direct connections to a PC. Therefore, power to the camera is supplied by the USB connection and no battery operation is necessary if connected to a PC. Yet, others have implemented the wireless infrared transmission. The infrared communication is between the camera and external device like a printer.

\subsubsection{Software Applications}

Since the development of digital cameras, manufacturers have launched their own software application along with their digital cameras to allow further image processing on a PC. However, this causes confusion to the users in that there are too many formats and is incompatible for desktop publishing and internet applications. Recently, Eastman Kodak, Hewlett-Packard Company, Live Picture Inc., and Microsoft developed FlashPix, an image file format and digital image manipulation. FlashPix is intended to become an international standard. FlashPix specifies a hierarchical digital representation of the image in thumbnail, and viewing parameters. Also, other features included are file links, image manipulation, and application-specific data. For example, a printer automatically prints the high resolution image in the hierarchy where an internet link views only the thumbnail resolution [36]. 


\section{CHAPTER III}

\section{EVALUATION OF THE ARCHITECTURAL DESIGN}

In this chapter, we turn our focus into the evaluation of the newest commercially available general-purpose architectural design that could be used to implement digital cameras. To evaluate the architectural design for digital camera applications, we start this chapter on the architectural design of several general-purpose processors. Next, we will look at the instruction set design, the I/O interface design and the benchmarks of the performance for the architectural designs.

\subsection{The Architectural Design}

There are several criteria in choosing a general-purpose architectural design for digital camera applications. First, the architectural design should support the implementation of JPEG standard for image compression and decompression. Second, the architecture ought to support high-speed I/O interface for porting data to a PC, printer, scanner, or an external storage device. Third, the architecture should exhibit a low-power consumption feature, which ideally can be implemented most effectively in a single chip rather than multiple chips. Fourth, an important criteria for image processing, the architecture must be able to process an image in realtime. 
In this section, we discuss several newly available general-purpose embedded architectural designs that contain features satisfying the criteria described above. These architectural designs were the results of combining DSP processor and RISC processor to provide all the functions needed to implement low-cost yet relatively powerful processing power for digital cameras.

\subsubsection{Matsushita Electric Industrial's VDSP2 Architectural Design}

Like many semiconductor companies, Matsushita Electric Industrial focuses on designing a microprocessor for multimedia applications. The VDSP2 is the second generation of video signal processor from Matsushita Electric Industrial. The VDSP2 is intended for MPEG implementation. The VDSP2 has four individual programmable vector processors (VPs), VP0-VP3. Each VP has its own ALU, a 24-bit Adder, a 16x16bit multiplier accumulator (MAC), and five memory blocks: data block memory (DBM), source block memory (SBM), reference block memory (RBM), working block memory (WBM), and code block memory (CBM). In addition, the VDSP2 is equipped with a RISC processor that serves as the controller. The RISC processor has instruction cache, data cache, an ALU, a barrel shifter, and a 16x16-bit multiplier. Also, VDSP2 includes a hardwared DCT/IDCT processor that can execute $8 \times 8$ square pixel array effectively.

Lastly, VDSP2 has variable length coding (VLC) and variable length decoding (VLD) circuitry for Huffman encoding and decoding. The VLC/VLD processor consists of a RISC type controller for table look up in implementing its variable length encoding 
and decoding circuitry [25-26].

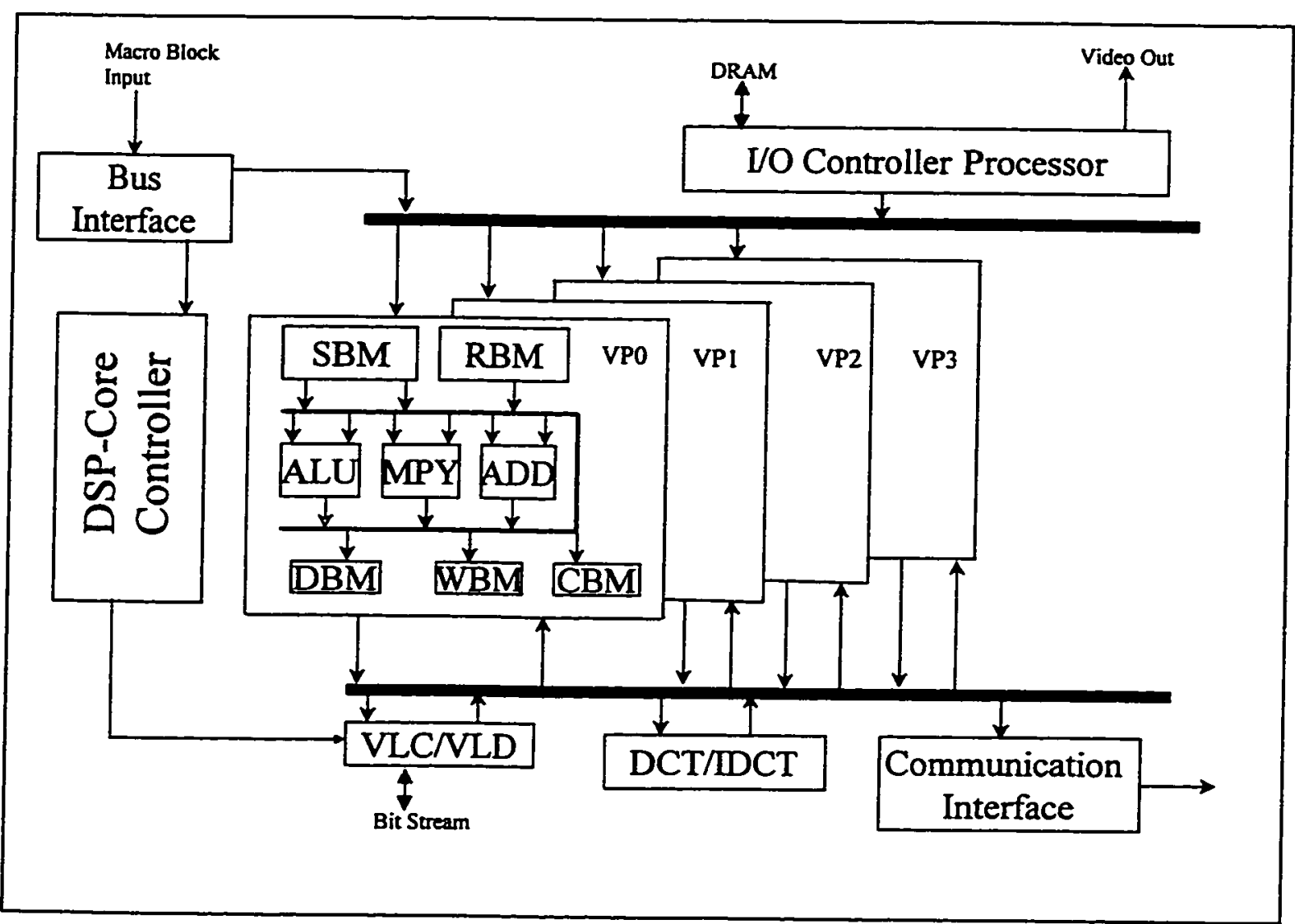

Figure 3.1: Block diagram of the Matsushita Electric Industrial's VDSP2 processor. 


\subsubsection{Hitachi's SH-DSP HD643710F Architectural Design}

The SH-DSP HD643710F is a RISC processor with the extended DSP processing capability by Hitachi [29]. It does not have hardware CODEC circuitry. It performs the CODEC functions via software. However, its on-chip peripheral module includes a serial communication interface (SCD), serial debug interface (SDI) and system controller (SYSC) that provides the system control capability for digital camera application.

\subsubsection{SH-DSP CPU}

The SH-DSP HD643710F's CPU contains a fetch unit, decode unit, DSP unit, an integer unit and register files. The fetch unit primarily fetches instruction from memory through the I-bus, X-bus, or the Y-bus. The fetched instruction is then decoded in the decoding unit and fed to the integer unit and the DSP unit for further processing. The DSP unit uses five-stage pipelines system with both von Neumann architecture and the extended Harvard architecture. The standard von Neumann architecture shares the same bus for both data and instruction. As for the extended Harvard architecture, instruction and data have separate buses to allow simultaneous access to memory. Furthermore, the DSP performs a 16x16-bit multiplication with the result of 64-bit in one cycle.

On the other hand, the integer unit primarily performs ALU related operations. It

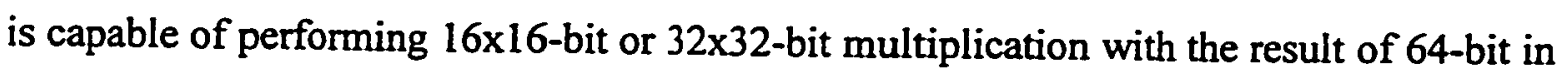
one to four clock cycles. Also, it has a built-in MAC function capable of performing $16 \times 16+64-$ bit or $32 \times 32+64-$ bit operation with the result of 64-bit in two to four clock 
cycle.

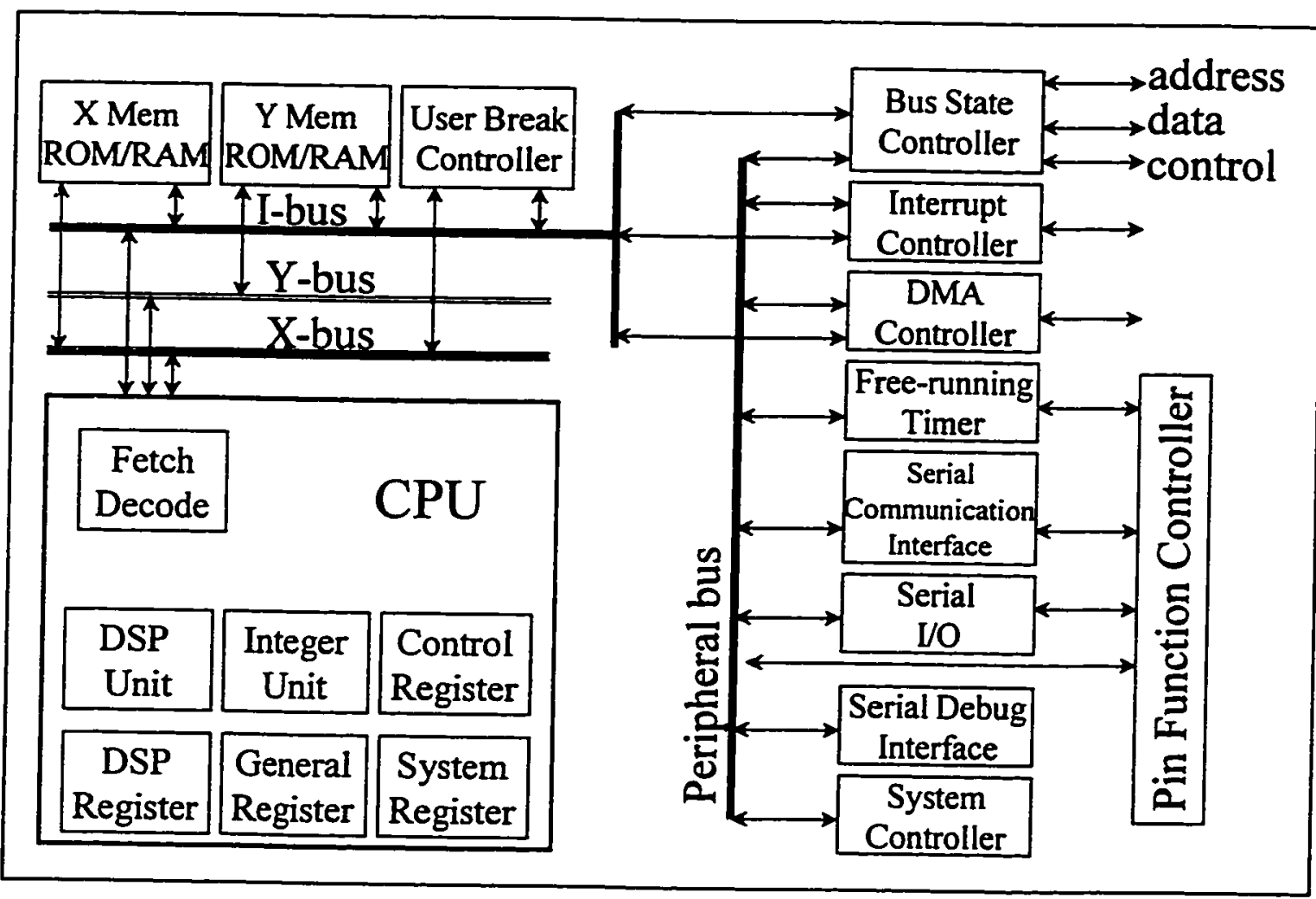

Figure 3.2: Block diagram of the Hitachi's SH-DSP HD643710F processor [29]. 


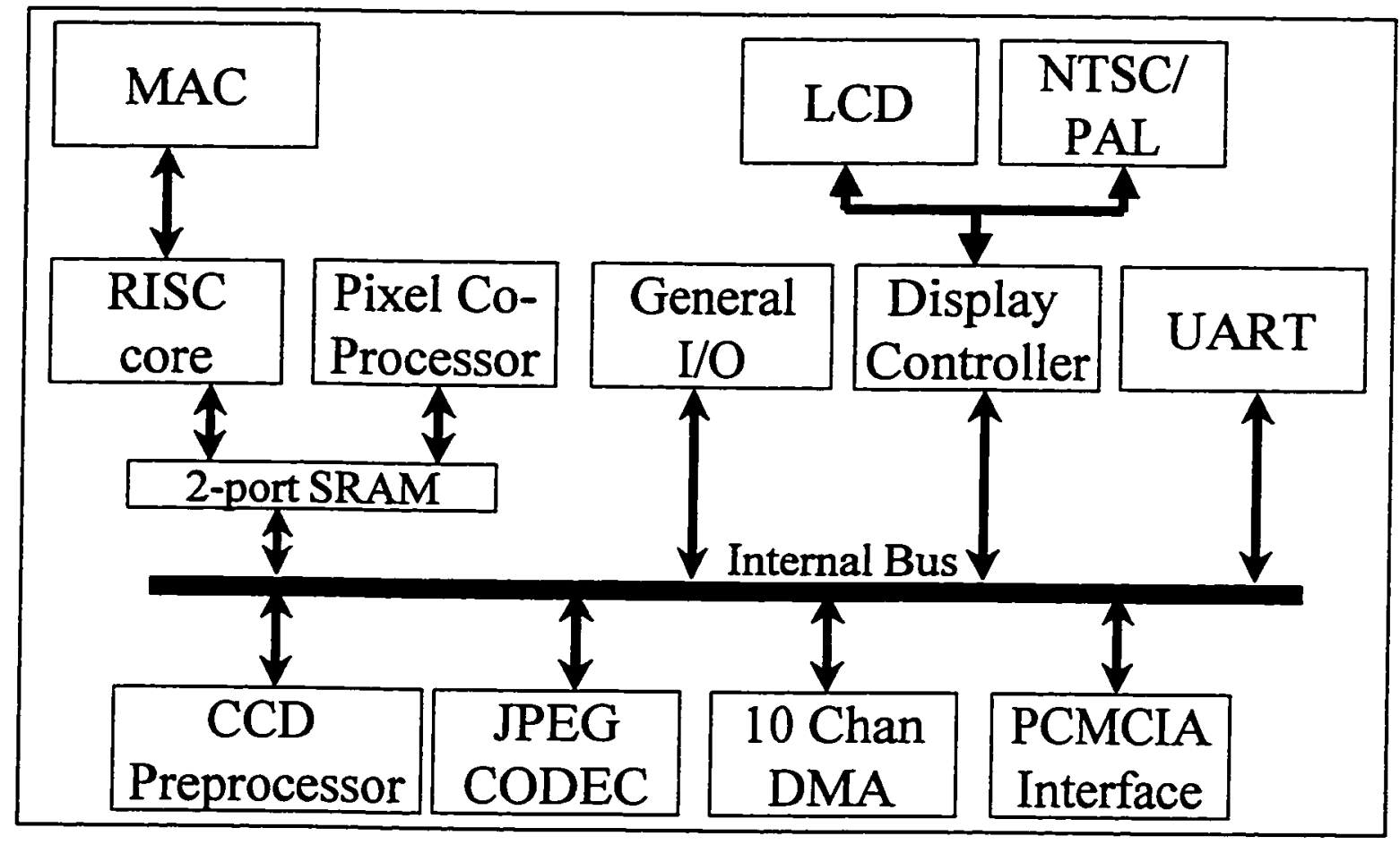

Figure 3.3: Block diagram of the LSI Logic's DCAM-101 processor [30]. 


\subsubsection{LSI Logic's DCAM 101 Architectural Design}

On May 19, 1997, LSI Logic announced the DCAM-101 chip that shrinks all major electronic components relevant to digital camera applications into one-single chip. The DCAM-101 was designed in conjunction with LSI Logic and Minolta corporations. The major components of the DCAM-101 includes a processor unit, an interface unit, a JPEG CODEC unit, a clock/power management unit, a DMA controller unit and a miscellaneous function unit all interconnected through an internal bus [30].

\subsubsection{DCAM-101 Processor Unit}

The processor unit includes the RISC core, instruction cache, data cache, two-port SRAM, a pixel coprocessor, and the MAC. The RISC core is derivative of the MIPS $\mathrm{R} 3000$ architecture that operates at $54 \mathrm{MHz}$. It runs the MIPS II instruction set. The RISC core improved its performance by exploiting direct-mapping memories. These include the instruction cache and the data cache. The instruction cache is a 2-KB cache and the data cache is a $256-\mathrm{KB}$ cache. In addition, the 2-KB SRAM implements as a two-port buffer between the processor and the internal bus. This is the interface port between the processor unit and the major components in the DCAM-101. Furthermore, the pixel coprocessor is another element in the processor unit. The pixel coprocessor performs edge enhancement, color space conversion, and pixel interpolation. In addition to these main functions, the pixel coprocessor also performs image resizing. Lastly, the 
MAC is used mainly for specific user programmable image processing. This is because matrix filtering could be done in the pixel coprocessor or image CODEC could be done in the JPEG unit. Thus, this MAC serves as a user programmable image processor.

\subsubsection{DCAM-101 JPEG CODEC Unit}

The JPEG CODEC unit implements the JPEG standard for image compression and decompression in hardware. This includes Huffman coding and decoding, quantization, and DCT algorithm.

\subsubsection{Motorola's MPC823 Architectural Design}

On May 6, 1996, Motorola added the MPC823 to their embedded family of MPC82x processor. The MPC821 and MPC823 microprocessor with DSP functionality are capable of performing JPEG or MPEG1 CODEC. The MPC823 has several interesting features for digital camera applications. These features include a communication processor module (CPM) that supports many communication systems including universal serial bus (USB), a system interface unit that supports realtime application, a 24-bit color video controller, a 24-bit color LCD controller, and a PowerPC processor as the master processor [27]. 


\subsubsection{MPC823's PowerPC Processor Core}

The PowerPC processor core has the following features: 32-bit PowerPC architecture, variable pipeline depth architecture, out-of-order execution termination, branch prediction for prefetch, 32×32-bit general-purpose register file, and precise exception model. These features are implemented in three major units: sequencer unit, register unit, fixed-point unit, and load/store (LD/ST) unit. Figure 3.5 shows the sequencer unit consists of the next address generation unit, the branch unit and the instruction queue unit.

\subsubsection{MPC823's System Interface Unit}

The system interface unit (SIU) provides control of the overall system including external bus arbitration, realtime clock generator, PCMCIA adapter module, and other system functions. The realtime clock generator provides realtime clock for data processing such as transferring data from camera to other devices or implement hardware JPEG standard. On the other hand, the PCMCIA host adapter module provides control logic for PCMCIA interface that is needed for the external memory storage device in digital camera applications. 


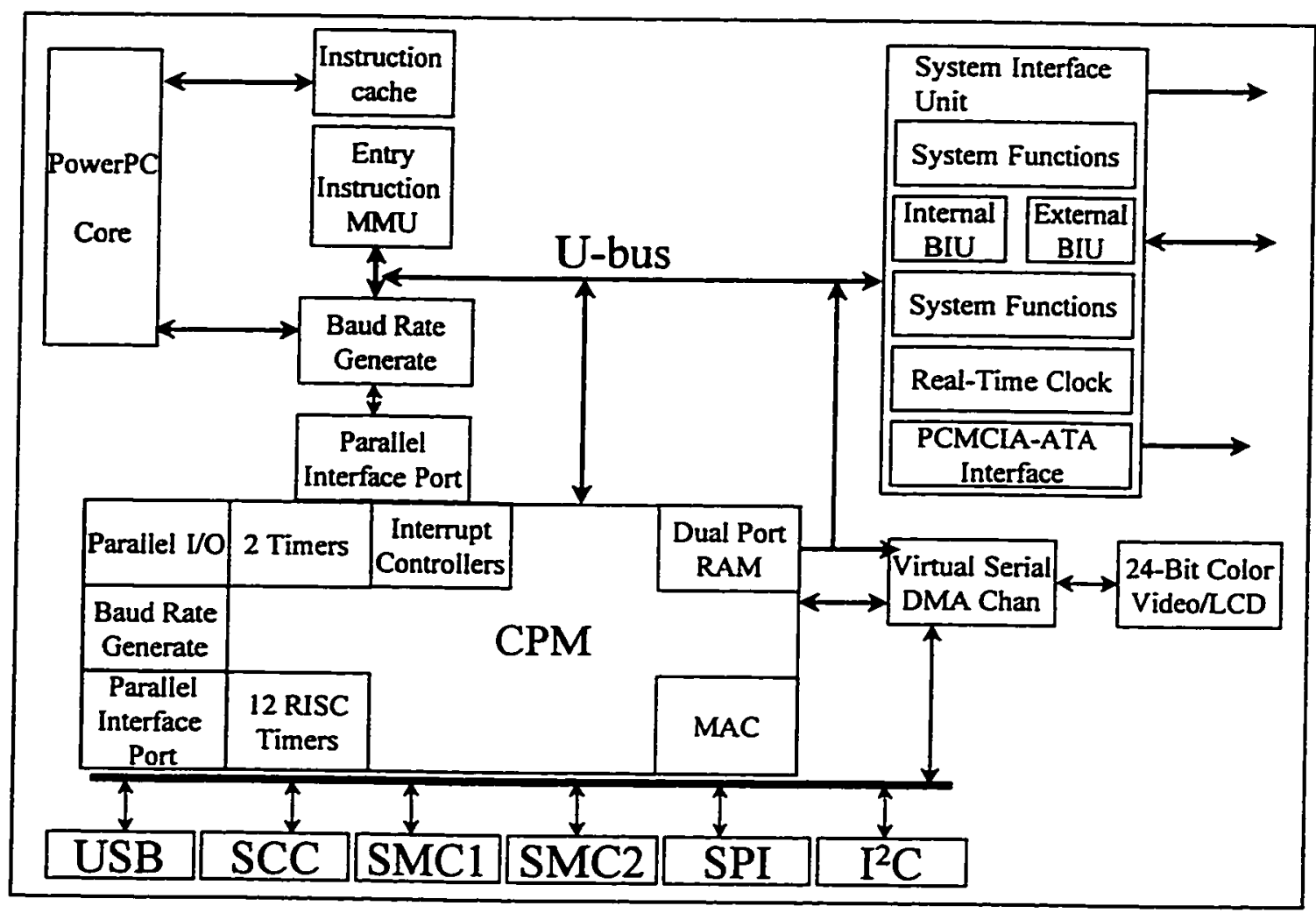

Figure 3.4: Block diagram of the Motorola's MPC 823 processor [27]. 


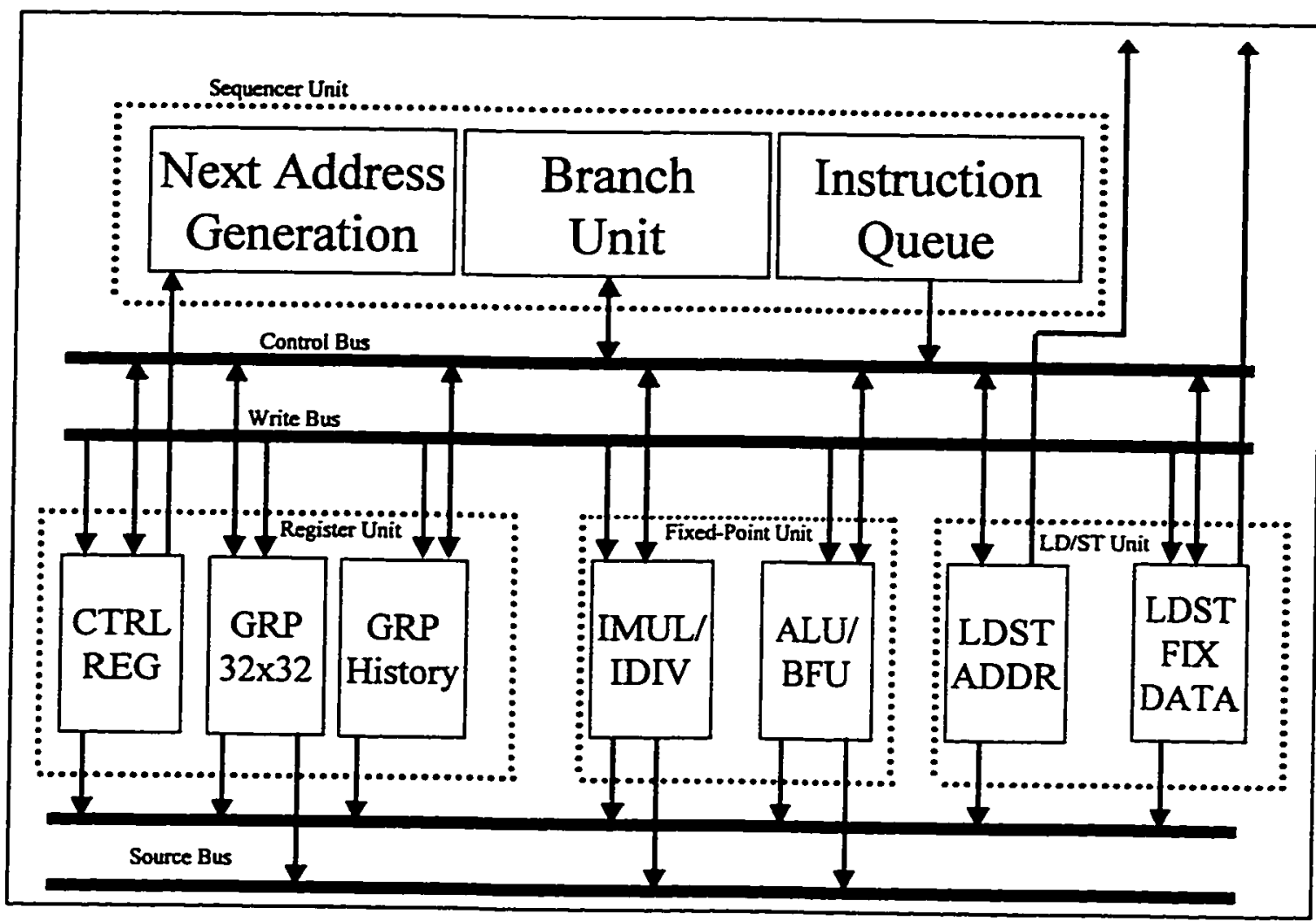

Figure 3.5: Block diagram of the MPC 823's PowerPC Core architectural design [27]. 


\subsubsection{TI's TMS320C80 Architectural Design}

TMS320C80 is one of the latest DSP technologies from Texas Instruments (TI). The TMS320C8x is a family of two versions of RISC core processors: TMS320C80 and TMS320C82. The TMS320C80 has several attractive features that can be use for digital camera applications. These features include the master processor (MP), the four DSP processors, the transfer controller (TC), the video controller (VC), and the crossbar interface that connects all the processors together [28]. Our focus in this section is to discuss the main features of the TMS $32 \mathrm{C} 80$ and its architectural design to provide realtime capability for digital camera applications.

\subsubsection{TMS320C80's Master Processor Architecture}

The MP performs these major tasks: user interface, information processing, and use of IEEE-754 floating point for vector computation. MP accomplishes three major functions efficiently by including a 4-KB instruction cache and a 4-KB data cache. These 4-KB caches are dedicated for the MP use only. Each 4-KB cache is partitioned into four blocks of 256 bytes. The 256-byte block is further partitioned into four sub blocks of 64 bytes each. The 64-byte block holds sixteen 32-bit wide words. This organization allows better handling of cache miss. In addition to the dedicated cache memory, MP also has a 4-KB parameter RAM. MP primarily operates on data that has been brought on-chip by the TC. Also, MP has the capability of accessing off-chip memory by direct external memory access (DEA) which avoids flushing or loading its cache. 


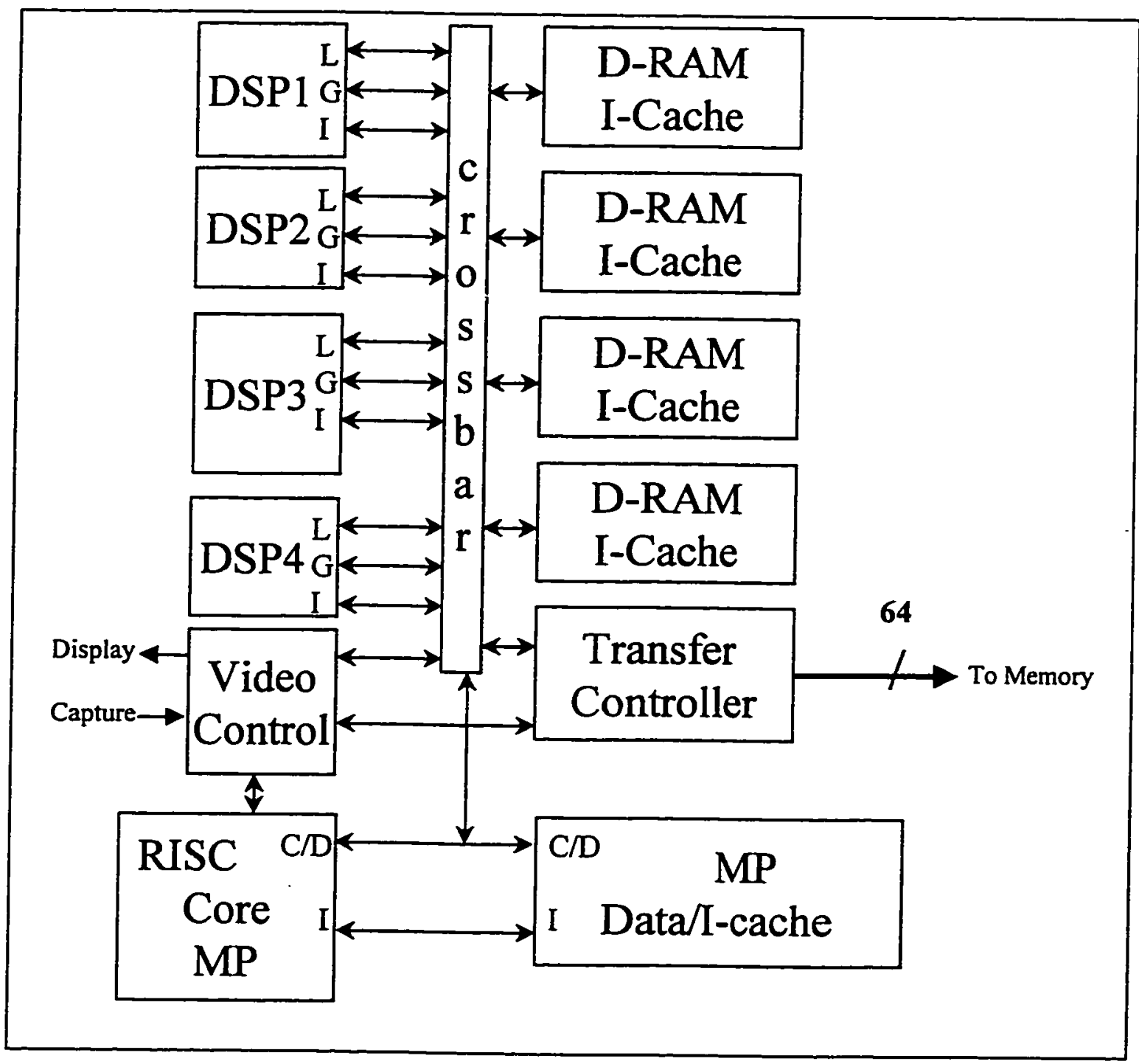

Figure 3.6: Block diagram of the TI's TMS320C80 processor [28]. 
Furthermore, MP uses several pipelines to improve performances. The fetchexecute-access (FEA) pipeline handles parallel instruction in the fetch, execute, and access stages. The fetch stage allows program counter (PC) to fetch the instruction from memory at the address indicated in the PC. The execution stage decodes the instruction and executes the instruction after the instruction is fully decoded. The access stage is use only for load and store instructions for memory access.

Lastly, an important part of the MP architectural design is the floating-point unit. The floating-point unit consists of a floating-point adds/subtracts unit, and multiplication unit which multiply, divide and performs square root computation. To improve floatingpoint precision, both single precision and double precision were implemented. Figure 3.7 shows the IEEE-754 floating-point format implemented in the MP architectural design.

$$
(-1)^{S *(1+\text { significand }) * 2^{\text {(exponent-bias) }}}
$$

\begin{tabular}{|c|c|c|}
\hline 313029282726252423222120,191817161514131211102876543210 \\
$S$ & $\begin{array}{l}\text { Exponent } \\
\text { (11-bits) }\end{array}$ & $\begin{array}{l}\text { Significand } \\
\text { (20-bits) }\end{array}$ \\
\hline
\end{tabular}

Significand (cont'd)

(32-bits)

Figure 3.7: The IEEE floating-point standard format. 


\subsubsection{TMS320C80's DSP Architecture}

The four DSPs are 32-bit processors capable of performing efficient image processing. The key features of the DSPs are the fully programmable of each of the four DSPs, the parallel operation to support up to 64-bit word instructions, and the parallel pipelines. In addition, each DSP has three buses to transfer data among the DSPs. These buses are the local destination/source bus (LDSB), the global source bus (GSB), and the global destination bus (GDS). The LDSB transfers data to/from local ports. The GSB transfers data from the address unit or from the program flow control unit to the ALU's data unit. The GDS transfers data to the destination through the global data port. Furthermore, each DSP has a 2-KB instruction cache that was divided into four sub blocks of 64 bytes each as in the MP's instruction cache.

The DSP's data unit was designed for DCT, FFT, filters, and intensive graphic computation. It has a three-input ALU, a multiplier data path, and register files. In addition, the DSP also have the program flow control unit which implements the fetch, address, execute (FAE) pipeline and all control signals between the TC and DSPs. The fetch stage fetches the instruction from cache and decodes the instruction. The address stage computes the address for the decoded instruction. This address is then sent to the crossbar. At the execute stage, data are fed from the register files, perform the arithmetic operation, and write the result back to the register files. The address unit generates global addresses and local addresses from the global source/destination bus, and the local source/destination bus. 


\subsubsection{TMS320C80 Transfer Controller Architecture}

The transfer controller handles all the data movement among the MP, DSPs, TC, $\mathrm{VC}$ and external devices. The movement of data is in the form of packet transfer. The size of the transfer packet is determined based on the parameter table. Figure 3.9 shows the two parameter tables. For long-form parameter, each packet is a 64-byte and for short-form parameter, each packet is a 16-byte. 


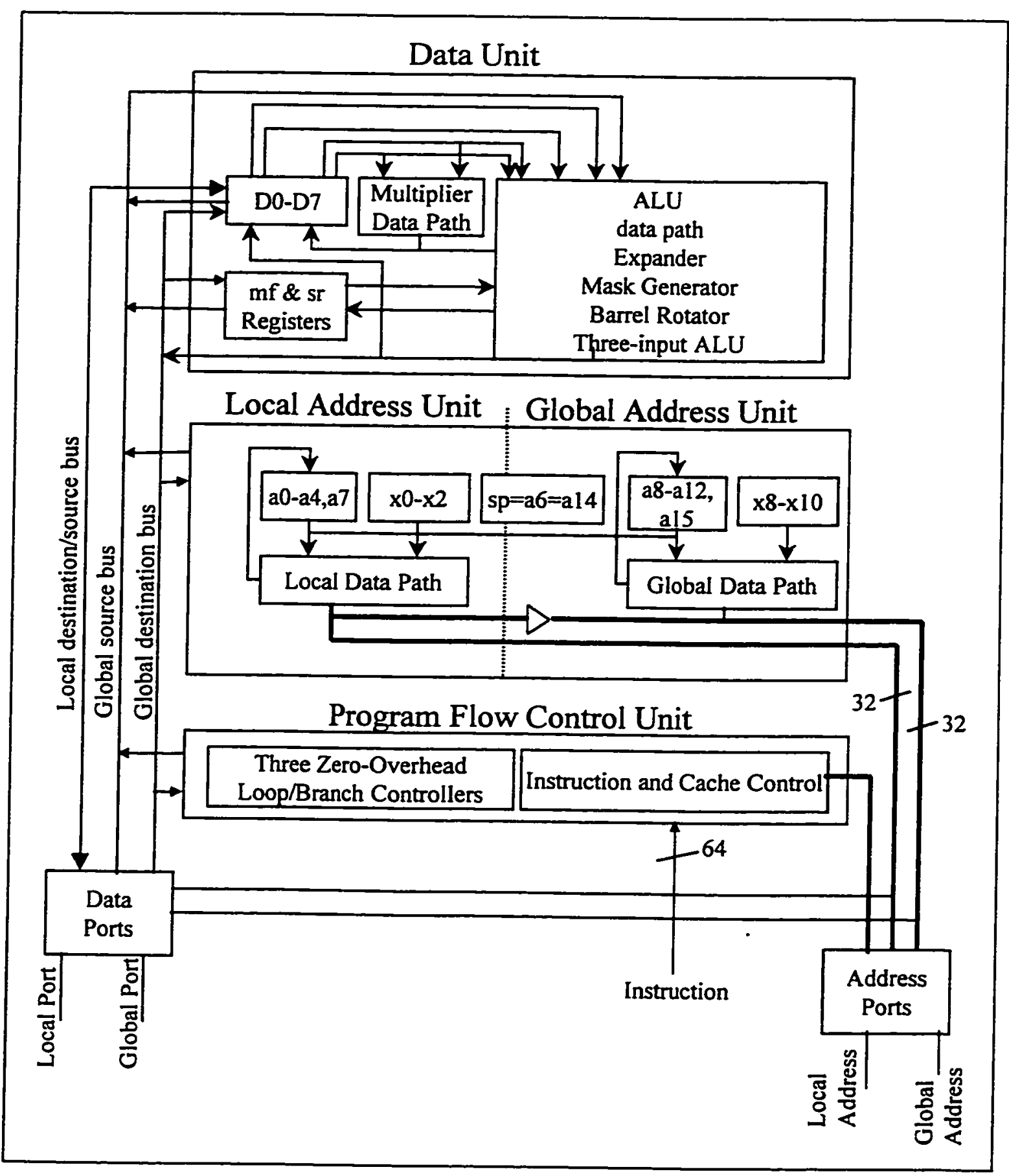

Figure 3.8: Block diagram of the TMS320C80's DSP architectural design [28]. 


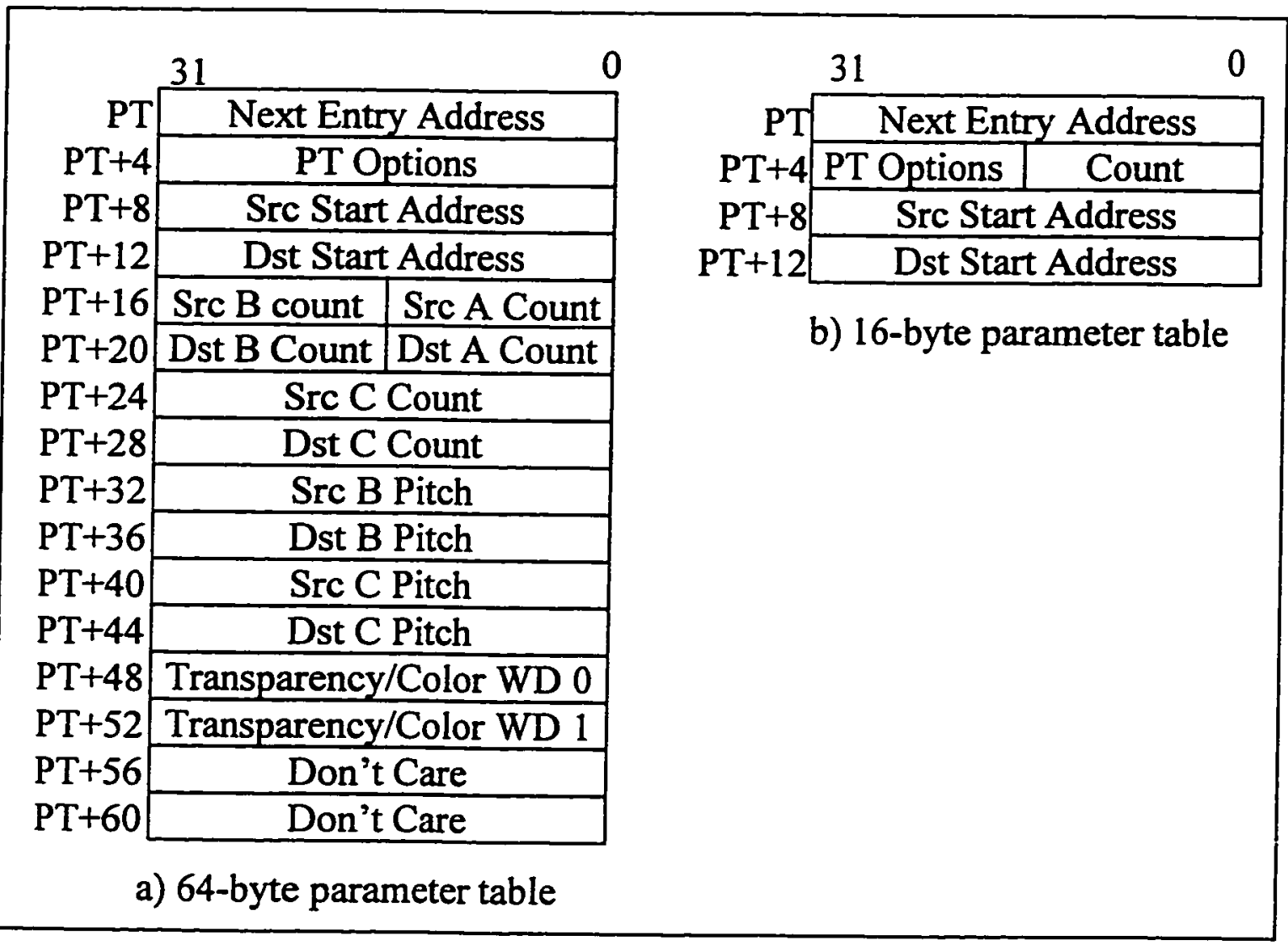

Figure 3.9: The parameter table use by the TMS320C80's TC to handle data movement. (a) Shows the 64-byte use for long packet. (b) Shows the 16-byte use for short packet[28]. 


\subsubsection{TMS320C80's Crossbar Architecture}

In contrast to bus interconnection, which offers the simplest interconnection topology, the TMS320C80 implements the crossbar interconnection topology. Ideally, the crossbar topology provides the highest complexity with the least bus contention. The idea of the crossbar is to connect $\mathrm{N}$ processors to $\mathrm{M}$ memory units with a delay of only one crosspoint. Historically, the aim of this topology is to eliminate bus contention in the communication network [12]. The contention occurs when two processors try to access the same memory location at a given clock cycle. The crosspoint simply connects a processor to a memory unit and should not be considered bottleneck.

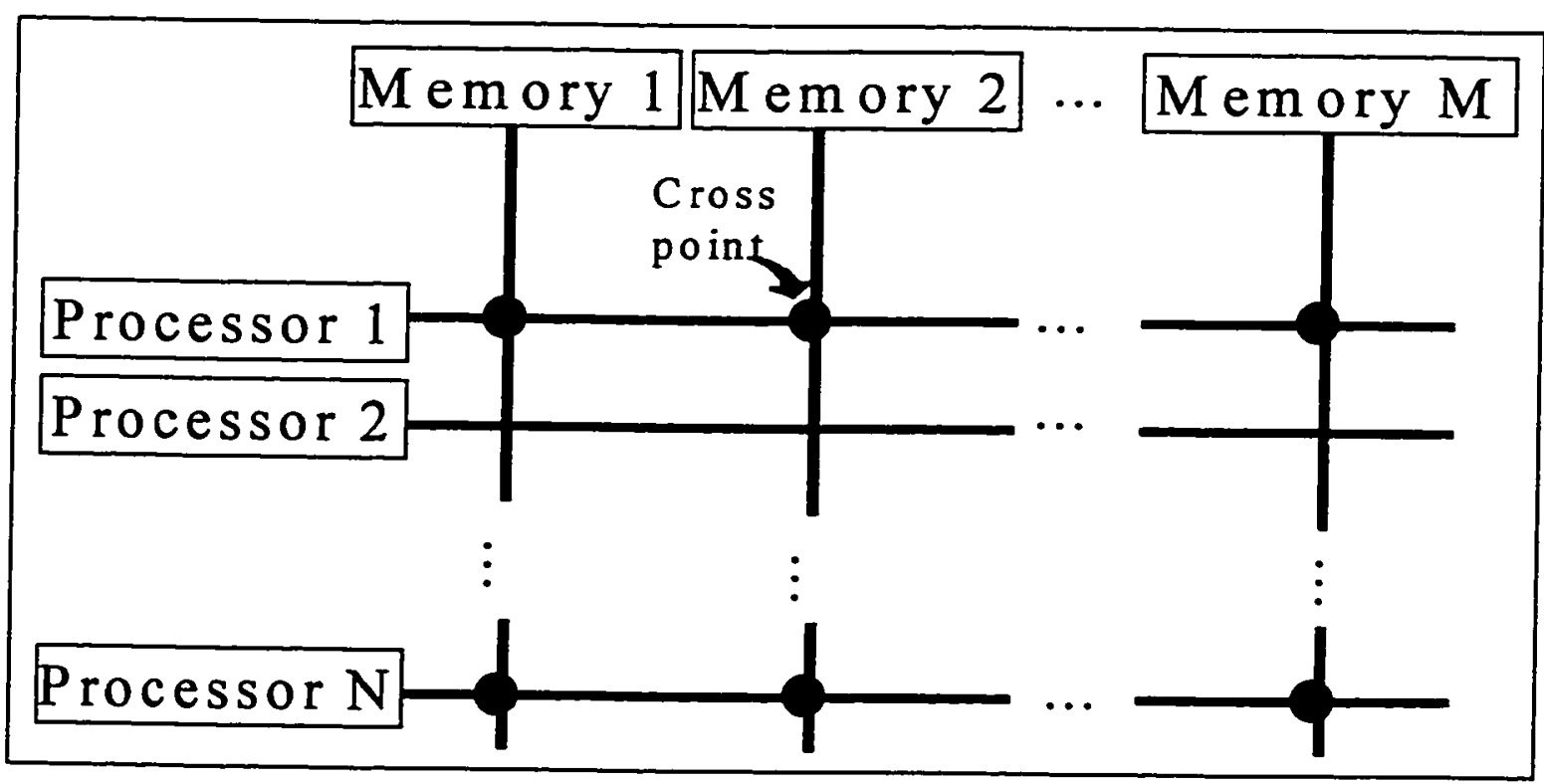

Figure 3.10: An ideal crossbar topology interconnection for $\mathrm{N}$ processors to $\mathrm{M}$ memory units. 
In the TMS320C80 implementation, the crossbar topology is the communication network among the MP, parallel DSPs, TC, and memory units. MP has two ports connected to the crossbar. One is the instruction port and the other is the data port. Both ports provide access to the MP cache. In addition, each DSP has three ports that connect to the crossbar. These are the local data port, the global data ports, and the instruction port. The local data port provides access to the local RAM in DSP. The global data port provides access to the shared memory of the processors. The instruction port provides access to the DSP instruction cache.

In the event that a conflict arises between two processors accessing the same memory location, the conflict is resolve by the priority-scheduling algorithm or by the round-robin scheduling algorithm. The priority-scheduling algorithm simply allows a task with higher priority to access the location first before the lower priority task. Table 3.1 shows the priority table according to a list of tasks.

Figure 3.10 shows the scheme of the round-robin algorithm. The idea of the round-robin-scheduling is to allow access to a cycle equally among all the four DSPs through the use of a token. The token is passed from one DSP port to the other DSP port in a fixed order thus all four DSP processors have equal use of the access. The round robin scheduling can be disabled by using the MP configure register. If the round robin scheduling is turned-off, DSP3's global port always has the token in TMS320C80. 


\begin{tabular}{|l|l|}
\hline Task & Priority \\
\hline $\begin{array}{l}\text { TC pipeline Flush; VC PTs; External } \\
\text { PTs; Cache; DEA, and Urgent PTs }\end{array}$ & 5 (highest) \\
\hline MP & 4 \\
\hline TC High-Priority PTs & 3 \\
\hline DSP & 2 \\
\hline TC Low-Priority PTs & l(lowest) \\
\hline
\end{tabular}

Table 3.1: The TMS320C80 Crossbar's priority arrangement use to schedule task. The priority scheduling algorithm is designed to allow TC pipeline Flush, VC packet transfers (PTs), external PTs, cache, DEA and Urgent PTs to have the highest priority among all tasks. The TC low-priority PTs have the lowest priority.

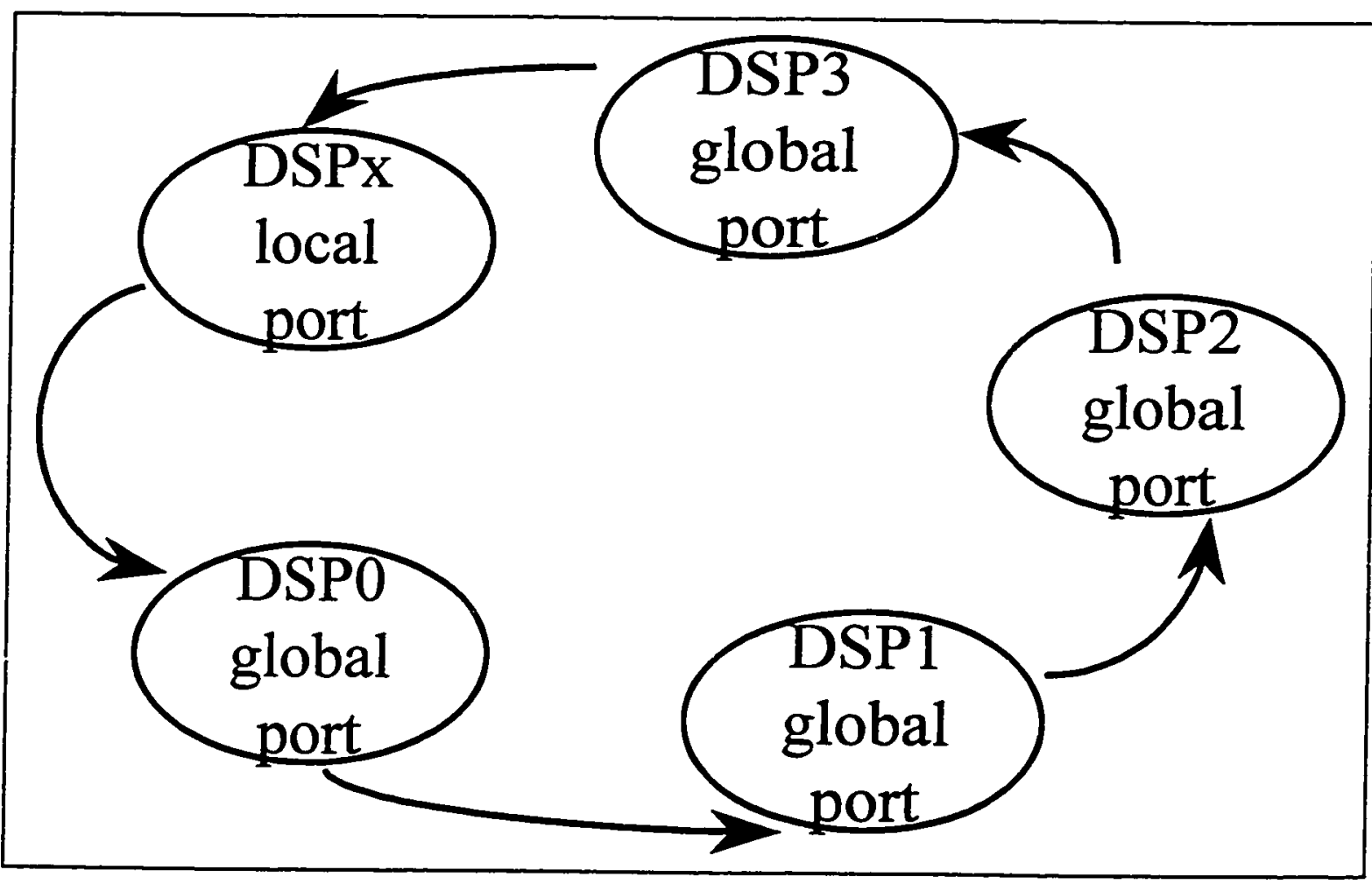

Figure 3.11: The round robin's token passing order this is used to resolve conflict among the DSPs. 


\subsection{The Instruction Set Architecture}

The instruction set of these processors can be categorized into six different classes based on the operator type. Table 3.2 shows the classes with some mnemonic instruction as example. Digital camera applications use mainly the data transfer class and arithmetic operation class. The data transfer class is used to move data from memory to register, register to memory or from register to register. An operator from the arithmetic operation class is performed on the data and the result is transferred to a memory location or to a register by a different instruction in the data transfer class. On the other hand, the system control class provides instruction for the user to program the camera to operate in specific mode of operation such as light exposures, auto focuses, and shutter speeds. Also, the least use instruction class is the branch class. No complex branch instruction design is necessary to determine the destination of the data.

In digital camera implementation, it is not clear which instructions get used the most. However, camcorder implementation in which motion photographic experts group (MPEG) standard is used as the CODEC, the motion vector is the most computation intensive. It requires searching the macroblock and comparing the searched macroblock for possible match (equation 4.1). Thus, each comparison requires five instructions, namely accessing the pixel (two instructions from the data movement class for each operand), a subtraction, an absolute-value calculation, and one addition. The total operations for a macroblock is defined by the following equation [7]: 
(3.1) $\quad \frac{I J F}{M N}(2 P+1)^{2} * M N * 5$

Where $\mathrm{IxJ}$ is the picture resolution

$\mathrm{F}$ is the frame rate

$\mathrm{MxN}$ is the macroblock

$P$ is the search window

For example, a picture resolution of $720 \times 480$ pixels, a frame rate of 30 frames per second, a macroblock of $16 \times 16$ pixels, and a search region of 15 will result in 49.81 giga operations per seconds or 49.81 billion instructions.

\begin{tabular}{|c|c|c|c|c|c|}
\hline $\begin{array}{c}\text { Data } \\
\text { Transfer }\end{array}$ & $\begin{array}{c}\text { Arithmetic } \\
\text { Operation }\end{array}$ & Logic & Shift & Branch & $\begin{array}{c}\text { System } \\
\text { Control }\end{array}$ \\
\hline MOVB & ADD & AND & ROTL & BF & CLRMAC \\
\hline MOVW & SUB & NOT & ROTR & BT & CLRT \\
\hline SWAP & ADDI & XOR & ROTCL & BT/S & LDS \\
\hline
\end{tabular}

Table 3.2 The different classes of instructions. 


\subsection{The I/O Interface Design}

In this section, the I/O interface features of all the architectural designs will be discussed. In evaluating the overall performance, it is essential that VO interface design be part of the embedded architectural design. In particular, I/O designs such as LCD controller, video controller, USB, IEEE 1394 bus, PCMCIA adapter, and other highspeed $\mathrm{I} / \mathrm{O}$ design are the focus in digital camera applications.

\subsubsection{Matsushita Electric Industrial VDSP2's I/O Interface Design}

Unlike other general-purpose processors that have many $\mathrm{I} / \mathrm{O}$ interface design features, the VDSP2 is considered to be a more dedicated image processor with very few I/O design interface features. The VDSP2 has a data I/O processor that has a DRAM port, code bus port, video bus port, and a parallel L/O port. The video port is a FIFO buffer intended to hold data until the display device controller reads it. The data I/O processor can support up to $8 \mathrm{MB}$ of address spaces.

\subsubsection{Hitachi SH-DSP HD643710F's I/O Interface Design}

There are several on-chip I/O interface designs in the SH-DSP processor. These I/O interface designs include the serial communication interface (SCI), serial I/O (SIO), serial debug interface (SDI), bus state controller (BSC), and direct memory access (DMAC). SCI has two channels that operate in asynchronous or synchronous mode to provide full duplex communication to an external device. The SIO has three channels 
that provide full-duplex transfer of data through the ports. The SDI provides debugging interface to the HS-DSP during the prototype phase. The BSC provides address space for external memory through the CS0-CS3 signals. Finally, DMAC provides four channels to allow direct transfer of data to external device without interrupting the CPU.

\subsubsection{LSI Logic DCAM-101's Interface Units}

The LSI Logic's DCAM-101 consisted of several types of I/O interface design. These designs are the CCD interface unit, display interface unit, PCMCIA interface unit, bus interface unit, and serial interface unit. These interface units are interconnected through the internal bus, which then interface to the processor unit through the two-port SRAM.

The CCD interface unit generates all the CCD timing and control signals for the DCAM-101 to connect to a CCD for image capturing. It should be noted that the CCD device is not part of the DCAM-101, only the capability to interface to the CCD device is provided. Also, the CCD interface unit implements basic digital camera function such as digital clamping, shading adjustment, white balancing adjustment and look-up-table (LUT) for quantization. It supports data input of 10-bit CCD or 12-bit at a data rate of 13.5 $\mathrm{MHz}$ or $20 \mathrm{MHz}$ using an internal clock.

Next, the display interface unit includes LCD and NTSC/PAL display. The LCD display unit generates all timing and control signals allowing it to display an 8-bit RGB output format on a LCD screen. On the other hand, the NTSC/PAL display unit 
generates all timing and control signals to allow display in both NTSC and PAL format. It includes 4:4:4, 4:2:2 and 4:1:1 subsamples, and 10-bit programmable video DAC.

The PCMCIA Interface Unit generates all control signals for PCMCIA interface to external memory such as compact flash card. These signals include SYSCLK for Outputs valid, inputs Setup to SYSCLK, inputs Hold from SYSCLK and others.

\subsubsection{Motorola MPC 823's V/O Interface Design}

The MPC 823 has several powerful I/O interface design features. These features include the CPM that has many communication protocols, the video controller, and the LCD controller. All these features are built-in with the MPC 823 processor.

\subsubsection{The MPC823's Communication Processor Module}

The communication processor module (CPM) is a dedicated module that supports external communications. The CPM supports many communication protocols including universal asynchronous receiver transmitter (UART), IEEE 802.3/Ethernet, high level data link control (HDLC), synchronous data link control (SDLC), infrared, USB and others. The USB protocol supports up to four end-points that have plug-and-play capability. In addition to these serial interfaces, the time slot assigner (TSA) adds more support by providing time-division multiplexed (TDM) interface. It includes many formats such as T1 line, pulse code modulation, and others. When used for digital camera applications, these protocols implement direct connection to the Internet or 
wireless data transfer to external devices such as printers.

\subsubsection{MPC823's LCD Controller}

The LCD controller provides up to 256 color active matrix displays. The LCD controller consisted of a pixel generation block, a controller block, FIFO block, register block, and timing generator. The primary function of the pixel generator block is to transfer data from the FIFO to the LCD interface block. This includes the gray scale or color pixel to be transferred to the frame buffer. The controller block consists of the DMA controller, the frame controller, the vertical controller, and the horizontal controller. The DMA controller controls the filling of the FIFO block with data from the DMA interface. The frame controller controls the frame rate and the counters of the DMA and the vertical control block. The vertical controller controls the line pixels in the vertical column and activates the horizontal control to increment the line scan. Similarly, the horizontal controller controls the horizontal line scans and signals the vertical block when done with its line scan.

\subsubsection{MPC823's Video Controller}

The video controller (VC) can be programmed to display on LCD screen or NTSC/PAL video signals. The VC architecture consists of a FIFO unit, a DMA control, a configuration registers, a video control RAM (VCR), and a MUX. The VCR contains two RAMs of 64 entries that are 32-bit wide. One of the two RAMs is an active RAM 
that stores the active video parameters while the other serves as a shadow RAM. Each entry in the RAM stores the following parameters: horizontal synchronous on rising edge, horizontal synchronous on falling edge, vertical synchronous on falling edge, vertical synchronous on rising edge, blanking on falling edge, blanking on rising edge, video data select (VDS), interrupt, loop cycle/clock count, and other fields. All these fields are programmable.

On the other hand, the configuration registers hold the mode and configuration bit for the video controller. These configurations include data timing, data polarity, default pixels format, and other relevant parameters in the video controller.

Lastly, the MUX allows data to be displayed from the FIFO, configuration registers, or VCR. Data from the FIFO are considered active pixels while data from the configuration registers are considered to be background pixels. 


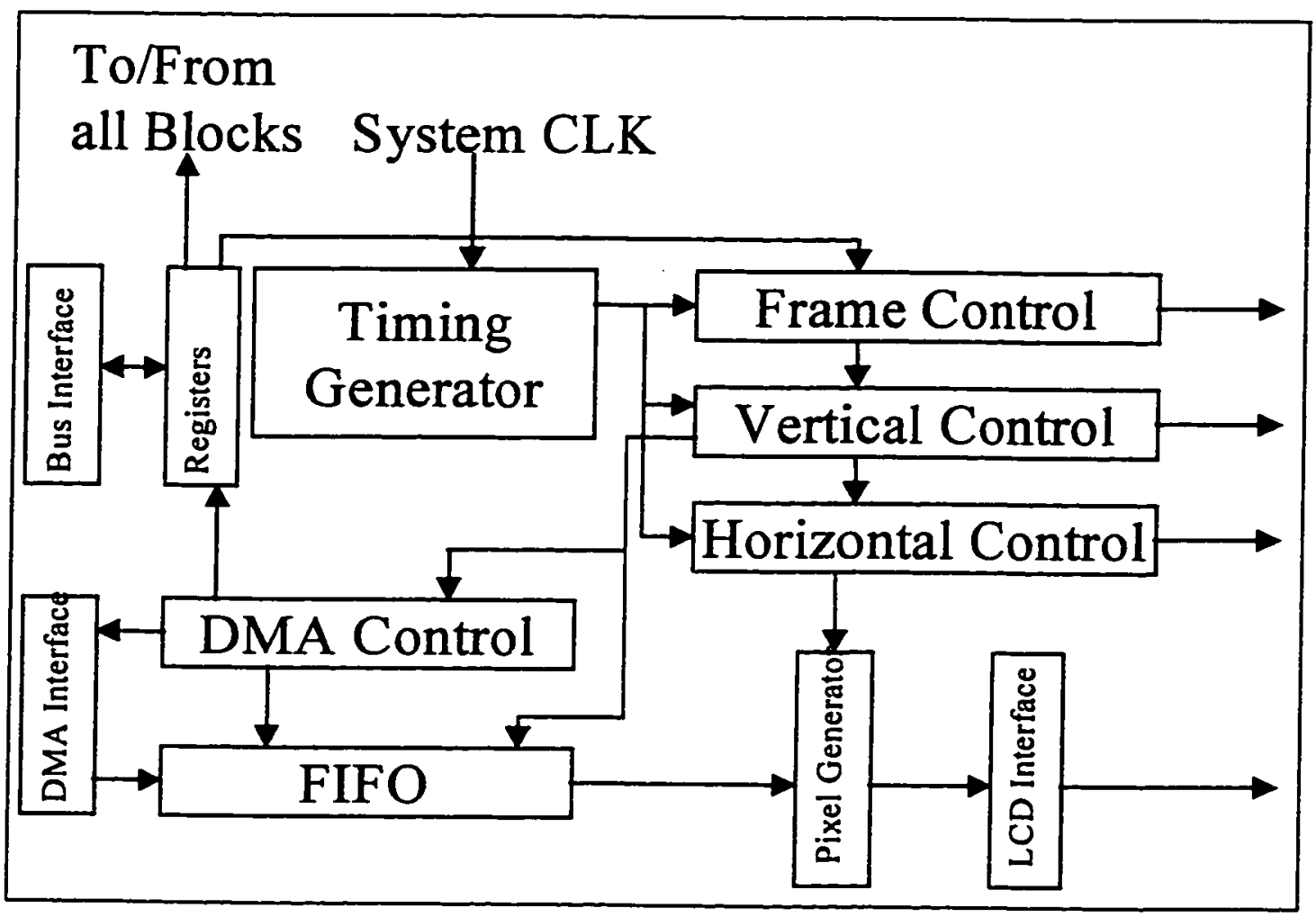

Figure 3.12: Block diagram of the MPC823's LCD controller architectural design. 


\subsubsection{TMS320C80's Video Controller}

The primary function of the video controller (VC) architecture is to provide video interface to external devices such as TVs, LCD displays, printers, copiers, or scanners. The VC consists of the serial register transfer (SRT) controller, the frame timer, and the VC register-interface. The SRT controller generates SRT cycle requests to the TC to transfer data into and out of VRAM frame memories. The frame timer generates timing control for synchronization of frame. The VC register-interface provides data transfer between the MP register and the VC. 


\subsection{The Benchmarks of the Performance}

The architecture of the VDSP2 is a 0.5 -micron CMOS technology. It operates at $100 \mathrm{MHz}$ with a maximum bit rate of $497 \mathrm{Mbit}$ per second. The CPU operates at 200 MIPS while the four DSPs each operates at 200 MIPS. According to reference [17], MPEG2 can be implemented using two VDSP2 processors. Table 3.4 shows an estimated execution time for encoding MPEG-2 using the VDSP2 processor. The processing time for each process is under the 25.5 microseconds that was provided for realtime video encoding by the ITU-601. The motion compensation (MC) is implemented using the Matsushita Electric's VDSP1 processor [16]. All other processes in table 3.4 are implemented using the VDSP2 processor.

The Hitachi's SH-DSP architecture is a 0.35-micron technology with the DSP unit performance of 120 MOPS at $60 \mathrm{MHz}$ and the RISC CPU performance of 60 MIPS. The SH-DSP compresses an image of $640 \times 480 \mathrm{VGA}$ in 0.36 second through software compression.

The LSI Logic's DCAM-101 is the only single chip processor designed specifically for digital camera prototype. It is capable of compressing 3.3 million pixels per second or eleven images of $640 \times 480$ VGA per second through its JPEG hardware unit. The DCAM-101's CPU operated at 84 MIPS.

The Motorola's MPC823 has a performance of 66 MIPS at 50MHz for the CPU. The DSP unit has a performance of 50 MIPS at $50 \mathrm{MHz}$.

The TI's TMS320C80 is a 0.5 -micron CMOS technology. It is capable of 
executing $50 \mathrm{MIPS}$ at $50 \mathrm{MHz}$ for the 32-bit RISC. It has four DSP units with 50 MIPS at $50 \mathrm{MHz}$ per DSP. The floating-point executes at 100 mega floating-point operations per second (MFLOPS).

\begin{tabular}{|l|c|c|c|c|}
\hline Processor & $\begin{array}{c}\text { Technology } \\
(\mu \mathrm{m})\end{array}$ & $\begin{array}{c}\text { Max CLK Rate } \\
(\mathrm{MHz})\end{array}$ & \multicolumn{2}{|c|}{ Performance } \\
\cline { 4 - 5 } & & & CPU & DSP \\
\hline $\begin{array}{l}\text { Matsushita Electric's } \\
\text { VDSP2 }\end{array}$ & 0.5 & 100 & 200 MIPS & 200 MIPS \\
\hline Hitachi's SH-DSP & 0.35 & 60 & 60 MIPS & 120 MOPS \\
\hline $\begin{array}{l}\text { LSI Logic's } \\
\text { DCAM-101 }\end{array}$ & 0.35 & 85 & 85 MIPS & - \\
\hline $\begin{array}{l}\text { Motorola's } \\
\text { MPC 823 }\end{array}$ & 0.35 & 50 & 66 MIPS & 50 MIPS \\
\hline $\begin{array}{l}\text { TI's } \\
\text { TMS320C80 }\end{array}$ & 0.5 & 50 & 50 MIPS & 50 MIPS \\
\hline
\end{tabular}

Table 3.3: A comparison of the performance for the evaluated processors. Also shown is the technology used to fabricate the processors and the maximum clock rate for each individual processors. 


\begin{tabular}{|l|c|}
\hline Process & Time $(\mu \mathrm{sec})$ \\
\hline MC & 19.48 \\
\hline Q/Q & 19.40 \\
\hline VLC & 16.00 \\
\hline VLD & 17.17 \\
\hline DCT/KDCT & 7.96 \\
\hline
\end{tabular}

Table 3.4: Execution time for MPEG2 encoder using Matsushita Electric's VDSP2 processor [25-26].

\begin{tabular}{|l|c|}
\hline Processor & Instructions/pixel \\
\hline Matsushita Electric's VDSP2 & 86.80 \\
\hline Hitachi's SH-DSP & 6.51 \\
\hline LSI Logic's DCAM-101 & 9.22 \\
\hline Motorola's MPC 823 & 7.16 \\
\hline TI's TMS320C80 & 21.70 \\
\hline
\end{tabular}

Table 3.5: An illustration of the processors' MIPS bandwidth to do image processing. The illustration is based on $640 \times 480$ image at 30 frames per second. Based on this, the VDSP2 has the highest MIPS bandwidth for image processing. 


\section{CHAPTER IV \\ THE SUGGESTED ARCHITECTURAL FOR HIGH SPEED HIGH PERFORMANCE DIGITAL CAMERA}

There are many general-purpose processors beside the ones evaluated in chapter 3 for digital camera applications. Most of them are considered overkill in image processing capabilities while there is not enough support in LO capabilities or vice versa. For example, among them is the Motorola MPC823 that is equipped with many useful I/O interface capabilities, including the USB bus, but it does not have a dedicated processor for image CODEC processing. In contrast, the Matsushita Electric Industrial's VDSP2 processor is equipped with four individual VPs for image CODEC processing but does not have high speed I/O interface capabilities. The suggested architecture makes adjustment based on the need for image CODEC, CPU processing, and high-speed I/O interface design for digital camera applications.

\subsection{The Architectural Overview}

The suggested architecture bases many similarities with the LSI Logic's DCAM101 design. However, it extends new features to meet future technology development. These new features include: (1) a CODEC unit that is capable of performing JPEG or MPEG compression standard, (2) a CPM unit that includes USB, wireless infrared communication and IEEE 1394 bus, and (3) a CPU that consists of a 16-bit or 32-bit RISC controller with specific instruction sets for digital camera applications. 


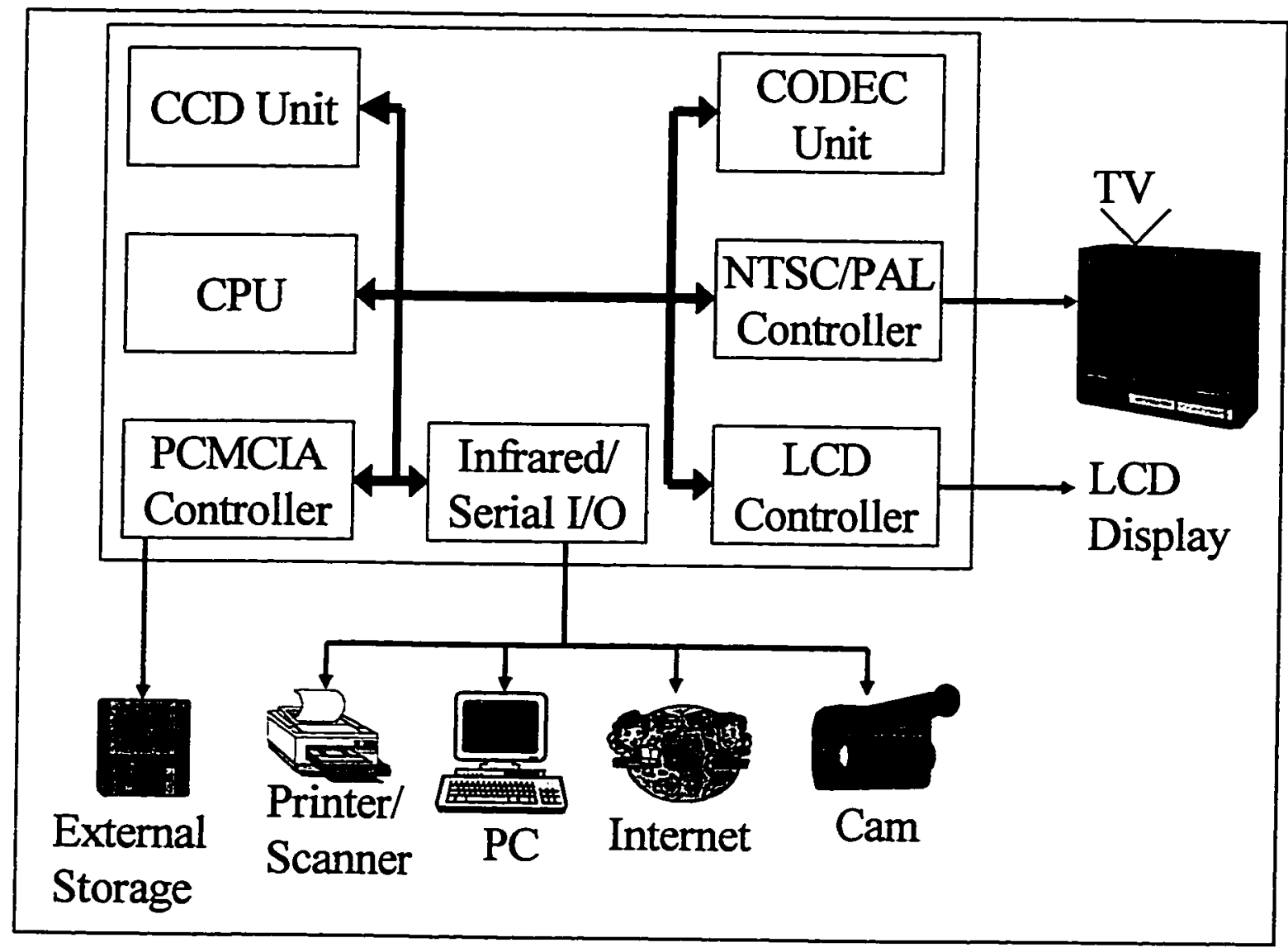

Figure 4.1: The suggested architecture's functional block diagram. 


\subsubsection{The Suggested CODEC Unit}

Image compression is one of the key technologies that make digital camera affordable in the consumer market today. Currently, digital cameras use JPEG standard to compress the image. JPEG standard is limited to still digital camera application. Recently, the technology trend indicated that the convergence of television (TV) broadcasting, PC computing, and Internet applications all demand digital cameras to be both still and motion digital cameras to meet the end users' desire in multimedia applications. The suggested architecture includes the CODEC to perform either JPEG or moving pictures expert group (MPEG) image compression and decompression to meet these desires.

MPEG-1 standard was adopted as international standard in 1991 for the coded representation of moving pictures and associated audio information on digital storage media for up to $1.5 \mathrm{Mbit} / \mathrm{s}$. In 1990, MPEG-2 was initiated to allow greater input flexibility and higher data rates up to $10 \mathrm{Mbit} / \mathrm{s}$. MPEG-3 was suggested to operate at 40 Mbit/s but with small modification to MPEG-2, this data rate can be achieved. Thus, MPEG-3 was not adopted as an International Standard. MPEG-4 is currently being adopted as international standard. MPEG-4 is based on integration of natural and synthetic audio and video material. The coded representation of image is encoded at the lower layers while the application developers do not need to worry about those layers. MPEG-4 is scheduled to be adopted as international standard in November 1998. Besides MPEG-4, plans for MPEG-7 are under way. Currently, the technology trend for 
multimedia applications indicates a high demand for MPEG-2.

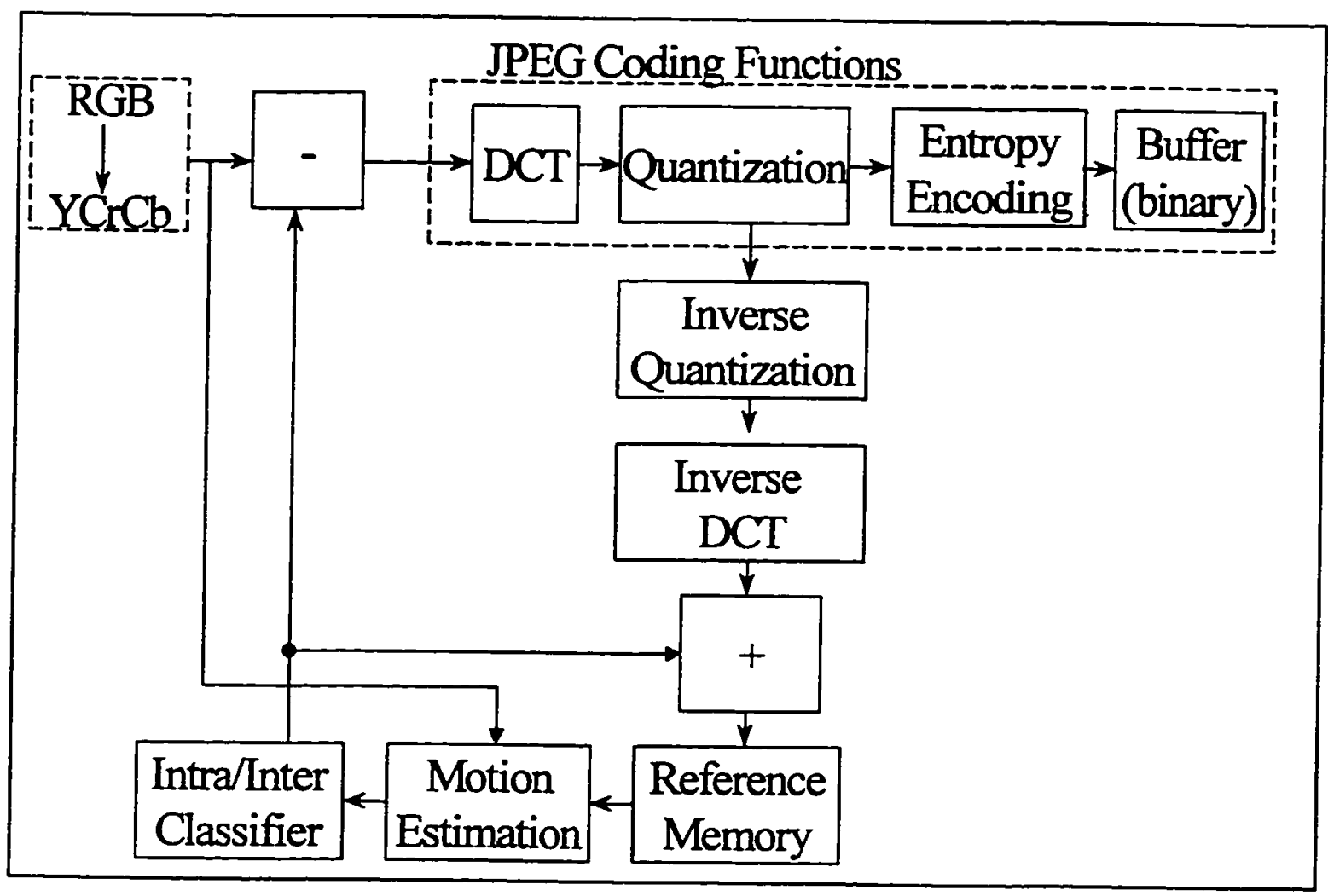

Figure 4.2: Block diagram of video compression in the JPEG and MPEG International Standards. The dotted blocks are the JPEG standard. Both the dotted blocks and solid blocks are the MPEG standard. 
MPEG-2 supports all MPEG-1 features plus the following added features: (1) supports interlace and non-interlace, (2) supports 4:2:0, 4:2:2, and 4:4:4 subsamples, (3) motion compensation based on interlaced or non-interlace frames, (4) options on quantization or alternate ziz-zag ordering, and (5) new syntax for scalable bit streams. Table 4.1 shows the color components of each subsample. The first number in each subsample is associated with the luminance, $\mathrm{Y}$ component, and the last two numbers are associated with the chrominance, $\mathrm{Cr}$ and $\mathrm{Cb}$ components respectively. Figure 4.3 shows the macroblock layout for each subsample. These macroblocks are $8 \times 8$ pixels. With the negotiation option for scalable extension in MPEG-2, these macroblocks can be further scaled to $1 \times 1,2 \times 2$, or $4 \times 4$ pixels. The DCT performs image reduction on these macroblocks.

MPEG-2 encodes on Intra-frame (I-frame), predictive frame ( $P$-frame) and bidirectional frame (B-frame) to obtain high compression ratio. The I-frame does not reference any other frame in the coded bit stream. The P-frame references the I-frame. The B-frames references both the I-frame and the P-frame. How these frames are coded will be discussed after we have discussed the motion estimation block.

A major block of the MPEG standard is the motion estimation. Motion estimation estimates the error between a current frame and a reference frame. This error is called motion vector. The motion vector is needed to exploit the temporal correlation between frames. Figure 4.4 shows the process of finding the motion vector. This process is referred to as the full search method. It uses a macroblock in the current frame to search 
for minimum error in the reference frame. The full search method can be characterized by the following equation which, is known to be the mean absolute error (MAE).

$$
\operatorname{MEAE}(i, j)=\frac{1}{M N} \sum_{k=0}^{M-1} \sum_{l=0}^{N-1}|C(x+k, y+l)-R(x+i+k, y+j+l)|
$$

Where $i$ and $j$ are defined in $-p \leq i \leq p$ and $-p \leq j \leq p$.

Other search techniques are discussed in [7]. The motion estimation has been the most costly in terms of CPU time, which was estimated to take up to sixty percent of the total encoding time.

\begin{tabular}{|c|c|c|c|}
\hline \multirow{2}{*}{ Subsamples } & \multicolumn{3}{|c|}{$\begin{array}{c}\text { Color component } \\
\text { (pixels per line) }\end{array}$} \\
\cline { 2 - 4 } & $\mathrm{Y}$ & $\mathrm{Cr}$ & $\mathrm{Cb}$ \\
\hline $4: 4: 4$ & $720 \times 480$ & $720 \times 480$ & $720 \times 480$ \\
\hline $4: 2: 2$ & $720 \times 480$ & $360 \times 480$ & $360 \times 480$ \\
\hline $4: 2: 0$ & $720 \times 480$ & $360 \times 240$ & $360 \times 240$ \\
\hline
\end{tabular}

Table 4.1: The sizes of each subsample in MPEG-2. The $Y$ component is the same for all three subsamples. The $\mathrm{Cr}$ and $\mathrm{Cb}$ components are reduced according to the ratios. 


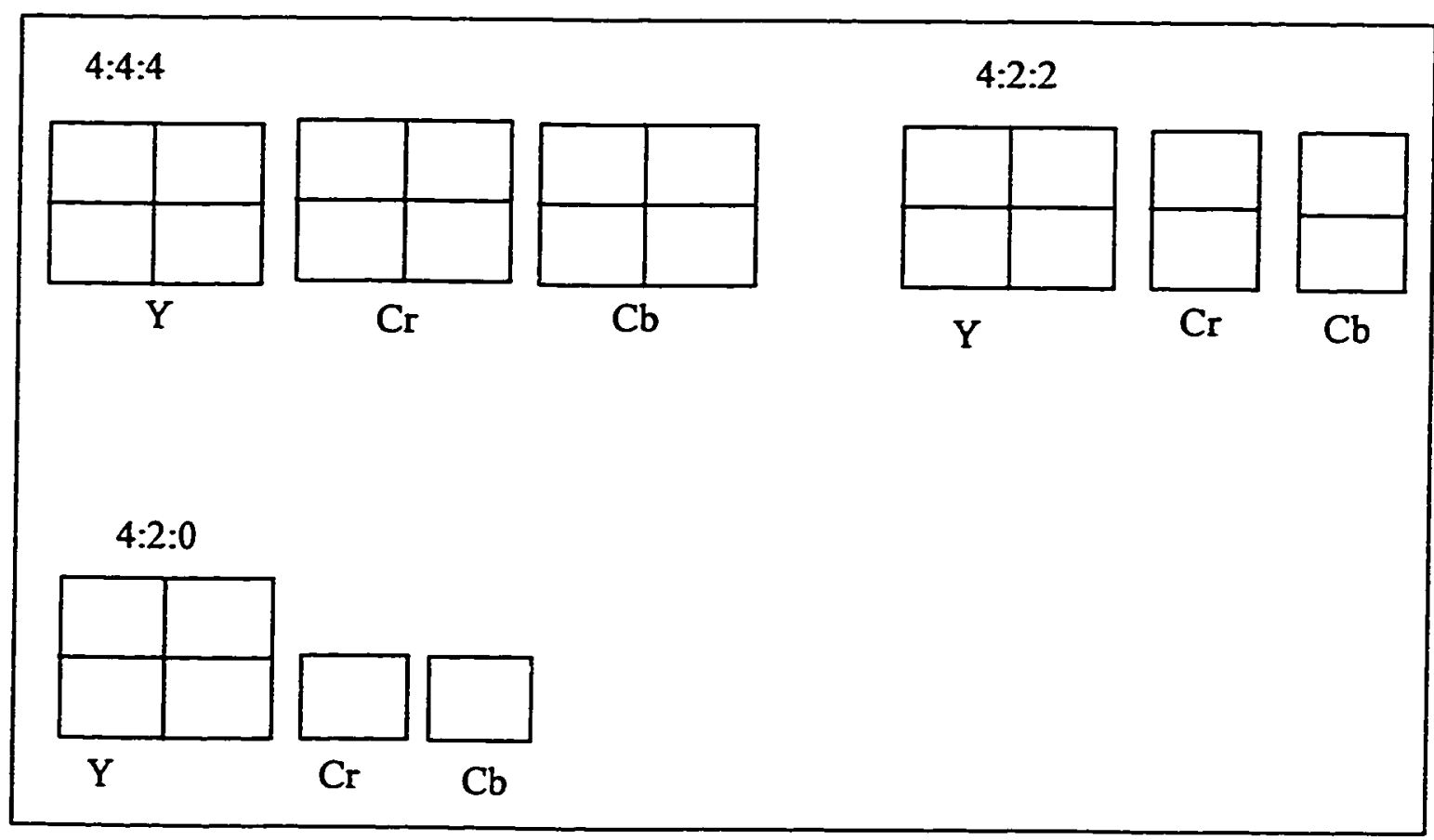

Figure 4.3: Microblock of the luminance and chrominance in the MPEG's subsample. Each square corresponds to $8 \times 8$ square pixels. The diagram shows the relationship of the squares to its subsample ratios for the luminance and chrominance components. 


\section{Current Picture}

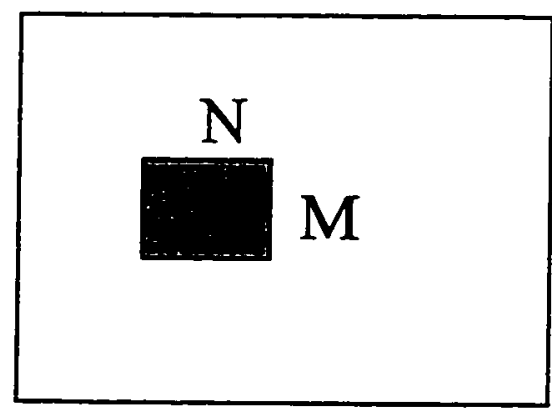

(A)

\section{Reference Picture}

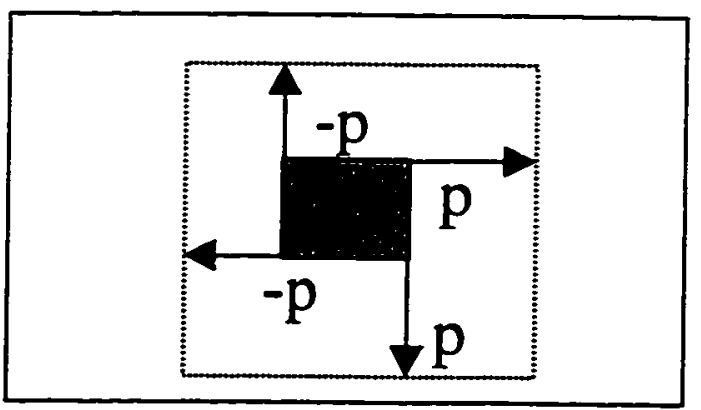

(B)

\section{Reference Picture}

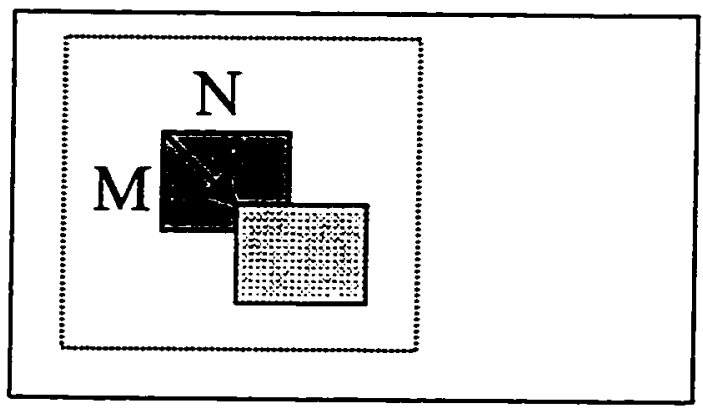

(C)

Figure 4.4: The process to find the motion vector using a full search algorithm. (a) Shows the macroblock in the current frame. (b) Shows the search region that is defined by [-p,p] in the reference frame. (c) Shows the motion vector as a displacement between the current macroblock and the best possible macroblock in the reference frame. 
The encoding process of MPEG-2 follows the blocks in figure 4.2. The Intraframe (I-frame) does not reference any frame so it goes straight to the DCT block to be transformed. The output of the DCT block gets quantized and then goes to the VLC encoder to be encoded into bits as described in chapter 2. Also, a copy of the output from the quantizer goes to the inverse quantization and inverse DCT to be stored in memory for references by Predictive-frame (P-frame) and Bidirectional-frame (B-frame).

On the other hand, the P-frame is coded using information from the I-frame. Thus, each macroblock in the P-frame is subtracted from a motion vector computed using I-frame. The predictive error is the result of the subtraction and is fed into the DCT to be transformed. The output of the DCT block goes through the Q, IQ, IDCT, and VLC in the same manner as the I-frame explained above. Figure 4.5 shows the motion compensation for the P-frame.

Similarly, the B-frame uses information from the I-frame and the P-frame to encode each macroblock. For each macroblock in the B-frame, two motion vectors are needed to form the prediction error. One motion vector is from the I-frame and the other from the P-frame. The prediction error can be formed from either of these two motion vectors or the average of the two motion vectors. The predictive error goes through the same blocks from here onward. Figure 4.6 shows the motion compensation for the Bframe. 


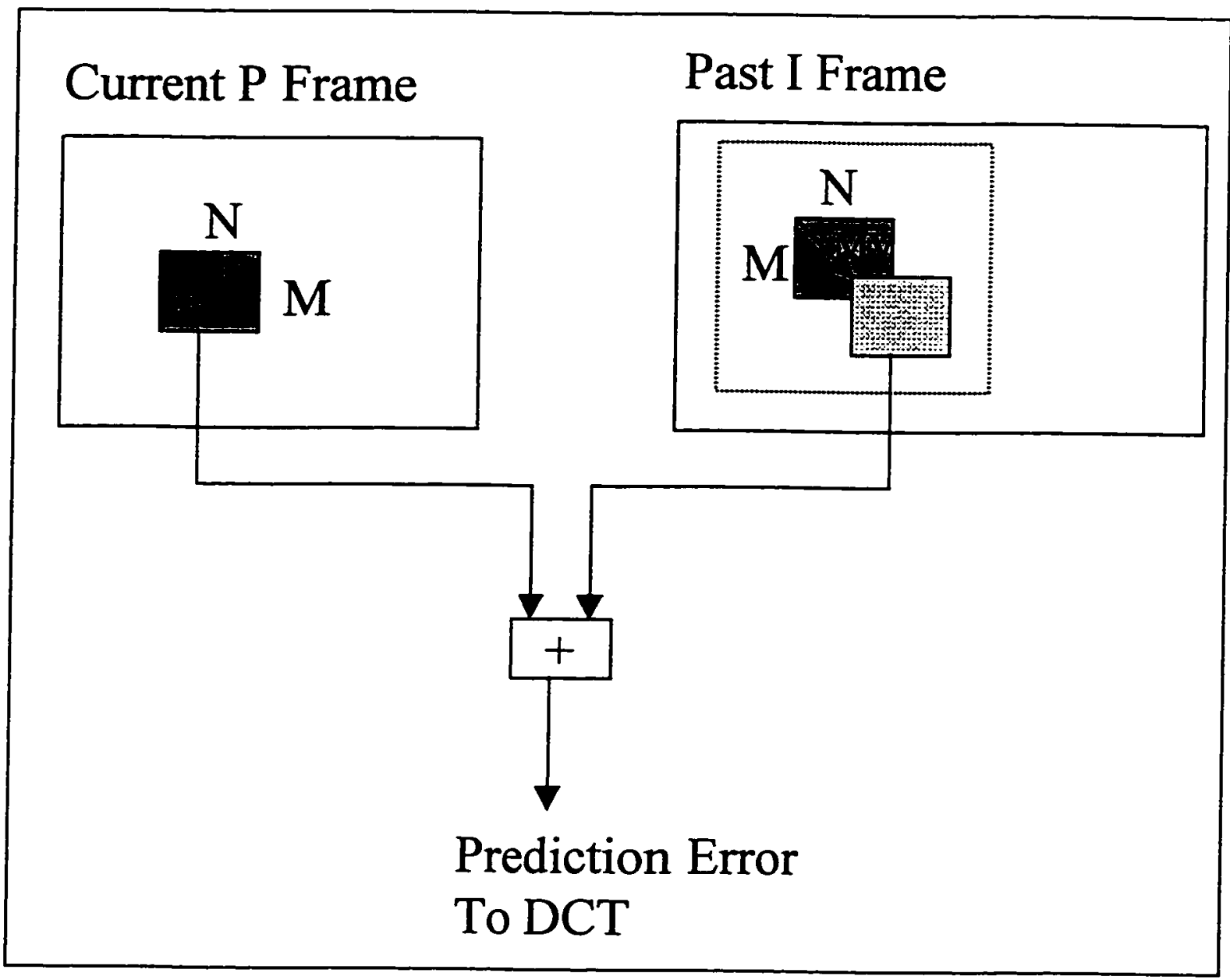

Figure 4.5: Motion compensation for the $\mathrm{P}$-frame. The prediction error can be subtracted from I-frame. 


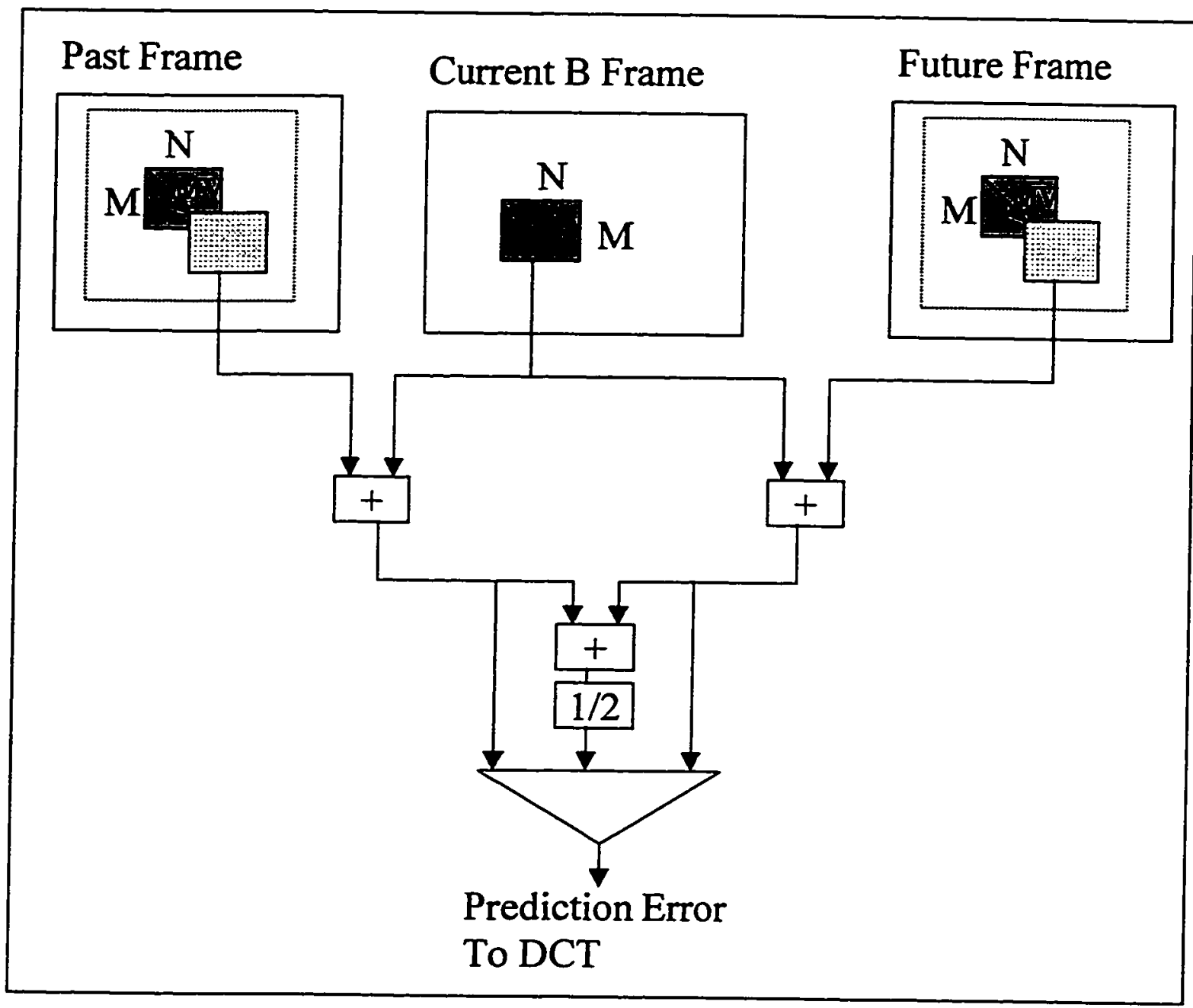

Figure 4.6: Motion compensation for the B-frame. The prediction error can be subtracted from either the P-frame or the I-frame or average of both the P-frame and I-frame. 


\subsubsection{The Suggested CPM Unit}

The communication processor module (CPM) for digital camera applications includes state-of-the-art multimedia communications. These multimedia communications are (1) universal serial bus (USB), (2) IEEE 1394, (3) wireless infrared communication, (4) high-speed UART, and (5) LCD and NTSC/PAL controller.

The USB is a serial bus that bridges the plug-and-play gap between PC and peripheral devices. It was developed by Compaq, Digital Equipment Corporation, IBM PC Company, Intel, Microsoft, NEC, and Northern Telecom. The main focus of the USB is to support all PC peripherals including monitors, audio I/O devices, telephones, modems, keyboards, mice and others which provides a plug-and-play capability without worrying about device addressing and memory mapping. It connects up to 127 devices, supports transfer rate up to $12 \mathrm{Mbit} / \mathrm{s}$, provides full support for the realtime data for voice, audio, and compressed video. Also, it supports mixed-mode of isochronous and asynchronous messaging. The USB bus topology is a pyramid or a tree shape as illustrated in figure 4.5. The top level of the pyramid is the root controller. The second level is a hub with several children as the devices and a child to the next hub level. The third hub level repeats this hub and children topology up to 127 devices.

The connection between a device and the host is established in three layers. Figure 4.7 illustrates these three layer connections. The interface layer is the actual connection in which the physical median connects the device interface to the host controller. The device layer is the next layer above the interface layer. The device layer 
talks to the USB system SW logically. The top layer is the function layer in which the device function talks to the client SW in the host. This layer connection is similar to many Internal Standards Organization (ISO) Open Systems Interconnection (OSI) reference models, including the TCP/IP protocol.

Another serial communication bus is the IEEE 1394, also known as the FireWire. Unlike the USB, the IEEE 1394 is targeting at high-speed peripheral such as camcorders, digital cameras, and digital videodisc players. It has a transfer rate of $400 \mathrm{Mbit} / \mathrm{s}$, and capable of connecting up to 63 devices. It supports both asynchronous and isochronous messaging mode and supports realtime communication with average of a new cycle every 125 microsecond. The IEEE 1394 is a point-to-point network with a maximum cable length of 4.5 meters between each hop. It has a maximum of 16 hops that a signal may be passed through.

Yet, other I/O interface communications in the CPM are the wireless infrared communication and the high-speed UART. These communications allow the CPM to be used in a wide variety of applications such as direct Internet connections, PC connections, camera-to-camera connections, and camera-to-printer connections. Wireless infrared communication and high-speed UART are considered to be standard I/O interface design in digital camera applications.

Last but not least, the LCD controller and the NTSC/PAL controller are also part of the CPM unit. Most high-end digital cameras include a 24-bit to 36-bit pixel depth for LCD controller and NTSC/PAL controller. The suggested architecture takes an average 
of this and a 30-bit pixel depth for the LCD controller and NTSC/PAL controller are sufficient for future camera architectural design.

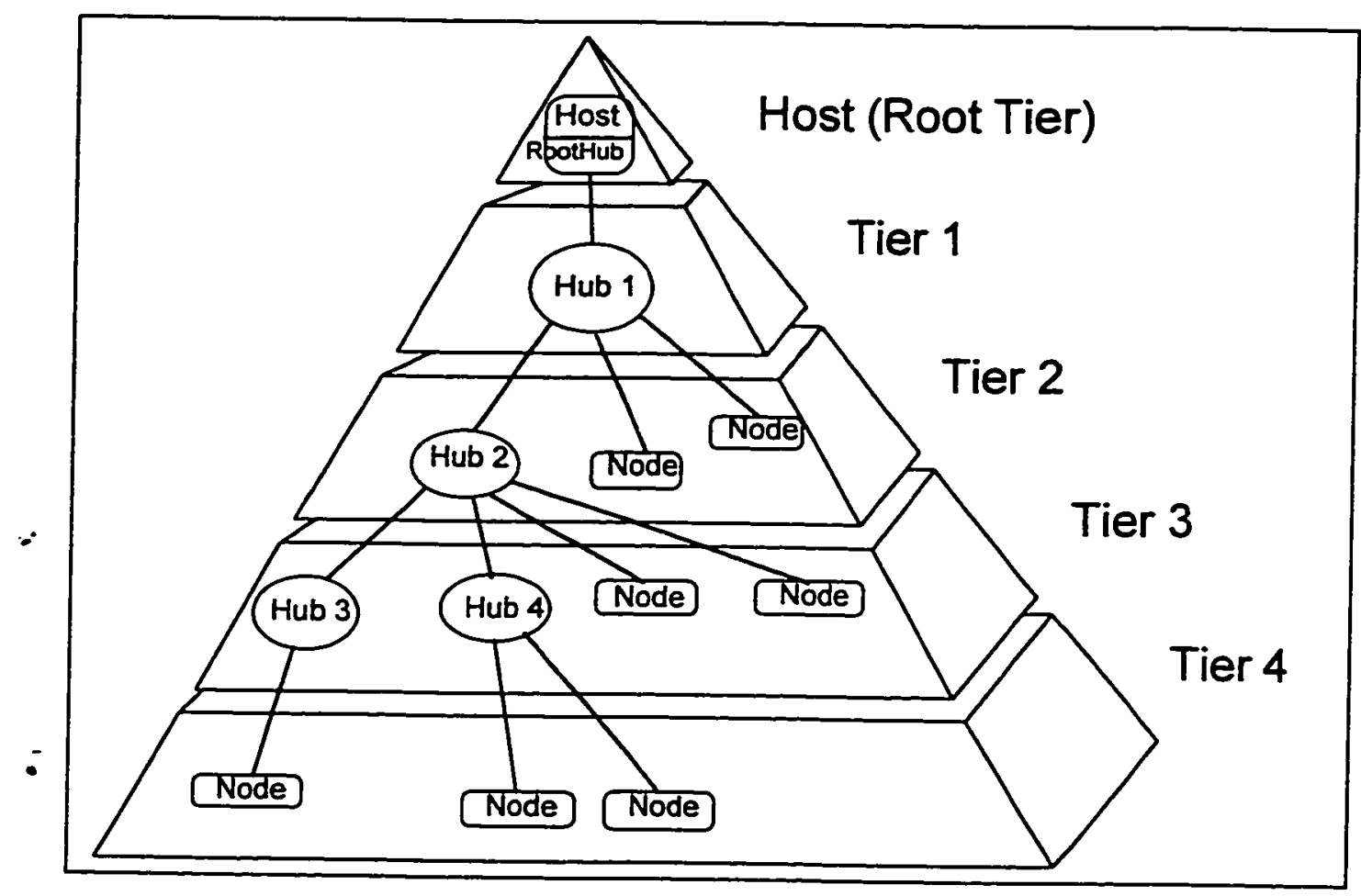

Figure 4.7: The USB bus topology [37]. 


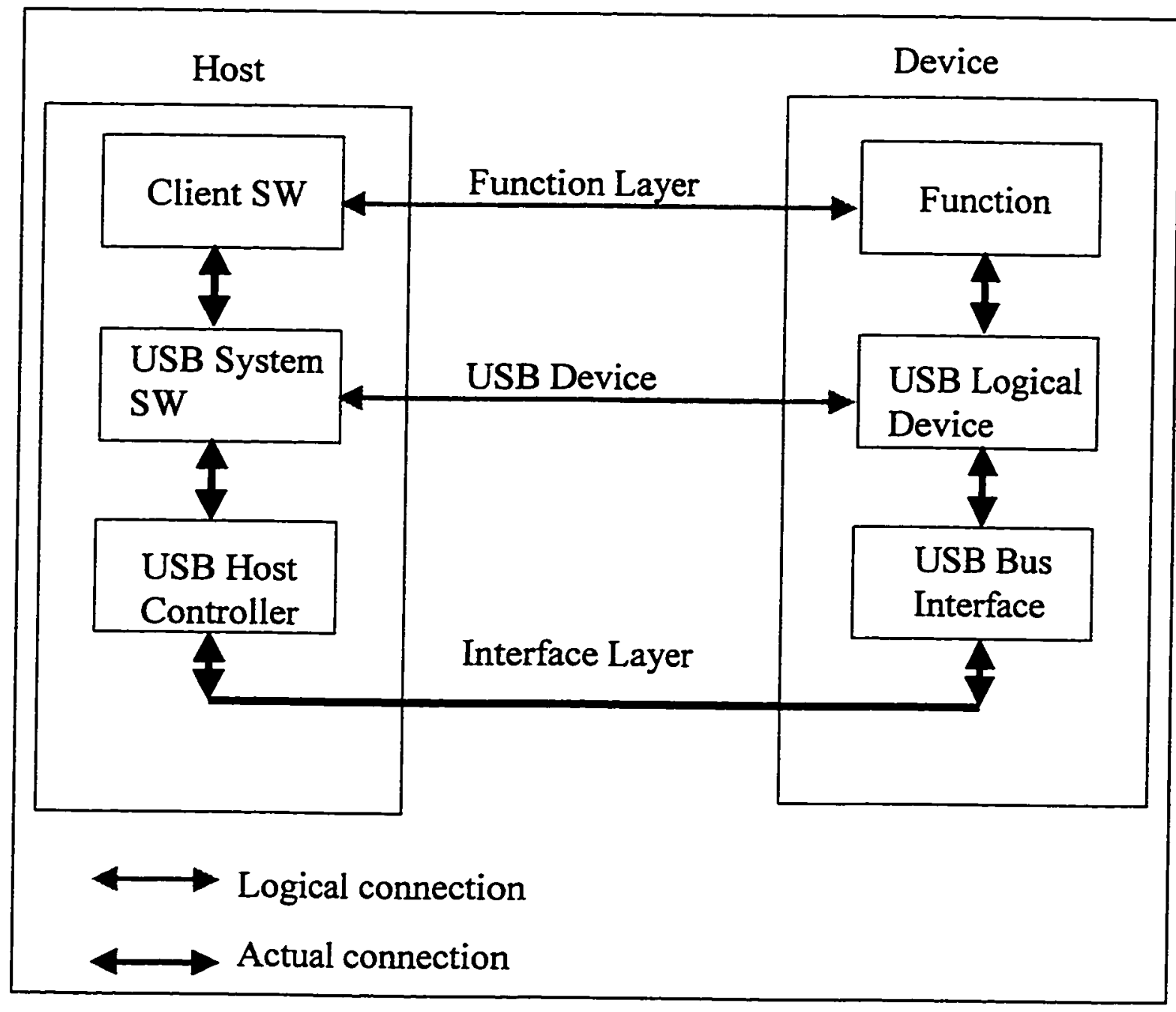

Figure 4.8: The USB's connection establishment between the device and the Host. 


\subsubsection{The Suggested CPU unit}

The CPU unit is limited to fewer instruction sets that are directly related to digital camera applications. From table 3.2, these instruction sets are the data transfer class, the arithmetic operation class, the logic class, and the system control class. These instruction set classes are mostly used in digital camera applications. It is the branch class that prevents most RISC core CPU from achieving a peak performance of one instruction per cycle throughput. By eliminating complex branch instructions and jump instructions, the suggested CPU unit will enhance its ideal peak throughput of one instruction per cycle.

On the other hand, memory caches will also improve the overall CPU performance. Currently, high-speed processors have between $32-\mathrm{KB}$ to $256-\mathrm{KB}$ cache to support frequently used instructions and data. For the interest of pricing and speed tradeoff, the suggested architectural design has a $32-\mathrm{KB}$ instruction cache, and a $64-\mathrm{KB}$ data cache. In addition, memory must be managed by a built-in memory management unit (MMU), which supports address translation, facilitates exception processing and manages different operating modes. Also, the MMU must support variable page sizes of 4-KB, $16-\mathrm{KB}, 64-\mathrm{KB}$ and $256-\mathrm{KB}$. This will give flexibility for the MMU to bring only the needed page. 


\subsection{The Performance}

\subsubsection{General Computational Capability}

The overall performances of the suggested architectural design consist of the performance of the CODEC unit, the CPU unit and the CPM unit. The overall performances must be fast enough to implement MPEG-2 image compression. In MPEG-2, the execution time for each process such as the MC, Q/IQ, VLC/VLD, and DCT/IDCT must be less than the 25 microseconds that was provided by ISO. Using two VDSP2 processors from Matsushinta Electric Industrial at $100 \mathrm{MHz}, \mathrm{MPEG}-2$ encoding can be accomplished less than 25 microseconds. Currently, other processors running at $250 \mathrm{MHz}$ to $300 \mathrm{MHz}$ also meet the MPEG-2 video timing. The suggested architecture should be able to achieve these timing requirements and beyond. Therefore, the overall performances of the architecture should be running at a maximum speed of $300 \mathrm{MHz}$ and minimum of $200 \mathrm{MHz}$ to keep up with technology trend.

\subsubsection{Potential for Big family of Signal Processing Functions}

Processors for printers, scanners, and image captures are similar to digital camera processors in that they all involve high-speed computation, high-speed I/O interface designs and perhaps image compression techniques. In many applications, a processor can be slightly modified to implement a product that was not originally designed for it. The suggested architectural design is considered an open system from these perspectives. The CPM includes high-speed and low-speed multimedia connections, standard UART 
connection and wireless infrared connections. Also, the CODEC unit equipped both JPEG and MPEG-2 compression standards to support a wide variety of products involving image compression. Last but not least, the CPU provides additional supports to configure the overall systems and control. With few modifications, the suggested architectural design has potential to become a big family of sign processing functions.

\subsection{Evaluation of the Need to Support Modern Operation Systems}

A big need in the electronic device industries is the capabilities to port from one platform to another. Currently, very few digital camera architectural designs come equipped with interface capabilities to work in Macintosh system, PC system, workstation, and peripheral devices such as printers, scanners, fax machine and copy machine. The CPM in the suggested architectural includes $1 / O$ interface designs to support all these systems. The USB feature allows interface to a PC as plug-and-plug. Also, it provides power to a digital camera or video camera that directly connects to a PC. This type of camera eliminated the need to supply power to the device separately. Furthermore, the IEEE 1394 allows connection to many network and device configurations. Lastly, the wireless infrared connection equips the suggested architecture to port images and data to printers, and scanners directly. 


\section{CHAPTER V}

\section{HDL SIMULATION OF XCAM-2000 CPU}

Commonly, a dedicated RISC type processor controls most major blocks of the camera system. In this chapter, we simulate a reduced version of the MIPS five-stage pipeline RISC CPU with 16-bit bus according to [8]. This 16-bit RISC CPU is named XCAM-2000. The XCAM-2000 is based on the MIPS R2000 instruction sets. Furthermore, it includes features that handles hardware and software hazards.

\subsection{MIPS Overview}

\subsubsection{Start-up Requirements}

The start-up operation for the XCAM-2000 CPU is such that a RESET signal must be asserted upon the power-up. The XCAM-2000 then clears all registers and checks for the LOAD signal. When this signal is asserted, it will enter the manual mode to allow program to be loaded into the instruction memory. This process continues until the LOAD is de-asserted. The XCAM-2000 CPU starts executing the program when both RESET and LOAD signals are de-asserted. 


\subsubsection{Instruction and Instruction Formats}

The XCAM-2000 CPU supports the instruction listed in table 5.1. It contains three types of instruction format: R-type, I-type and J-type. The instructions for R-type have three bits as opcode, six bits as the source register which contains two input sources with three bits in each input, three bits as the destination register, and four bits to decode each individual R-type instructions. The I-type is what many processors referred to as the immediate instructions. The I-type makes use of the 16-bit as follows: three bits for opcode, three bits for destination registers, and seven bits for offset address referencing. The J-type includes all jump instructions such as jump-and-link instruction (JAL), and jump instruction. The J-type makes use of the 16-bit as follows: three bits for opcode, and thirteen bits for offset address referencing. Figure 5.1 shows the details of each instruction type. 
(a) R-type format:

$\begin{array}{cllll}\text { Opcode } & \text { rs } & \text { rt } & \text { rd } & \text { function } \\ 3 & 3 & 3 & 3 & 4\end{array}$

$\begin{array}{lllll}3 & 3 & 3 & 3 & 4\end{array}$

(b) I-type format:

$\begin{array}{clll}\text { Opcode } & \text { rd } & \text { rt } & \text { offset } \\ 3 & 3 & 3 & 7\end{array}$

(c) J-type format:

Opcode offset

$3 \quad 13$

Figure 5.1: The three types of instruction format in the MIPS R2000 instruction sets. (a) Shows the R-type breakdown of the 16-bits. (b) Shuws the I-type breakdown of the 16bits. (c) Shows the J-type breakdown of the 16-bits. 


\begin{tabular}{|c|c|c|c|c|}
\hline Format & Opcode & Function & Mnemonic & Operation \\
\hline $\mathbf{R}$ & 000 & 0000 & Add & $R d=r S+r t$ \\
\hline $\mathbf{R}$ & & 0001 & Sub & $\mathrm{Rd}=\mathrm{rs}-\mathrm{rt}$ \\
\hline $\mathbf{R}$ & & 0010 & And & $\mathrm{Rd}=\mathrm{rs} \& \mathrm{rt}$ \\
\hline $\mathrm{R}$ & & 0011 & Or & \begin{tabular}{|l|}
$\mathrm{Rd}=\mathrm{rs} \mid \mathrm{rt}$ \\
\end{tabular} \\
\hline $\mathrm{R}$ & & 0100 & Inv & $\mathrm{Rd}=\mathrm{rs}$ \\
\hline $\bar{R}$ & & 0101 & Xor & $\mathrm{Rd}=\mathrm{rs}$ xor $\mathrm{rt}$ \\
\hline$\overline{\mathrm{R}}$ & & 0110 & SII & $\mathrm{Rd}=\mathrm{rs}<<1$ (logical shift left) \\
\hline $\mathbf{R}$ & & 0111 & Srl & Rd $=r s>>1$ (logical shift right) \\
\hline $\mathrm{R}$ & & 1000 & Sra & $\mathrm{Rd}=\mathrm{rS}>>1$ (arithmetic shift right) \\
\hline $\mathbf{R}$ & & 1001 & Slt & $\mathrm{Rd}=\mathrm{rs}<<1$ (arithmetic shift left) \\
\hline $\mathrm{R}$ & & 1010 & $\mathrm{~J}_{\mathbf{r}}$ & $\mathrm{Rd}=\mathrm{rs}$ (jump with return address) \\
\hline I & 001 & & Addi & $\mathrm{Rt}=\mathrm{rs}+$ offset $[7 . .0]$ (add immediate) \\
\hline $\mathrm{J}$ & 010 & & $\mathrm{~J}$ & $\begin{array}{l}\mathrm{PC}=(\mathrm{PC}[15 . .14] \text { concat } \\
\text { offset }[13 . .0])<<1 \\
\text { (unconditional jump) }\end{array}$ \\
\hline $\mathrm{J}$ & 011 & & Jal & $\begin{array}{l}\mathrm{R} 7=\mathrm{PC}+2 ; \\
\mathrm{PC}=(\mathrm{PC}[15 . .14] \text { concat } \\
\text { offset }[13 . .0])<<1 \\
\text { (jump and link) }\end{array}$ \\
\hline $\bar{I}$ & 100 & & Lw & $\mathrm{Rt}={ }^{*}$ (rs+ offset $[7 . .0]$ ) (load word) \\
\hline I & 101 & & Sw & ${ }^{*}(\mathrm{rs}+$ offset $[7 . .0])=\mathrm{rt}($ store word $)$ \\
\hline I & 110 & & Beq & If $(\mathrm{rs}=\mathrm{rt})$ then $\mathrm{pc}=\mathrm{pc}+$ offset $[7 . .0]+2$ \\
\hline I & 111 & & Bne & If $(\mathrm{rs} !=\mathrm{rt})$ then pc$=\mathrm{pc}+$ offset[7..0]+2 \\
\hline
\end{tabular}

Table 5.1: The instruction sets that support in the XCAM-2000 CPU simulation. These instruction sets are subset of the MIPS R2000 instructions. 


\subsection{The Top Level Design and Simulation}

The top level of the XCAM-2000 is showed in figure 5.2. The major blocks in this figure are the PC block, instruction memory block, control block, register file block, ALU block, data memory block, forward block, hazard detection block and the four pipeline blocks. All these blocks are interconnected to implement the XCAM-2000 CPU. Furthermore, these blocks are designed from very low level, which does not make use of any library parameter modules. They are based on the $\mathrm{D}$ flip-flop primitive only. The next section will describe the implementation of these blocks in-depth with details on the background theory and their basic building blocks. 


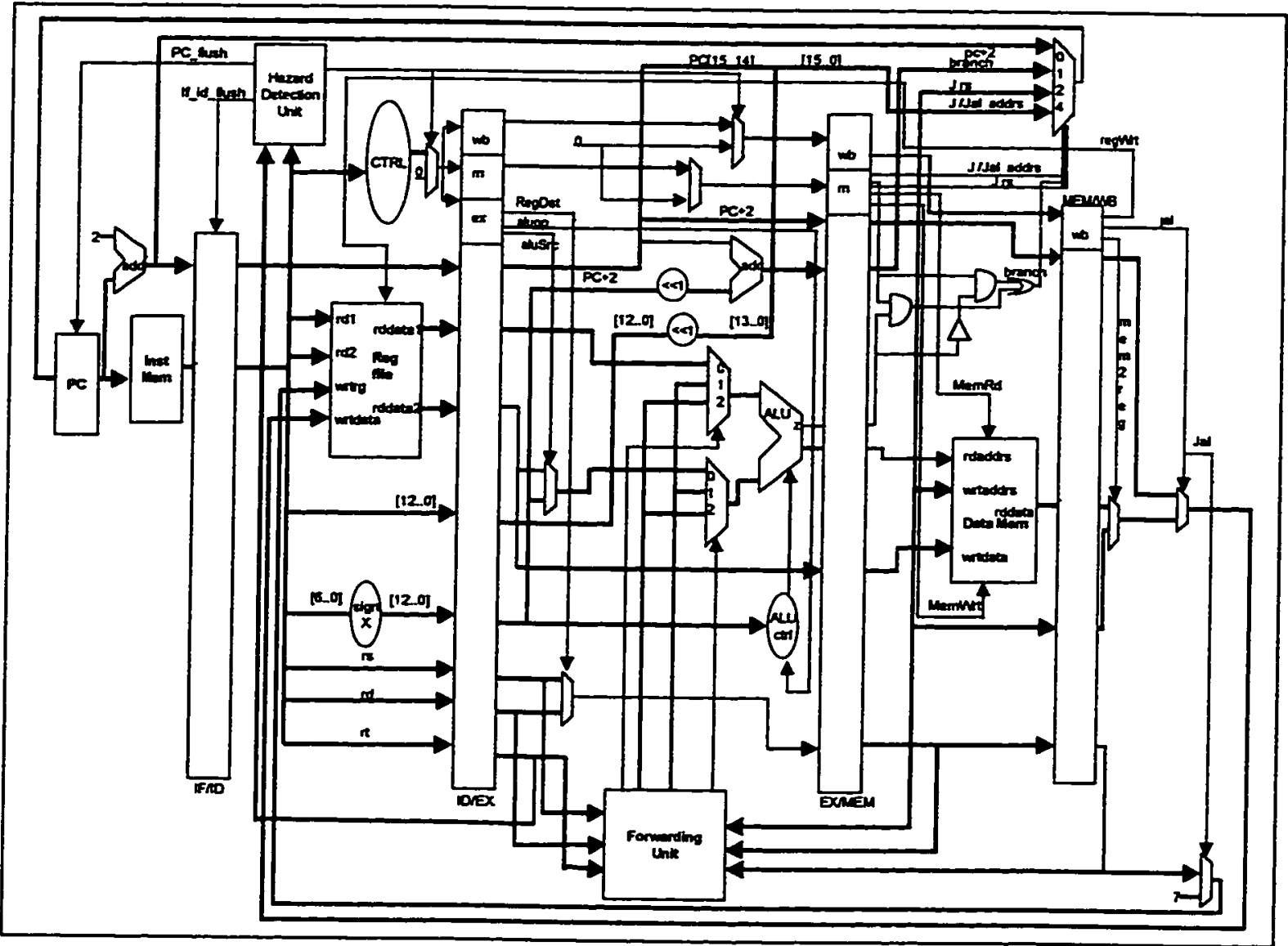

Figure 5.2: The interconnected of all the modules in the 16-bit CPU. The bold lines represent buses with two or more bits. The thin lines represent signals with only one bit. 


\subsection{The Subsystem and Basic Building Block Design}

\subsubsection{PC Module}

The program counter module handles the incremental of each instruction as the program is executed. The program counter content holds the address of the next instruction to be fetched from the instruction memory module. The design of the program counter module consists of sixteen D flip-flops connecting in parallel. Figure 5.3 shows the design of the program counter module. It has 15-bit data bus, a RESET signal, and a clock signal as input. The output is a 15-bit data bus.

\section{$\underline{\text { 5.3.2 Memory Module }}$}

There are two memory modules that are the same in design but being used as separate modules. These are the instruction memory module and the data memory module. The instruction memory module is used to store the instruction that will be fetched according to the address that is referenced in the program counter. On the other hand, the data memory stores the data that result from the register file module. Figure 5.4 shows the two memory modules are designed using fifteen $D$ flip-flops to form each register. The register is driven by the write signal and the write address bus. Upon appropriate address selection and with the write signal asserted, the data is latched into the register. The write address bus can be decoded up to $65 \mathrm{~K}$ address locations. We show only thirty-one address locations to illustrate the design. To read data from memory, only the read signal and the read address bus are required. Upon appropriate selection of the read address and with the read signal asserted, the output of the memory 
module will contain the content of the register output.

\subsubsection{Pipelines}

There are four pipelines that are used to separate the data path into five stages of execution. The design of these four pipelines is the same. Basically, these pipelines are $\mathrm{D}$ registers that hold the data in its content. Upon clocking, the data will be latched into the $\mathrm{D}$ register and therefore becomes available to be used in the next stage. The pipeline design is a strategy to allow the data path of CPU design to execute in stages which allows each stage to act as an independent unit. The overall result of the pipeline is a peak performance of one instruction per clock cycle throughput.

\subsubsection{Register file Module}

The register file design is similar to the memory module design. It consists of two inputs to read the register content into source operand to perform an operator. A write to the register file consists of the write data bus and the write adidress bus that specifies to which register to write the data to. The design-of the register file is based on the D flipflops connecting in parallel to form a register. The register input is the write data bus content. The write address will be decoded to enable the $\mathrm{D}$ flip-flop register to latch the input into the register. The MUX design with the read data bus allows the output of the register to be selected as the output. Figure 5.5 shows the design. 


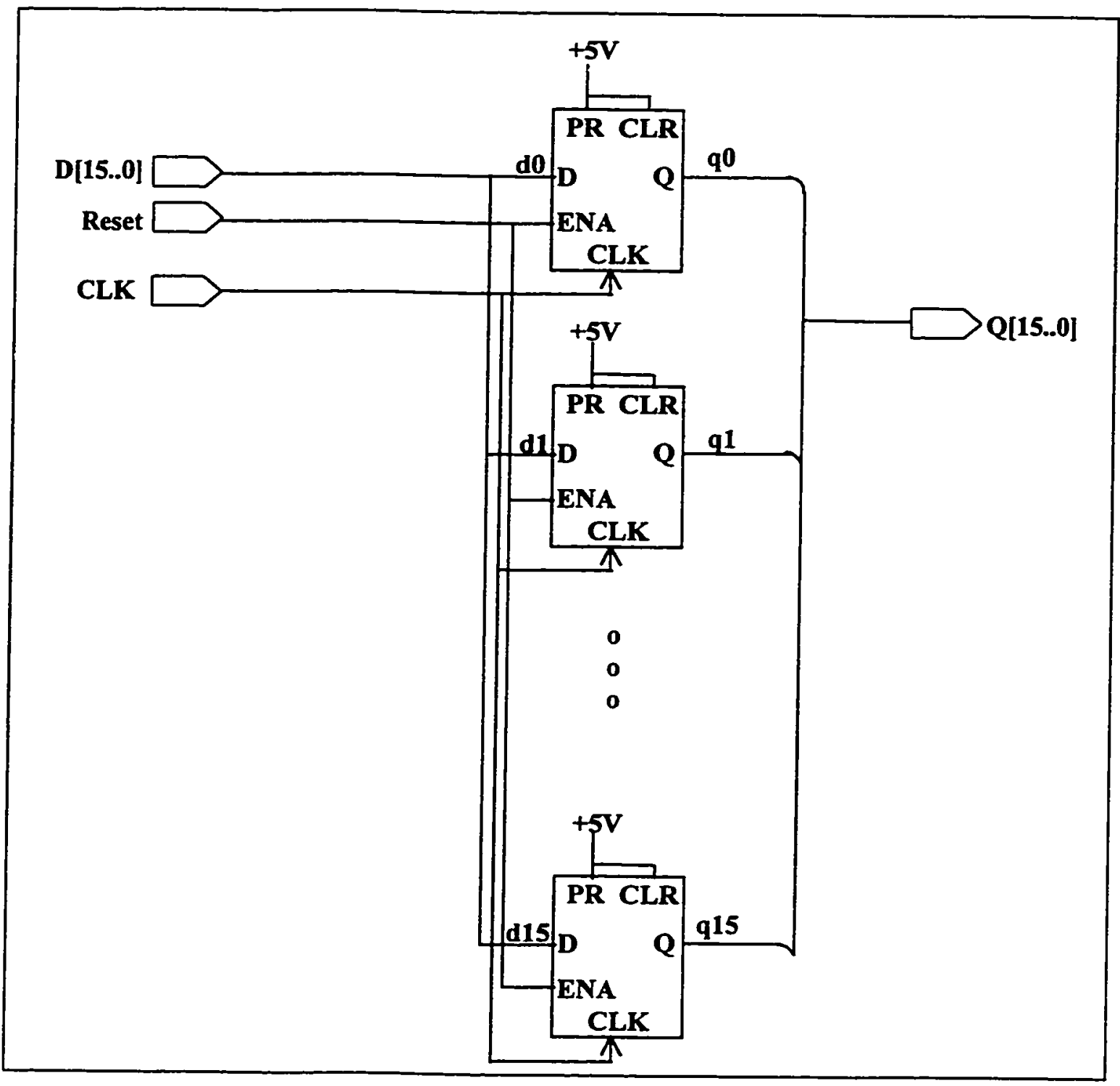

Figures 5.3: Design of the program counter. It uses sixteen D flip-flops connecting in parallel. Upon clocking, the data will be latched into the register. Reset signal will clear the content of the 16 registers. 


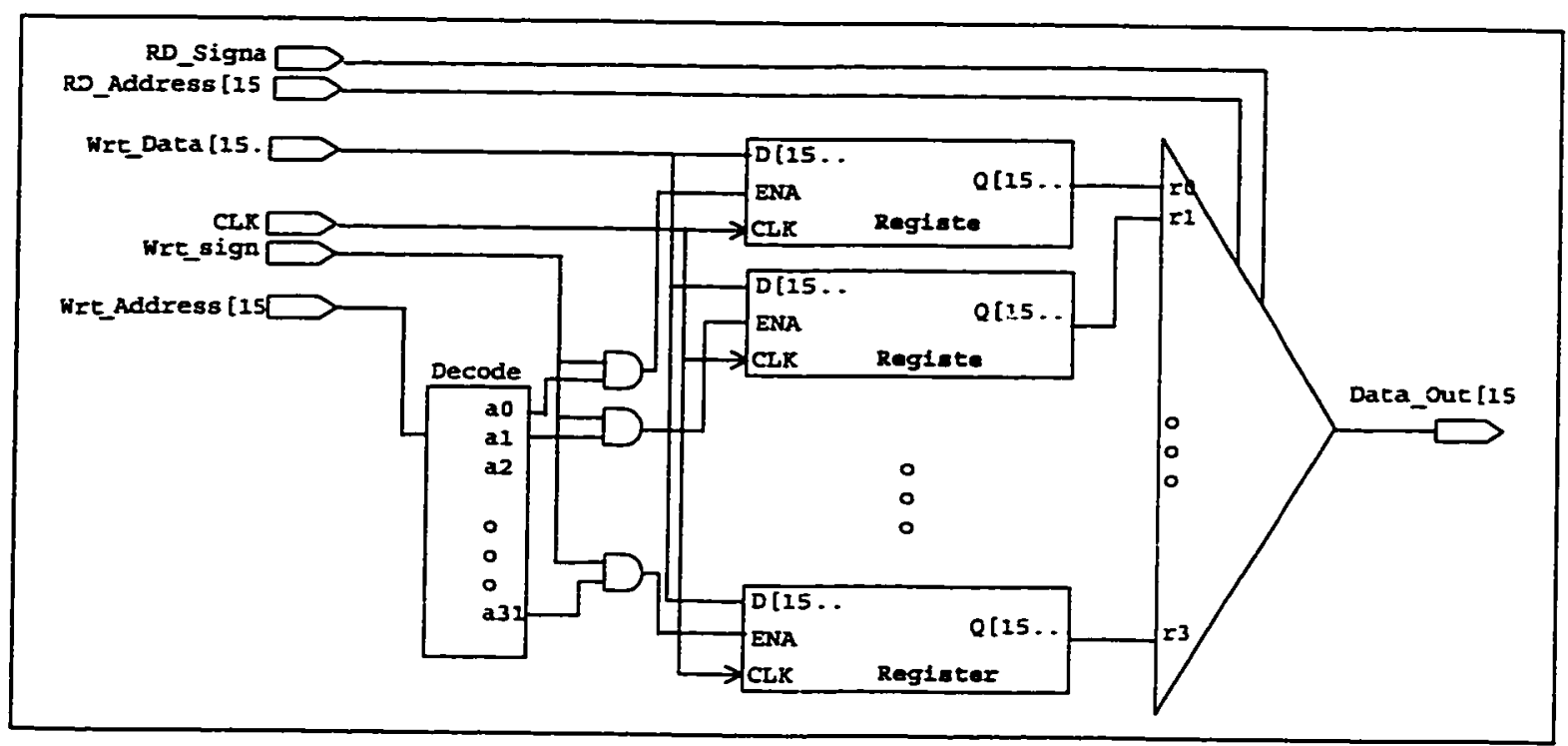

Figure 5.4: Design of the Memory module. Each register is designed from sixteen D flipflops connecting in parallel. For illustration purposes, the design has only thirty-one registers. Ideally, it can decode up to $65 \mathrm{~K}$ address locations. The RD_Address bus and the RD_signal signal are both used to select the data from register to be put out. 


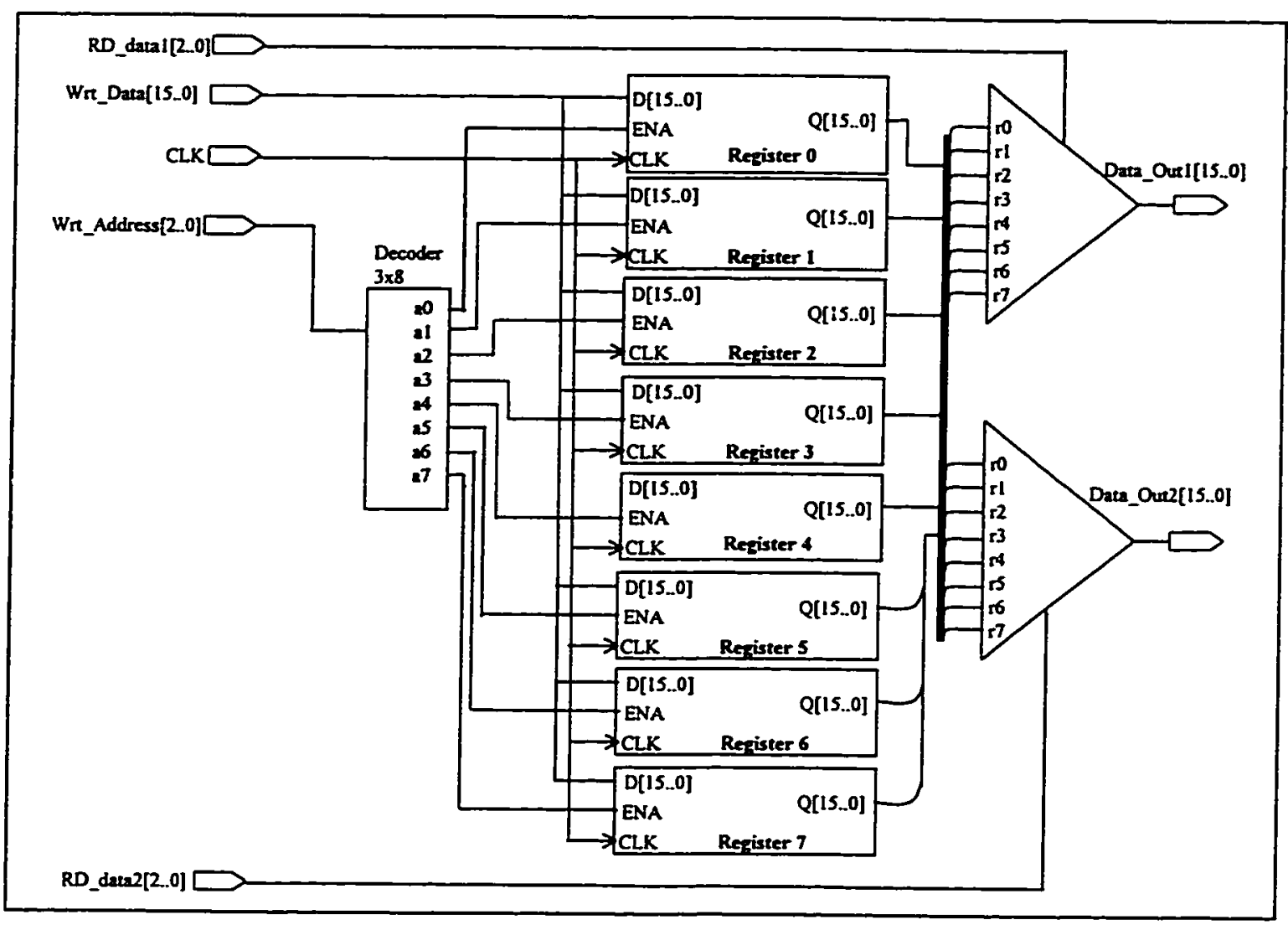

Figure 5.5: The design of the register file. It consists of seven registers with $D$ flip-flop connecting in parallel. The write address will decode which register to write to. The read of DATA 1 and DATA 2 is a multiplexer (MUX) design that makes selection of which register to read from. 


\subsubsection{6-bit ALU Module}

The ALU Module is a 16-bit input of two source operands and an ALU selection for the operator. Figure 5.6 shows the design of the ALU module. The ALU Control Module that uses the opcode and the first six least significant bits of the instruction word generates the ALU selection. Table 5.2 shows the ALU selection, its corresponding function and the result. The design of the ALU module is to accommodate these functions and produce their results. Based on the ALU selection, the individual function is implemented accordingly. The ADD and SUB operator are implemented using four 4bit adders connecting in parallel. The logical shift operators are just shifting the bits left or right depending on the operator. The status generator generates the conditional status flag, which bases on the result of the function it just computed and the two source operand. For example, the CARRY flag is the carry out of the fourth adder. The ZERO flag is determined by the following equation:

(5.1) ZERO $=$ ! $(F[15] \# F[14] \# F[13] \# F[12] \# F[11] \# F[10] \# F[9] \# F[8] \# F[7] \# F[6]$ \# $F[5] \# F[4] \# F[3] \# F[2] \# F[1] \# F[0])$

Where $!=$ NOT and $\#=O R$.

The NEGATIVE flag is the most significant bit of the result which is bit $15^{\text {th }}$. The OVERFLOW flag uses the two source operands and the result to check for any overflow occurred. The condition for the OVERFLOW flag be set is such that if the two sources 
are both negative numbers and the result is positive, than the overflow has occurred, or if the two sources are both positive numbers and the result is negative, then overflow has occurred as result of the operation. Otherwise, no overflow has occurred.

\begin{tabular}{|c|c|c|}
\hline ALU Selection & Function & Result \\
\hline 0 & ADD & F and Status Flag \\
\hline 1 & SUB & F and Status Flag \\
\hline 2 & AND & F and Status Flag \\
\hline 3 & OR & F and Status Flag \\
\hline 4 & NOT EQ & F and Status Flag \\
\hline 5 & XOR & F and Status Flag \\
\hline 6 & SLL & F and Status Flag \\
\hline 7 & SRL & F and Status Flag \\
\hline 8 & SRA & F and Status Flag \\
\hline 9 & SLT & F and Status Flag \\
\hline 10 & JR & Content of \$7 \\
\hline
\end{tabular}

Table 5.2: The functions implemented in the ALU Module of the XCAM-2000. 


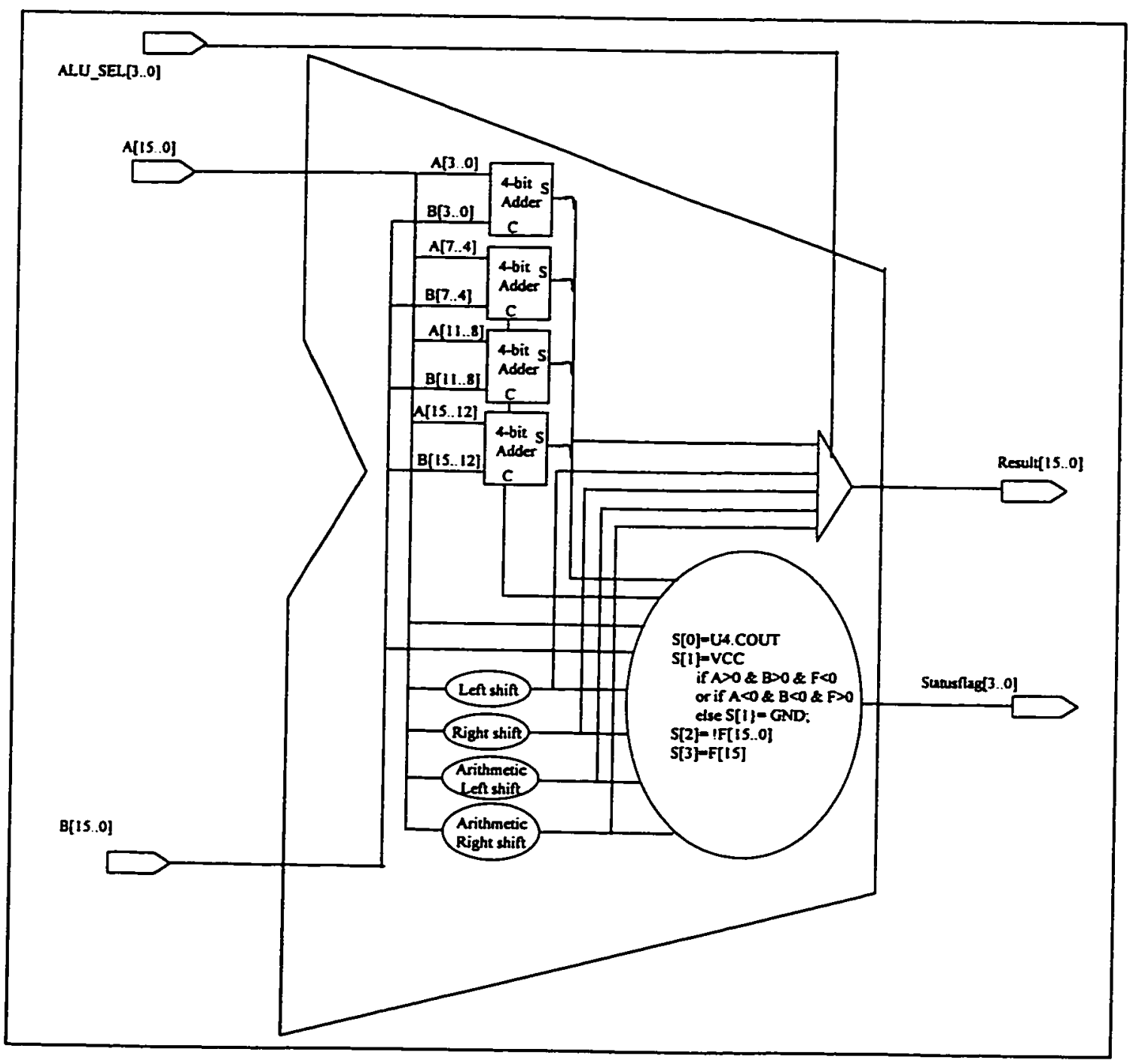

Figure 5.6: ALU Design. The status flag is generated by using both input A, B, and the result of the function 


\subsubsection{Control Module}

The Control Module generates all the control signals to be used for all the main blocks in the top-level design. These signals are listed in table 5.3 with the different stages that the signal is actually active. The implementation of this table is a state machine. Figure 5.7 shows the control state machine. It has three states. The first state is the reset state. It checks for RESET signal to be asserted. If RESET signal is not asserted, than the next state is the Load State. In the Load State, it checks for LOAD signal and proceeds to state S0. In the S0 state, the opcode is used to check on the type of instruction. Based on the opcode, the appropriate control signal is active as specified in table 5.3.

\begin{tabular}{|c|c|c|c|c|c|c|c|c|c|c|c|c|}
\hline \multirow[t]{2}{*}{$\begin{array}{l}\text { Instruction } \\
\text { Type }\end{array}$} & \multicolumn{4}{|c|}{$\begin{array}{l}\text { Execution Stage } \\
\text { Control Lines }\end{array}$} & \multicolumn{4}{|c|}{$\begin{array}{l}\text { Memory Stage } \\
\text { Control Lines }\end{array}$} & \multicolumn{2}{|c|}{$\begin{array}{c}\text { Write Back } \\
\text { Stage Control } \\
\text { Lines }\end{array}$} & \multicolumn{2}{|c|}{$\begin{array}{c}\text { Jump } \\
\text { Control } \\
\text { Lines }\end{array}$} \\
\hline & $\begin{array}{l}\text { Req } \\
\text { Dst }\end{array}$ & $\begin{array}{l}\text { ALU } \\
\text { Op1 }\end{array}$ & $\begin{array}{l}\text { ALU } \\
\text { Op0 }\end{array}$ & $\begin{array}{l}\text { ALU } \\
\text { SrC }\end{array}$ & Beq & Bne & $\begin{array}{l}\text { Mem } \\
\text { Read }\end{array}$ & $\begin{array}{l}\text { Mem } \\
\text { Wrt }\end{array}$ & $\begin{array}{l}\text { Reg } \\
\text { Wr }\end{array}$ & $\begin{array}{c}\text { MemTo } \\
\text { Reg }\end{array}$ & \begin{tabular}{|l|l|}
$\mathrm{J}$ & $\mathrm{Jal}$ \\
\end{tabular} & \begin{tabular}{|c|}
$\mathrm{J}$ \\
$\mathrm{J}$
\end{tabular} \\
\hline R-Type & 1 & 1 & 0 & 0 & 0 & 0 & 0 & 0 & 1 & 0 & \begin{tabular}{|l|l}
$x$ & $x$ \\
\end{tabular} & $\mathrm{X}$ \\
\hline $\mathrm{LW}$ & 0 & $\overline{0}$ & 0 & 1 & 0 & 0 & $T$ & 0 & 1 & I & \begin{tabular}{|l|l|}
$\mathrm{X}$ & $\mathrm{X}$ \\
\end{tabular} & $\mathrm{X}$ \\
\hline SW & $\mathrm{X}$ & 0 & 0 & 1 & 0 & 0 & 0 & I & 0 & $\bar{X}$ & \begin{tabular}{|l|l}
$X$ & $X$ \\
\end{tabular} & $\mathrm{X}$ \\
\hline Beg & $\bar{X}$ & 0 & 1 & 0 & $I$ & 0 & 0 & 0 & 0 & $\mathrm{x}$ & \begin{tabular}{|l|l}
$\mathrm{X}$ & $\mathrm{X}$ \\
\end{tabular} & $\mathrm{x}$ \\
\hline Bne & $\bar{x}$ & 0 & 1 & 0 & 0 & 1 & 0 & 0 & 0 & $\mathrm{X}$ & \begin{tabular}{|l|l|}
$\mathrm{X}$ & $\mathrm{X}$ \\
\end{tabular} & $x$ \\
\hline$J$ & $\bar{x}$ & $\bar{X}$ & $\bar{X}$ & $\mathrm{X}$ & $\bar{x}$ & $\bar{x}$ & $\bar{X}$ & $\bar{X}$ & $\bar{x}$ & $\bar{X}$ & \begin{tabular}{|l|l|}
1 & $X$ \\
\end{tabular} & $x$ \\
\hline $\mathrm{Jal}$ & $\bar{x}$ & $\bar{x}$ & $\bar{x}$ & $\bar{x}$ & $\bar{X}$ & $\bar{x}$ & $\bar{x}$ & $\bar{x}$ & $\mathrm{X}$ & $\mathrm{X}$ & $\mid \begin{array}{ll}\mid x & 1\end{array}$ & $x$ \\
\hline $\mathbf{J r}$ & $\bar{X}$ & $\bar{x}$ & $x$ & $\bar{X}$ & $\bar{X}$ & $\bar{X}$ & $\bar{X}$ & $\bar{X}$ & $\bar{x}$ & $\bar{X}$ & \begin{tabular}{|l|l|}
$x$ & $x$ \\
\end{tabular} & 1 \\
\hline
\end{tabular}

Table 5.3: The control signals that are used in each stage of the pipeline along with their type of instruction. 


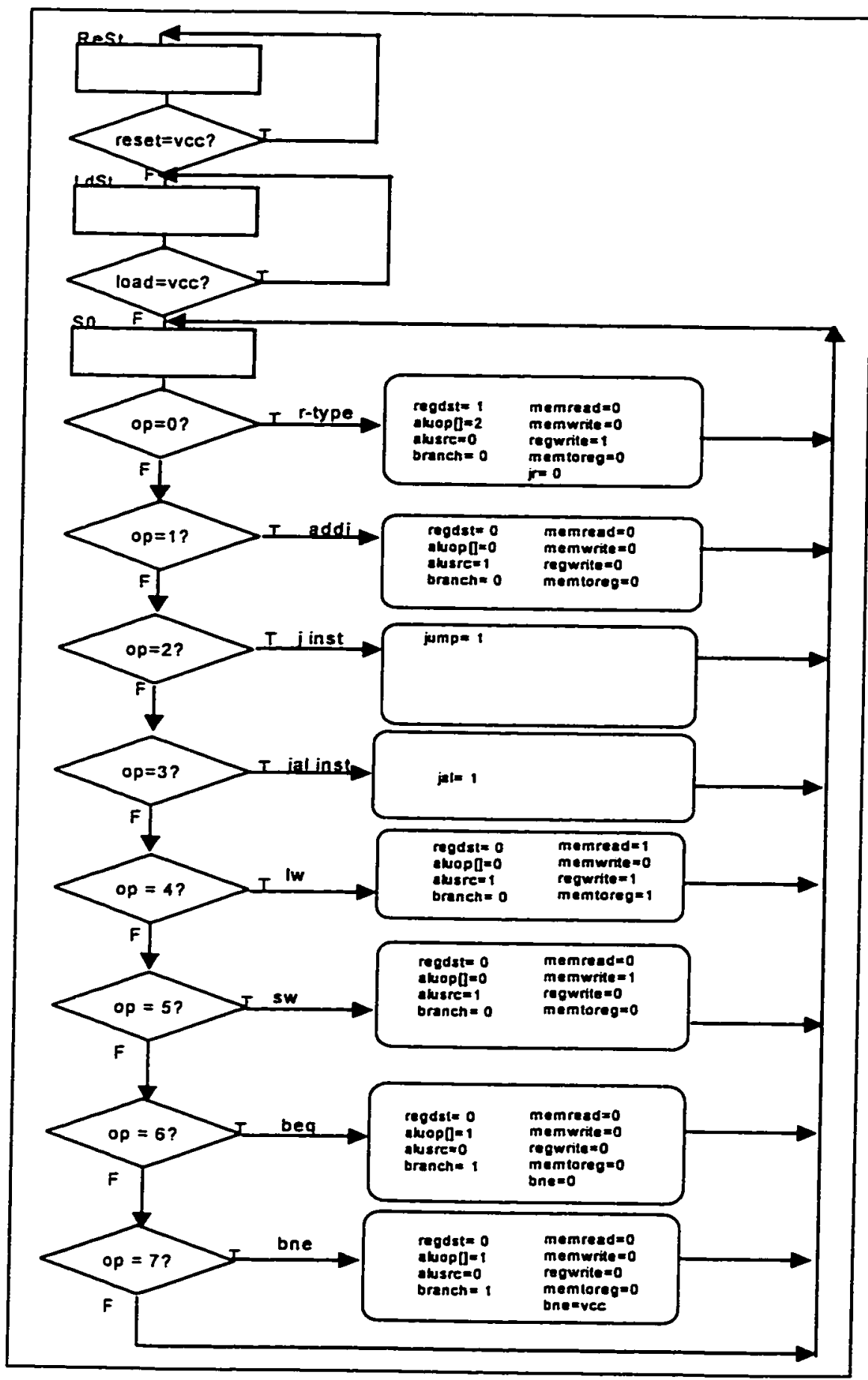

Figure 5.7: The state machine to generate all the control signals in the XCAM-2000 CPU. In this diagram, VCC and "1" both mean logic high. 


\subsubsection{Hazard Modules}

There are three types of hazards that must be resolved when designing a multiple stage RISC CPU: structural hazards, data hazards and control hazards. Structural hazards occur when two instructions try to write to the register file at the same time. Figure 5.8 illustrates this point. The load word ( $\mathrm{LW}$ ) instruction needs to access memory thus it has five stages. The R-type instruction does not need to access memory thus it only has four stages. At the write stage, both instructions try to write to the same register file at the same time.

On the other hand, data hazards are similar to structural hazards but the cause of the hazard is due to data. Figure 5.9 shows the order in which the instructions are executed. The $\mathrm{rl}$ in the ADD instructions is needed as the source operand for the SUB, $\mathrm{AND}, \mathrm{OR}$, and the XOR instructions. Well, the $\mathrm{rl}$ content has not been written to memory thus these instructions can not use it. Thus, this is a data hazard. Data hazard occurs when the content of a destination register in an instruction is needed as the source operand for the next following four instructions. There are several ways to correct the data hazards. One way is to use a smart compiler to reschedule the order of the instruction to eliminate the dependant of the data source. Another way is to forward the content of the destination register as soon as it is available. This method is a viable solution and we implemented this solution to resolve hazards in the XCAM-2000. 


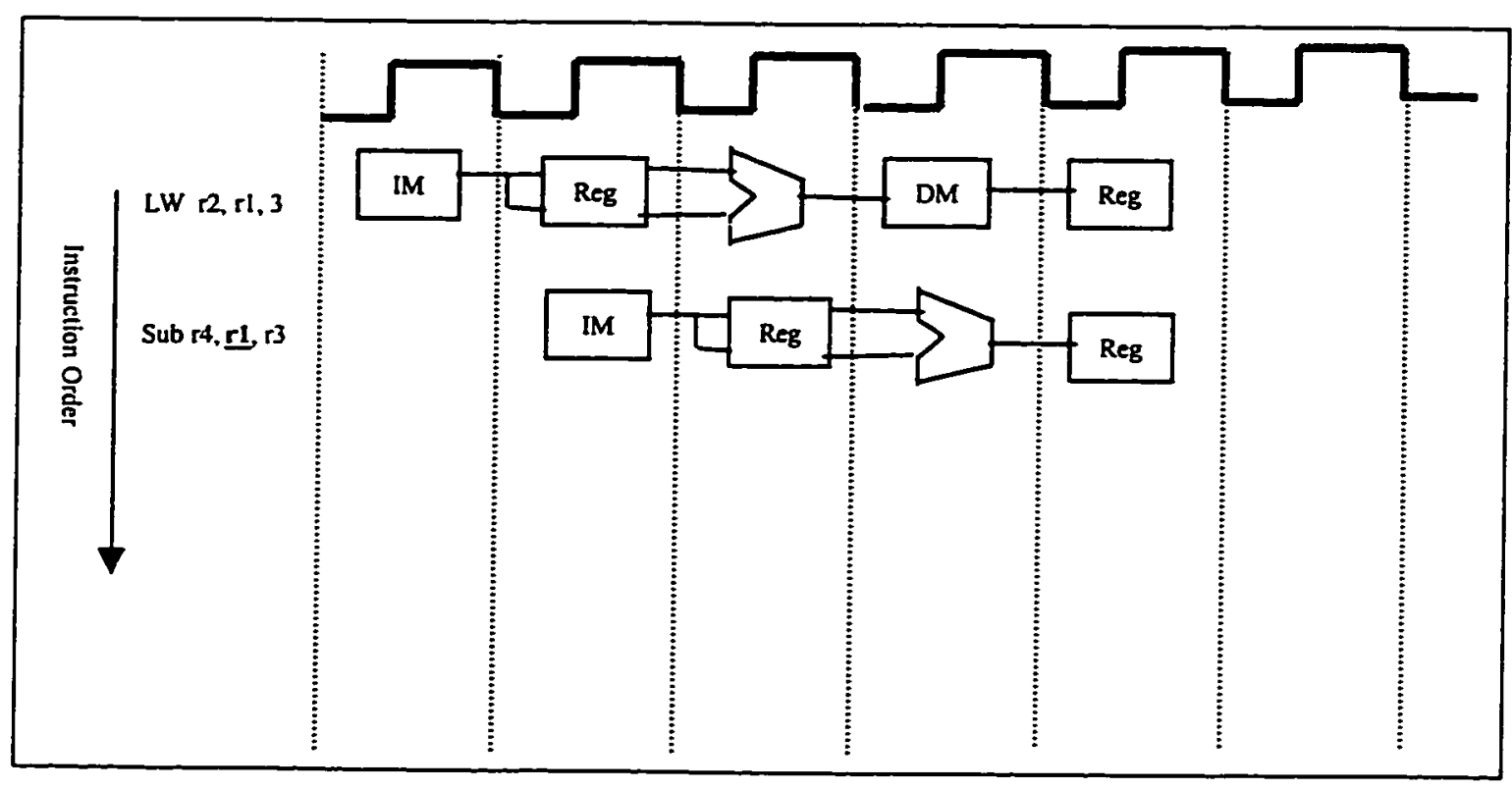

Figure 5.8: Structural hazards occur when LW or SW instruction and R-type instruction try to write to the same location at the same time. The LW or SW instruction reference memory so it has 5 stages. The R-type instruction does not need to reference memory so it has 4 stages. When it tries to write to the same location at the same time on the write stage, hazard occurs. 


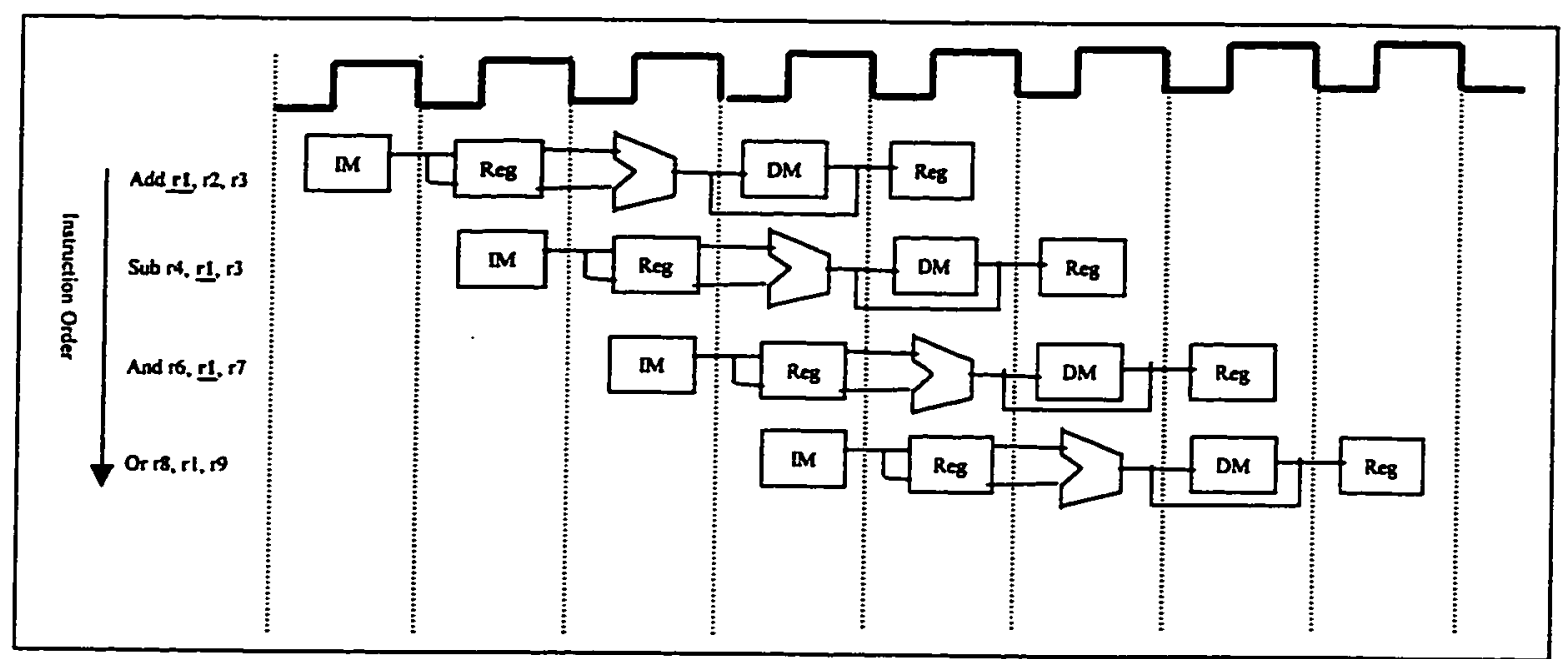

Figure 5.9: Data hazards result when the register content is not written by the first instruction but is to be used by the following four instructions. The $\mathrm{rl}$ register content in the ADD instruction has not been written yet but its content is needed by the SUB, AND, and the OR instructions. 
Last but not least, the control hazards occur when a conditional branch or jump instruction is taken. Recall, the way pipeline works is that in every clock cycle, information passes from one stage to the next and new information gets loaded into the first stage. Well, in the case of conditional branch and jump instructions, the decision whether to branch or jump is not yet determined until the ALU stage. However, by the time the decision is made at the ALU stage, two instructions have been fetched into the pipeline. These-instructions must not be moved to the next stages. The solution to resolve all of these three hazards involve a combination of inserting bubble and also a forwarding unit, which can detect hazards and forward data to the appropriate stage.

\subsection{The Evaluations}

Table 5.4 shows the instructions to be tested and its corresponding result. The waveform in figure 5.10 reflect table 5.4. The ADDI instruction adds the content of register $\$ 0$ to 3 and store the result in register $\$ 1$. The second ADDI instruction adds the content of $\$ 1$, which has the value 3 , to 2 and store the result to register $\$ 2$. These two instructions emphasize that the hazard detection and forwarding units works correctly.

Table 5.5 shows the test case for the LW and SW instructions. This test case shows that the first SW instruction is writing to register $\$ 3$ with the result of 6 . The LW instruction retrieves the same memory location that the SW instruction wrote to and the retrieve data is indeed a 6. Figure 5.11 reflect this test case. 


\begin{tabular}{|l|l|l|}
\hline Address & Instruction & Hex Code \\
\hline 0004 & ADDI \$1, \$0,3 & 2083 \\
\hline 0006 & ADDI \$2, \$1,2 & 2502 \\
\hline 0008 & ADD \$3, \$2, \$1 & $08 \mathrm{~B} 0$ \\
\hline $000 \mathrm{~A}$ & SUB $\$ 4, \$ 3, \$ 2$ & $0 \mathrm{D} 41$ \\
\hline $000 \mathrm{C}$ & AND \$5, \$4, \$2 & 1152 \\
\hline $000 \mathrm{E}$ & JR \$7 & $1 \mathrm{COA}$ \\
\hline
\end{tabular}

Table 5.4: The test case for various instructions in the XCAM-200 CPU.

\begin{tabular}{|l|l|l|}
\hline Address & Instruction & Hex Code \\
\hline 0004 & ADDI \$1, \$0,2 & 2082 \\
\hline 0006 & ADDI \$3, \$1,4 & 2584 \\
\hline 0008 & SW \$3, \$1, 1 & A581 \\
\hline $000 \mathrm{~A}$ & ADDI $\$ 0, \$ 0,0$ & 2000 \\
\hline $000 \mathrm{C}$ & LW $\$ 4, \$ 1,1$ & 8601 \\
\hline $000 \mathrm{E}$ & AND $\$ 2, \$ 1, \$ 4$ & 0622 \\
\hline 0010 & SW $\$ 4, \$ 3,9$ & AE09 \\
\hline 0012 & JR $\$ 7$ & 1C0A \\
\hline
\end{tabular}

Table 5.5: The test case for LW and SW instructions in XCAM-2000 CPU. The first $\mathrm{SW}$ store 6 into memory location 3 . The LW reads 6 from the same memory. 


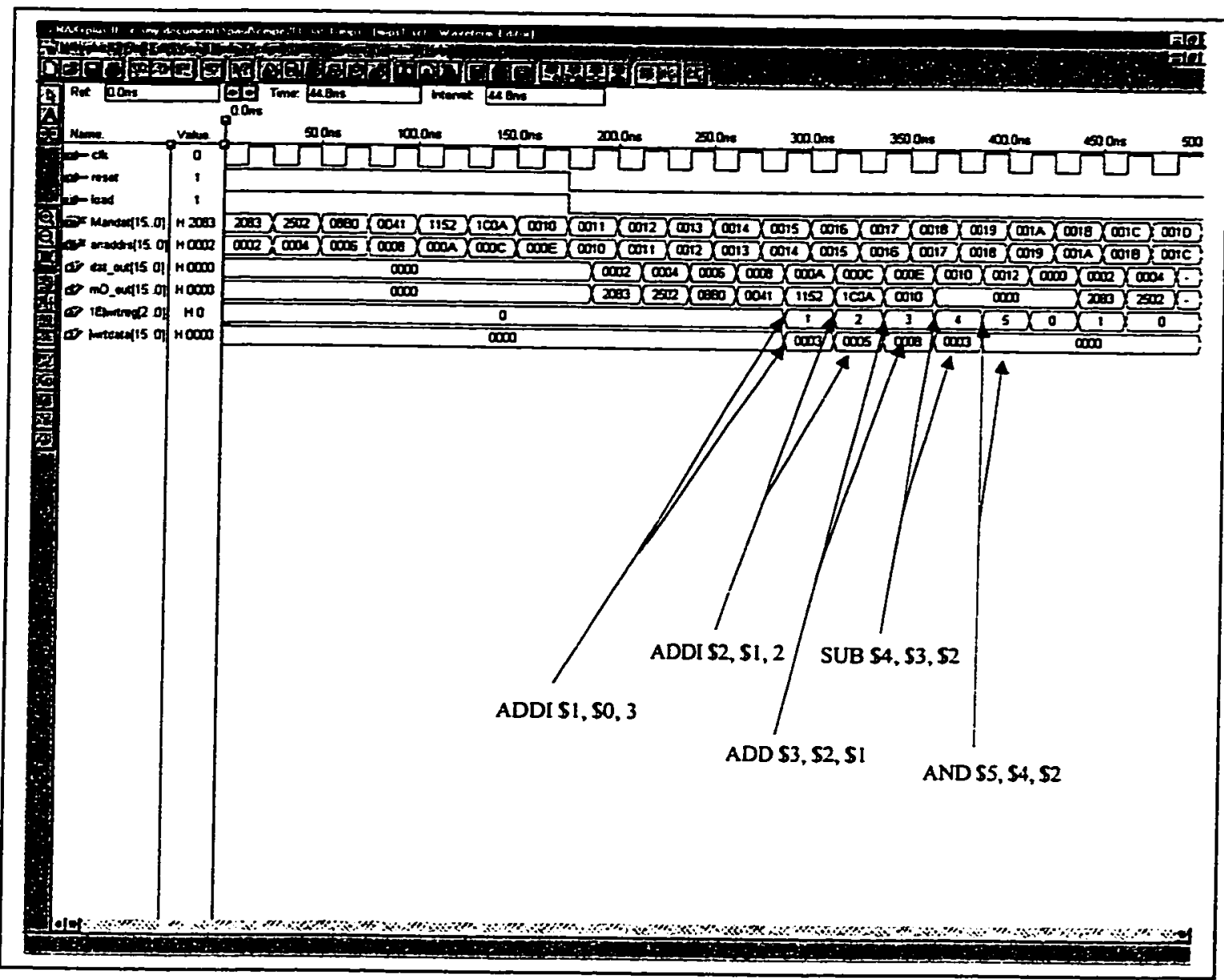

Figure 5.10: Waveform for various instructions. This waveform reflects the instructions in table 5.4 


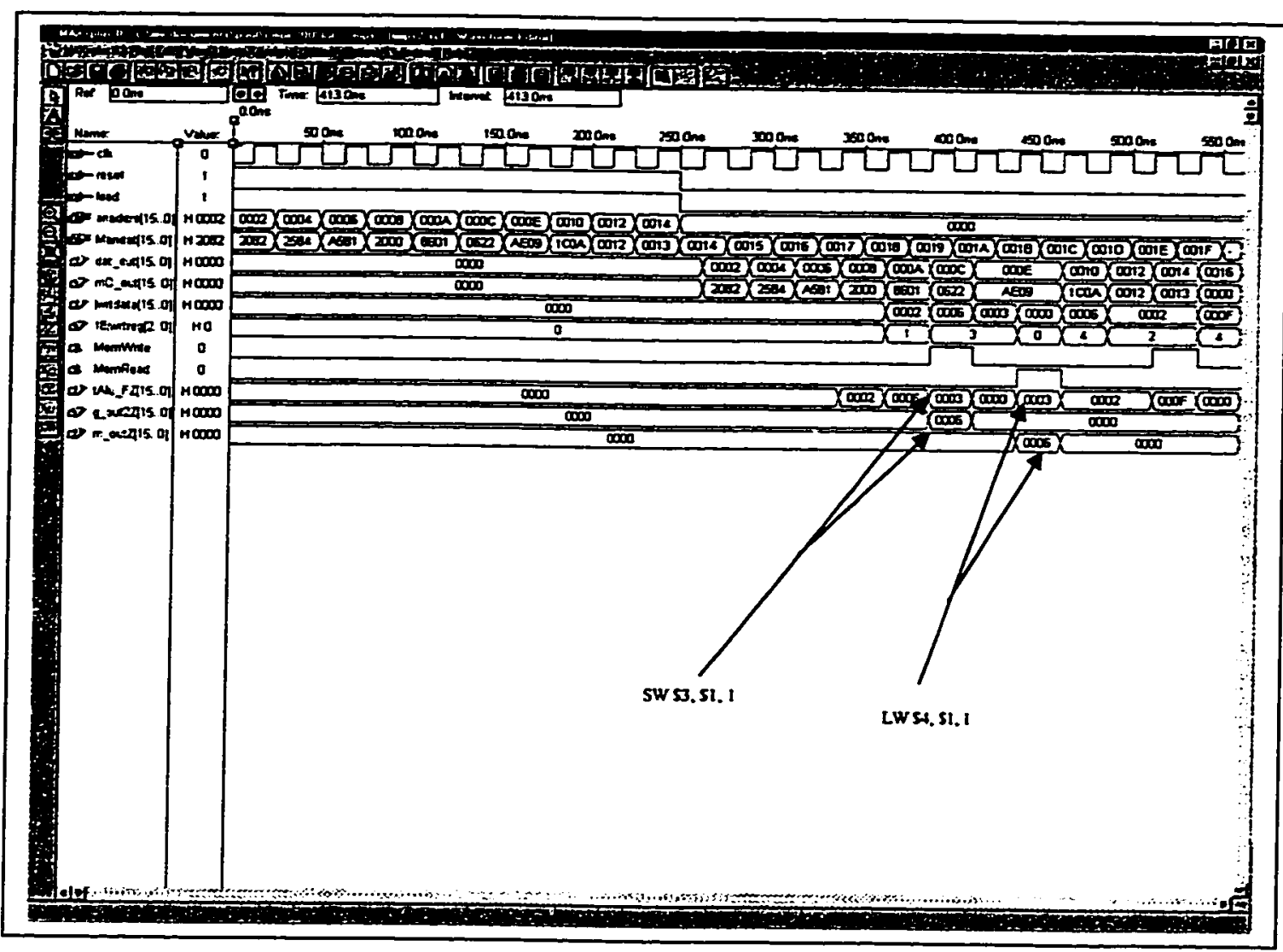

Figure 5.11: Waveform for the $\mathrm{LW}$ and $\mathrm{SW}$ instructions. This waveform reflects the instructions in table 5.5 


\section{CHAPTER VI}

\section{CONCLUSION}

The purpose of this research was to evaluate the architectural design for digital camera applications. The core technologies for digital camera applications include (1) CCD sensor, (2) image compression technique, and (3) combination of both RISC core and DSP core to serve as the CPU.

A CCD sensor is the front-end unit that performs color image sensing functions. Due to the advancement in semiconductor technology, even present low-end digital cameras are equipped with a color CCD sensor up to one mega-pixel resolution and relatively low cost. This study has revealed the fact that most of the digital cameras today have compression capability. JPEG is the primary compression standard. However, MPEG-2 standard is becoming more popular. MPEG-2 allows greater input formats, and capability to deal with both motion video and still images.

In this thesis, we emphasized the investigation and evaluation of the enabling technology, namely, the CPU for image processing and compression applications. We have carefully examined the most representative designs in this field, analyzed the architectural designs and their instruction sets. This study indicates that current CPU designs include capability to implement image CODEC, and wide variety of low speed and high speed I/O interface functions. Namely, these I/O designs are SPI, SCI, SMC, $I^{2} \mathrm{C}$, wireless infared, and USB. 
We suggested an architectural design that includes more specific design functions for digital camera applications. These design functions include the LCD display for instant viewing, $\mathrm{I} / \mathrm{O}$ interface design that includes multimedia communication such as IEEE 1394, and a CPU with more specific instructions for data movement.

We have simulated a reduced MIPS R2000 instruction CPU, called XCAM-2000 according to the description in [8]. The XCAM-2000 CPU is a five stages pipeline processor with an ideal peak execution of one instruction per cycle throughput. The intention for the simulation is to illustrate the RISC core characteristics such as pipeline stages and basic hazards to achieve an ideal peak execution of one instruction per cycle throughput. It does not reflect the overall RISC core CPU design. Also, due to constraints in time and resource allocation, not every instruction is fully simulated. To fully implement this simulation will require more specification on the instructions, more logic designs, all analog designs, and other L/O designs to serve as a complete CPU design.

The evaluation of an architectural design for digital camera application is the first of its kind. Future evaluation will be focused on system-on-a-chip in which all the major modules are shrunk onto one-chip for cost effectiveness. Also, future direction for the XCAM-2000 will need to include: (1) a MAC design for fast image processing, (2) a full implementation of all the necessary instructions, and (3) a complete simulation of the instructions before a netlist can be generated and downloaded into a field programmable gate array (FPGA) for testing. 


\section{REFERENCES}

[1] The Illustrated Science and Invention Encyclopedia, Westport, H.S. Stuttman Inc., pp. 425-435, 1974.

[2] S. Boyle and G. E. Smith, "Charge Coupled Semiconductor Devices," Bell Systems Technical Journal, vol. 49, pp. 587-593 (1970).

[3] B. E. Bayer, US patent \#3,971,965 (1976).

[4] J. Janesick, G. Williams, and M. Blouke, "High Speed Scientific CCDs," CCD Conference on the Grand Cayman Island hosted by Bonner Denton (University of Arizona) December 1995.

[5] J. Janeick, T. Elliott, R. Winzeread, J. Pinter, and R. Dyck, "Sandbox CCDs," in Charge-coupled Devices and Solid Optical Sensors V, M. M. Bloke, ed., SPIE Proceedings vol. 2415, pp. 2-42, 1995.

[6] J. Janesick, "CCDs: The Inside Story," CCD Astronomy, Winter 1997, pp. 9-15.

[7] V. Bhaskara, and K. Konstantinides, Image and Video Compression Standards Algorithms and Architectures, Boston: Kluwer Academic, 1997.

[8] D. A. Patterson, and J. L. Hennessy, Computer Organization \& Design. The Hardware/Software Interface, San Francisco: Margan Kaufmann, 1994.

[9] W. B. Pennebaker, and J. L. Mitchel, JPEG Still Image Data Compression Standard, New York: Van Nostrand Reinhold, 1993.

[10] M. A. Bayoumi, and E. E. Swartzlander, eds., VLSI Signal Processing Technology, Boston: Kluwer Academic, 1994.

[11] L. Uhr, et al., eds., Evaluation of Multicomputers for Image Processing, New York: Academic Press, 1986.

[12] S. S. Harold, High-Performance Computer Architecture, Menlo Park: AddisonWesley, 1987. 
[13] G. Kang, M. Choe, and M. Song, "A High Performance CMOS A/D Converter for a Digital Camcorder System," IEEE Transaction on Consumer Electronics, vol. 42, pp. 285-288, Aug. 1996.

[14] R. D. Fellman, "Design and Evaluation of an Architecture for a Digital Signal Processor for Instrumentation Applications." IEEE Transactions on Acoustics Speed and Signal Proceeding, vol. 38, pp. 537-546, 1990.

[15] N. Murata, F. Ukigaya, Y. Eto, N. Sakuraba, and T. Delp, "A Totally Digital Camera System Using Digital Triaxial Transmission," SMPTE Journal, pp. 647652, Oct. 1996.

[16] T. Araki, M. Toyokura, M. Wakamori, and K. Aono, "The Architecture of a Vector Digital Signal Processor for Video Coding," IEEE ICASSP vol. 5, pp. 681-684, 1992.

[17] T. Akiyama, H. Aono, K. Aoki, and et al, "MPEG2 Video CODEC Using Image Compression DSP," IEEE Transactions on Consumer Electronics, vol. 40, pp. 466-472, Aug. 1994.

[18] Y. Takemura, K. Suda, H. Serita, and et al, "HDTV Compact Digital Camera Using 2/3" 1.3M CCD Image Sensor," IEEE Transaction on Consumer Electronics, vol. 41, pp. 81-86, Feb. 1995.

[19] T. Leacock, R. Topper, J. Kacke, L. Dischert, L. Waller, and A. Zortea, "An HDTV Digital Camera Processor," SMPTE Journal, pp. 580-585, Sept. 1994.

[20] H. Zen, S. H. Kim, J. H. Jang, H. S. Chun, and C. H. Lee, "A New Digital Camera Processor with Separated Y and C Gamma Control," IEEE Transaction on Consumer Electronics, vol. 40, pp. 610-614, Aug. 1994.

[21] E. A. Lee, "Programmable DSP Architectures: Part I," IEEE ASSP Magazine pp. 4-19, Oct. 1988.

[22] K. Ano, M. Toyokura, T. Araki, A. Ohtani, H. Kodama, and K. Okamoto, "A Video Digital Signal Processor with a Vector-Pipeline Architecture," IEEE Journal of Solid-State Circuits vol. 27, pp. 1886-1893, Dec. 1992.

[23] M. Maruyama, H. Nakahira, T. Araki, S. Sakiyama, Y. Kitao, K. Aono, and H. Yamada, "An Image Signal Multiprocessor on a Single Chip," IEEE Journal of Solid-State Circuits, vol. 25, pp. 1476-1483, Dec. 1990. 
[24] R. H. J. Bloks, "The IEEE-1394 High Speed Serial Bus," Philips Journal of Research vol. 50, pp. 209-216, 1996.

[25] T. Araki, T. Toyokura, T. Akiyama, H. Takeno, B. Wilson, and K. Aono, "Video DSP Architecture for MPEG2 CODEC," IEEE ICASSP, vol 2, pp. 417-420, 1994.

[26] M. Toyokura, M. Saishi, S. Kurohmaru, and et al. "A Video DSP with a Macroblock-Level_Pipeline and a SIMD Type Vector-Pipeline Architecture for MPEG2 CODEC," Proceedings IEEE International Solid-State Circuits Conference, pp.74-75, 1994.

[27] Motorola, MPC82x User Manual, www.motorola.com.

[28] TI, TMS320C8x User Manual, www.ti.com.

[29] Hitachi, SH-DSP SH7410 User Manual, www.hitachi.com.

[30] LSI Logic, DCAM-101 User Manual, www.lsilogic.com.

[31] K. Sayood, Introduction to Data Compression, San Francisco: Morgan Kaufmann, 1996.

[32] G. C. Holst, CCD Arrays, Cameras, and Displays, Winter Park: JCD, 1996.

[33] LSI Logic, LS647XX User Manual, www.lsilogic.com.

[34] NEC CCD Data Book.

[35] Eastman Kodak web site, www.kodak.com.

[36] FlashPix Specification.

[37] USB User Manual, www.intel.com. 


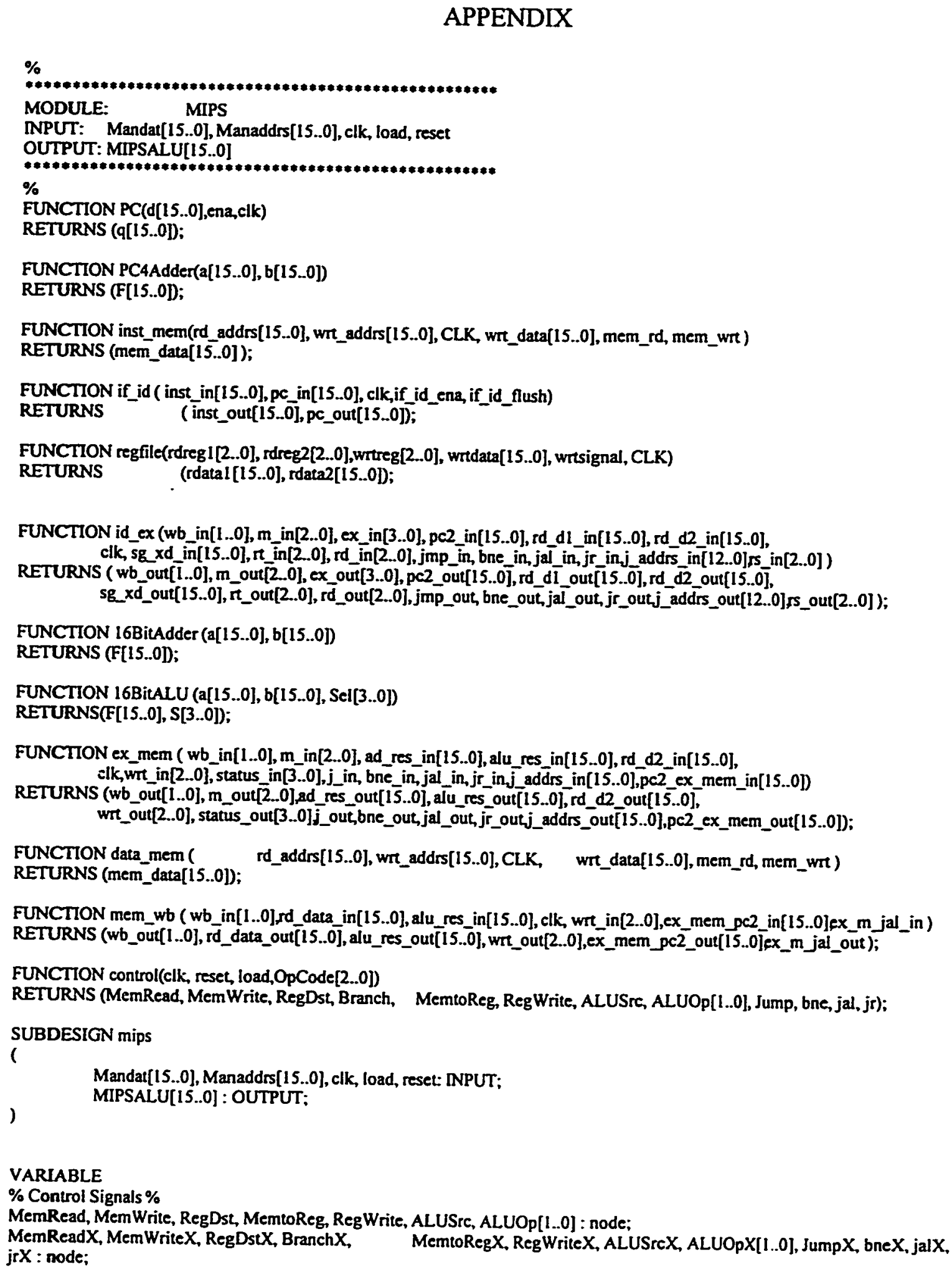




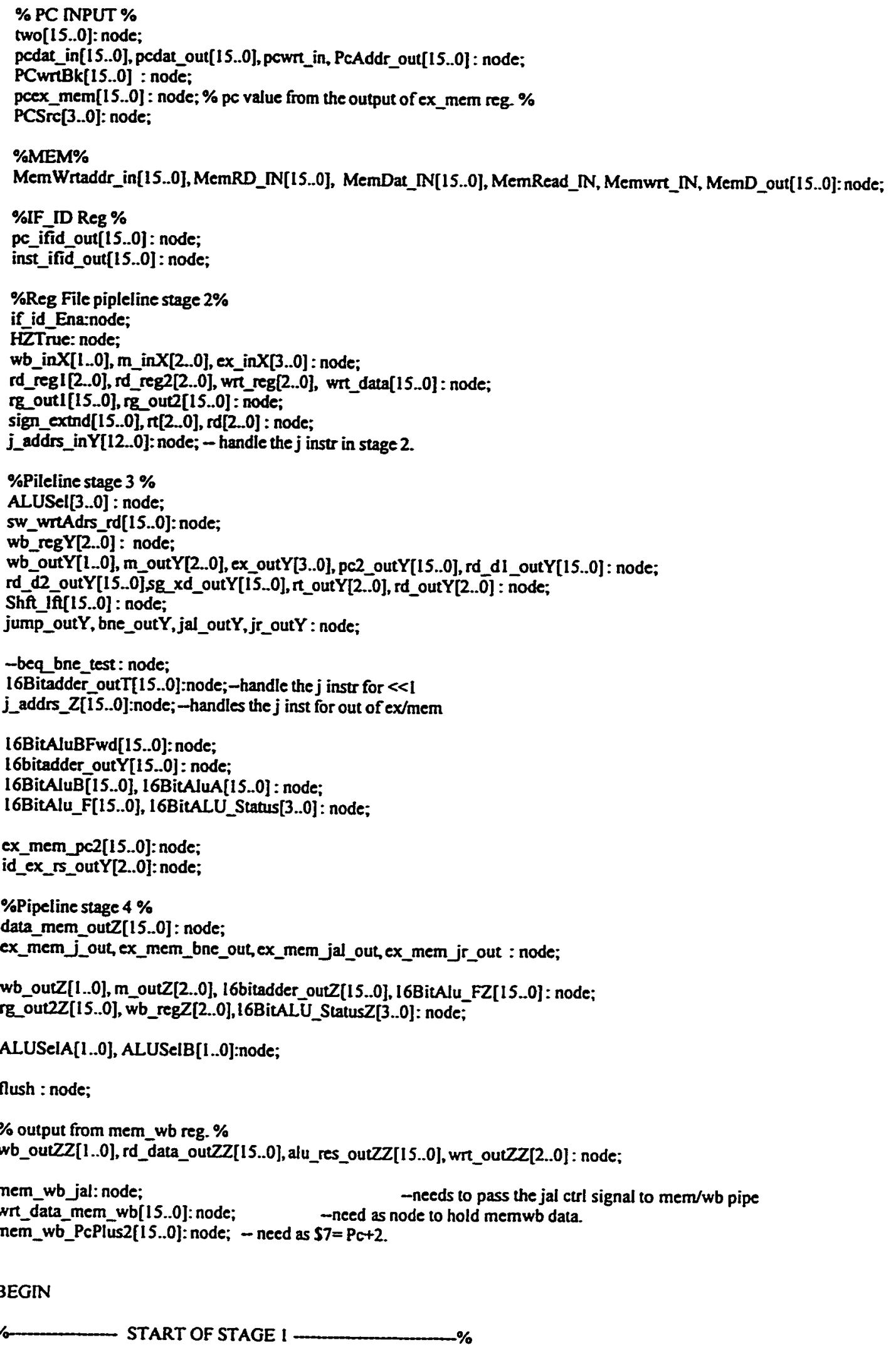




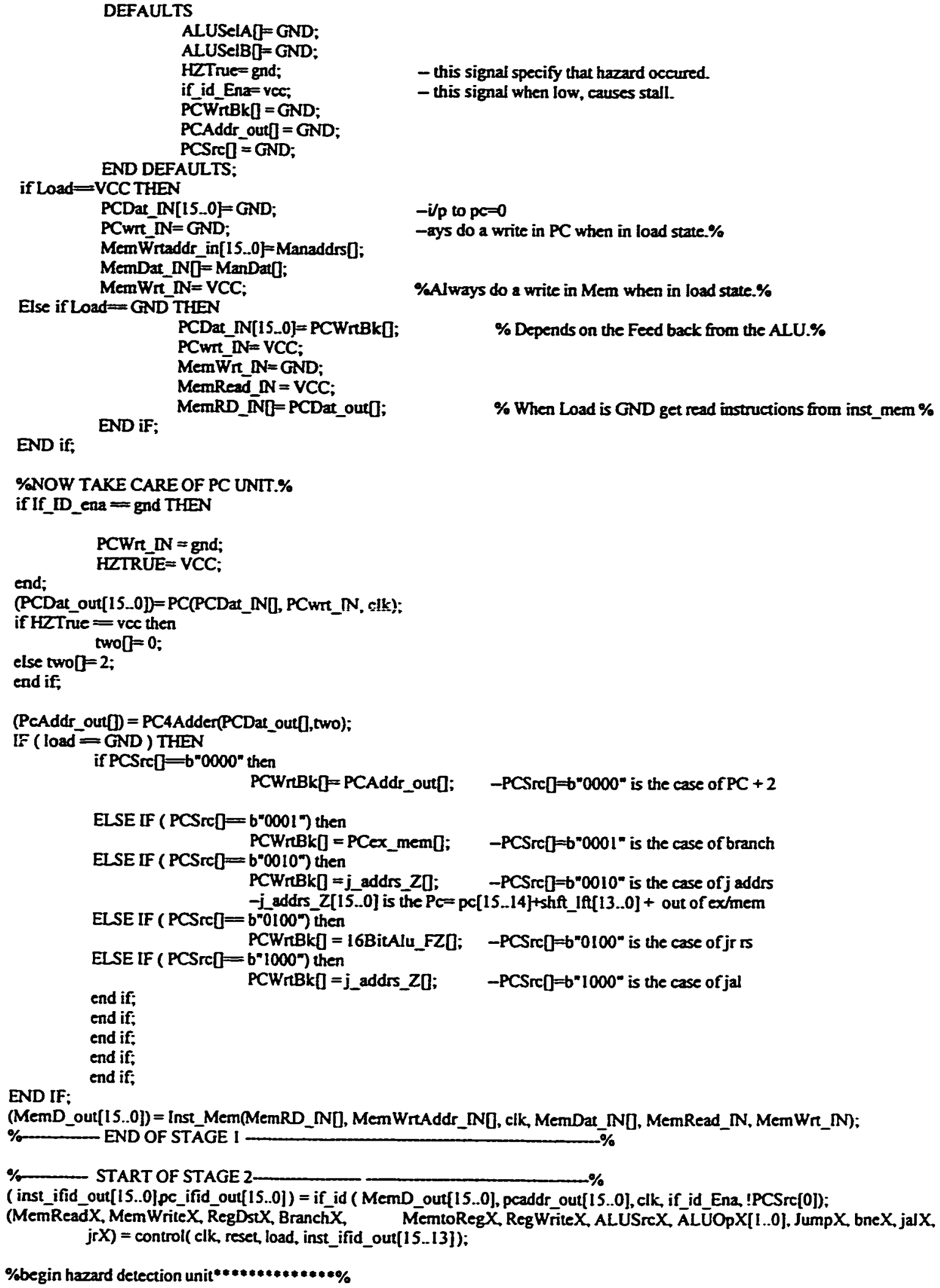

(MemD_out[15..0]) = Inst_Mem(MemRD_IN], MemWrtAddr_IN], clk, MemDat_IND, MemRead_IN, MemWr_IN);

\%begin hazard detection unit"******e**.e.*\%\% 
if ((wb_outY $[1]=V C C)$ and (m_out $Y[1]=v c c)$ ) Then

if ( (\%id/wrtregRT\% rt_outY[2.0]=inst_ifid_out[12..10]\%ifled.rdRegl\%)or

(\%id/wrtregRT\% n_outY[2..0] inst_ifid_out $[9 . .7] \%$ ifled.rdReg2\%)) THEN

if id Ena=gnd:

HZTrue $=$ vec;

(MemReadX, MemWriteX, RegDstX, BranchX, MemtoRegX, RegWriteX.

end if:

ALUSrcX, ALUOpX[1..0], JumpX, bneX, jalX, jrX )= gnd;

else

if id_Ena=vce;

HZ̄ True= gnd;

PCWr_ $\mathrm{NN}=$ vec;

end if;

if (PCSre $\square !=b^{\prime \prime 0000 ") ~ T h e n ~}$

(MemReadX, MemWriteX, RegDstX, BranchX, MemtoRegX, RegWriteX, ALUSreX, ALUOpX[1..0], JumpX, bneX, jalX, jrX $\quad$ ) = gnd; end if;

if( inst_ifid_out[15..13] $=b^{\text {"000") then }}$

iff inst_ifid_out $\left.[3.0]=b^{\prime \prime} 1010^{\prime \prime}\right)$ then

else

$\mathrm{jrX}=\mathrm{vec}$;

end if;

end if;

jrX $=$ gnd;

wb_inX[1] = RegWriteX;

wb_inX[0] = MemToRegX;

$m_{-}$in $X[2]=B$ ranchX;

$m \_$inX[1] $=$MemReadX

$m_{-}$in $X[0]=$ MernWriteX;

ex inX[3] = RegDstx:

ex_inX[2] = ALUOpX[1];

ex inX[1] = ALUOpX[0]:

ex_inX[0]=ALUSreX;

sign_extnd $[6.0]=$ inst_ifid_out $[6.0]$;

sign_extnd [15..7] = gnd;

$\mathrm{r}[\mathrm{I}=$ inst_ifid_out [9..7];

rd] = inst_ifid_out [6..4];

$\%$ Inputs to Reg file \%

rd_regl[2..0] = inst_ifid_out[12..10];

rd_reg2 $[2.0]=$ inst_ifid_out $[9 . .7]$;

(rg_out1[], rg_out20)=Regfile(rd_reg10,rd_reg2], wr_reg[, wr_data[], RegWrite, clk);

(wb_outY[1..0], m_outY[2..0], ex_outY[3..0],pc2_outY[15.0], rd_dl_outY[15..0],rd_d2_outY[15..0]sg_xd_out $Y[15 . .0]$,

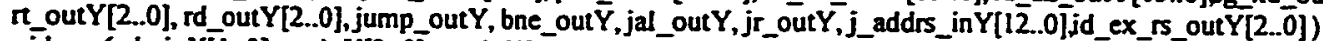

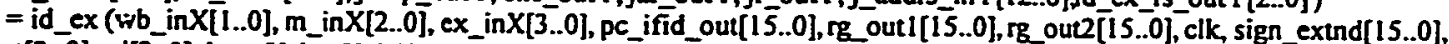

$\mathrm{rt}[2 . .0], \mathrm{rd}[2 . .0]$, jumpX, bneX, jalX, jrX, inst_ifid_out[12.0]inst_ifid_out[12..10]):

END OF STAGE 2

$\%$ START OF STAGE 3

$\%$

- This takes care of sw forwarding

- if $\pi_{-}$ex_mem $=\pi$ id_ex and is $S W$ instr then need forward the result of ex_mem from the alu.

-rtex_mem=wb_regZ[]

$-r_{-}$id_ex $=r_{\text {_out }} Y 0$

$-m_{-}$out $Y[0]=\bar{S} W$ instr signal.

if ( $\left(w b \_r e g Z D=r_{-}\right.$out $\left.Y D\right)$ and $\left(m_{-}\right.$out $\left.Y[0]=v c c\right)$ ) then sw_wrtAdrs_rd] = 16BitAlu_FZ[15..0]; 


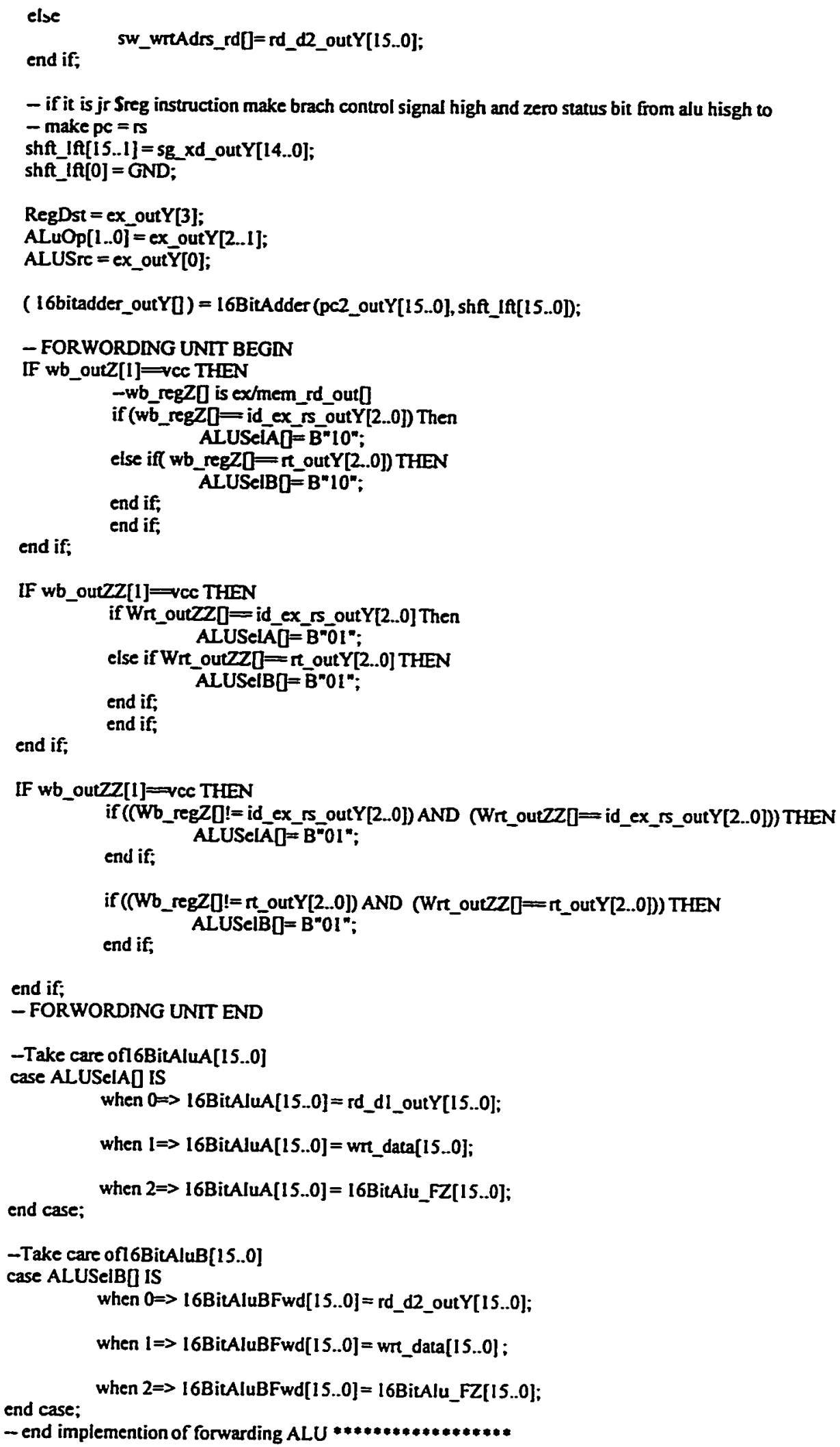




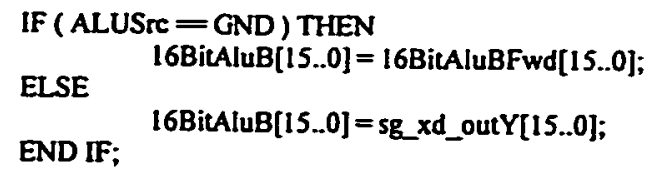

CASE AluOp[1...0] IS

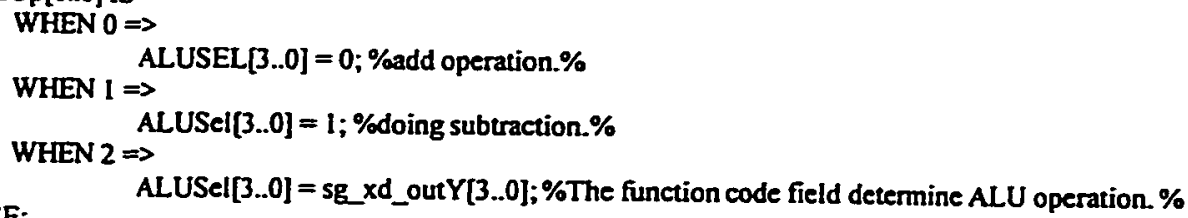

(wb_outZ[1..0], m_outZ[2..0], 16bitadder_outZ[, 16BitAlu_FZ[15..0], rg_out2Z[15..0],wb_regZ[2..0],16BitALU_StatusZ[3..0], ex_mem_j_out, ex_mem_bne_out, ex_mem_jal_out, ex_mem_jr_out,j_addrs_žzis..0], ex_mem_pc2[15..0]

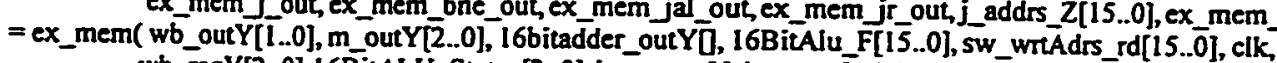
wb_regY[2..0],16BitALU_Status[3..0],jump_outY, bne_outY,jal_outY,jr_outY, $\overline{16}$ BitAdder_outT[15..0],
pc2_outy[15..0]);

if ex_mem jr_out $=$ vec then 


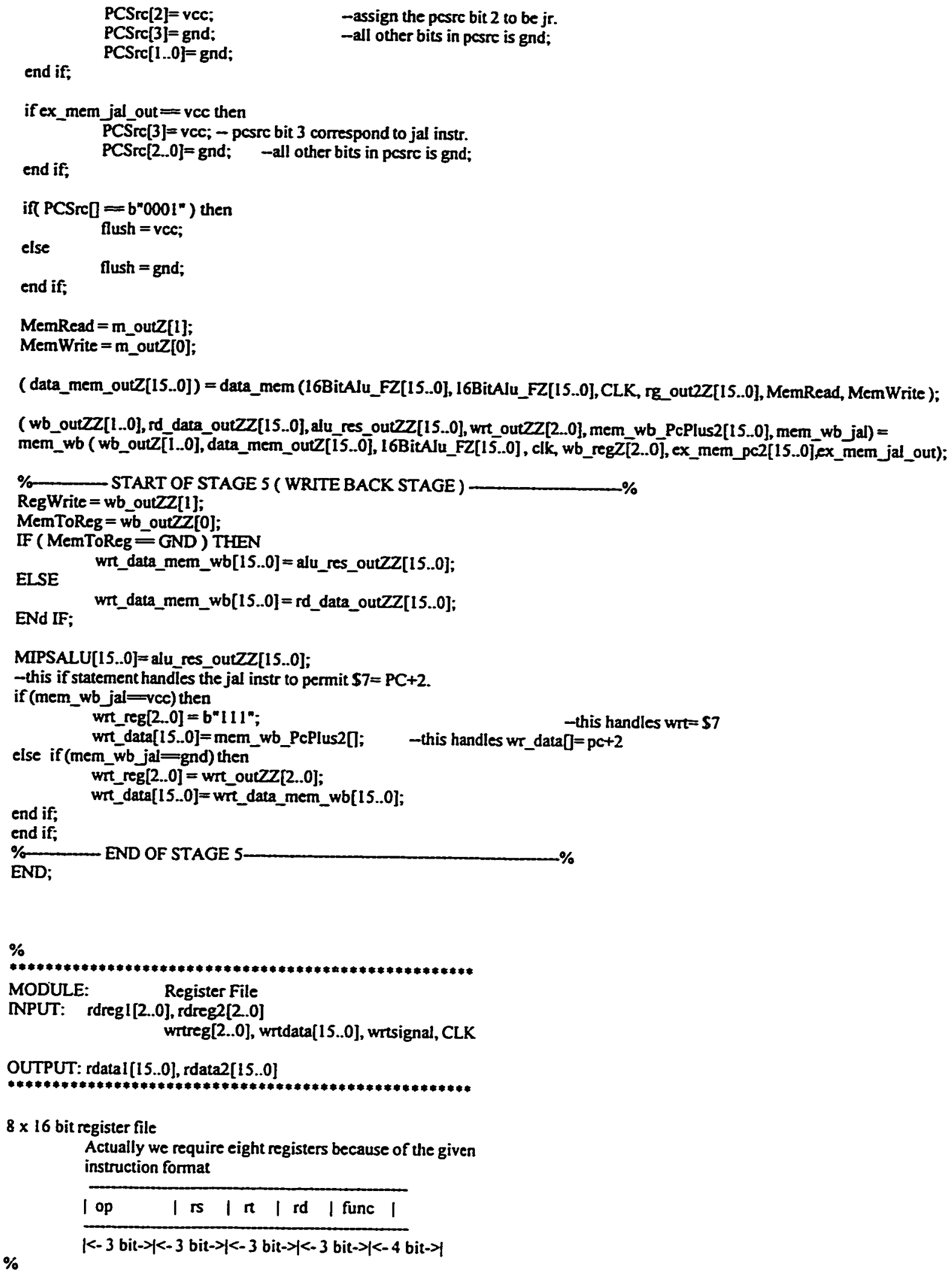




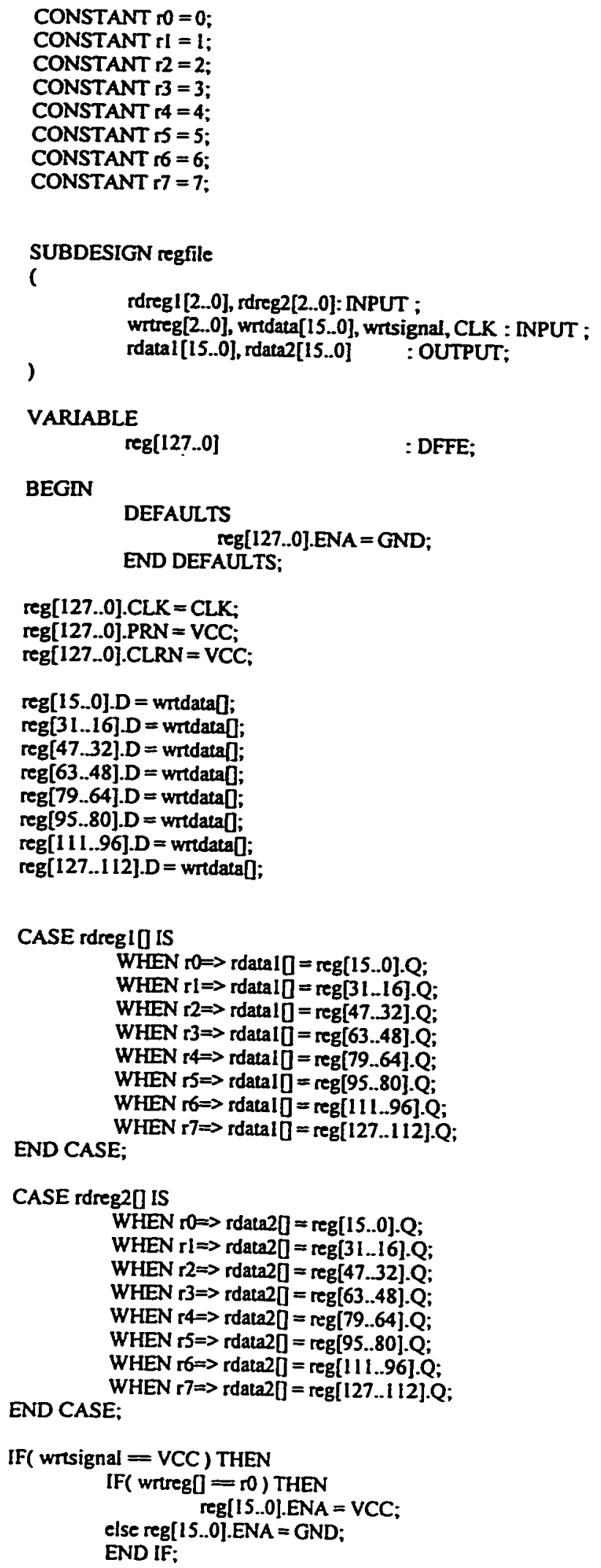




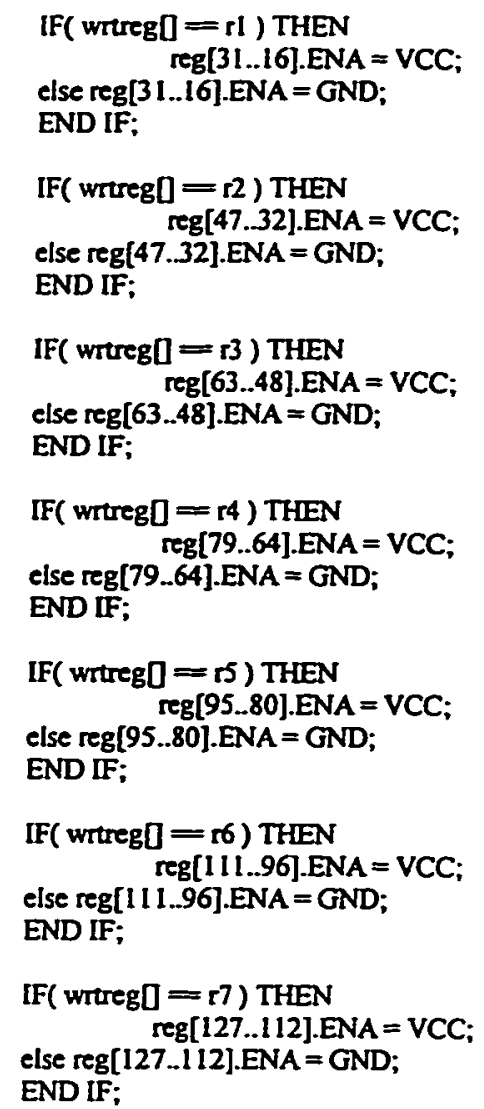

END IF;

END;

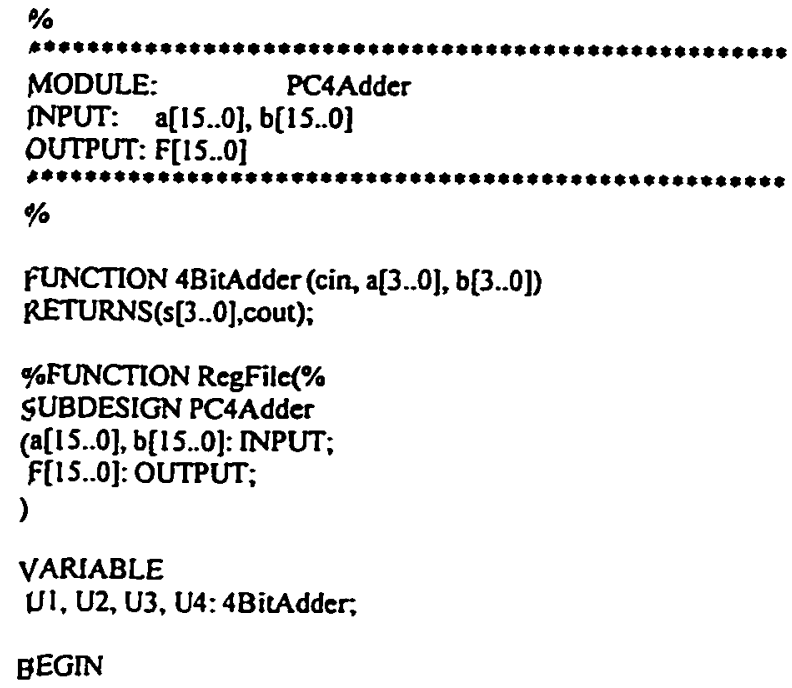


U1.b[3..0 $=b[3 . .0]$

\%assign Ul's input a[3..0] $=a[7 . .4]$, the input from the register file\% U2.cin = U1 .cout; \%casecading UI and U2.\%

U2.a[3..0] $=a[7 . .4]$;

$\%$ assign Ul's input $a[3 . .0]=b[7 . .4]$, the input from the register file\% U2.b[3.0] $=b[7 . .4]$

$\%$ assign UI's input a[3..0] $=a[11 . .8]$, the input from the register file\% U3. cin = U2. cout; \%U3's cin = the cout of $\mathrm{W2} . \%$

U3.a[3..0]=a[11..8];

$\%$ assign Ul's input $a[3 . .0]=b[11 . .8]$, the input from the register file\% U3.b[3..0 $=b[11 . .8]$;

\%assign UI's input $a[3 . .0]=a[15 . .12]$, the input from the register file\% U4.cin = U3.cout; \%casecading U3and U4.\%

U4.a[3.0] $=\mathrm{a}[15 . .12]$

\%assign UI's input $a[3 . .0]=b[15 . .12]$, the input from the register file\% U4.b[3..0 $]=b[15 . .12]$;

$\%$ Now produce the Function bits and tie status.\%

$F[3 . .0]=U 1 . S[3 . .0]$;

$F[7 . .4]=U 2 . S[3 . .0]$

$F[11 . .8]=U 3 . S[3 . .0]$;

END;

$F[15 . .12]=U 4 . S[3.0]$;

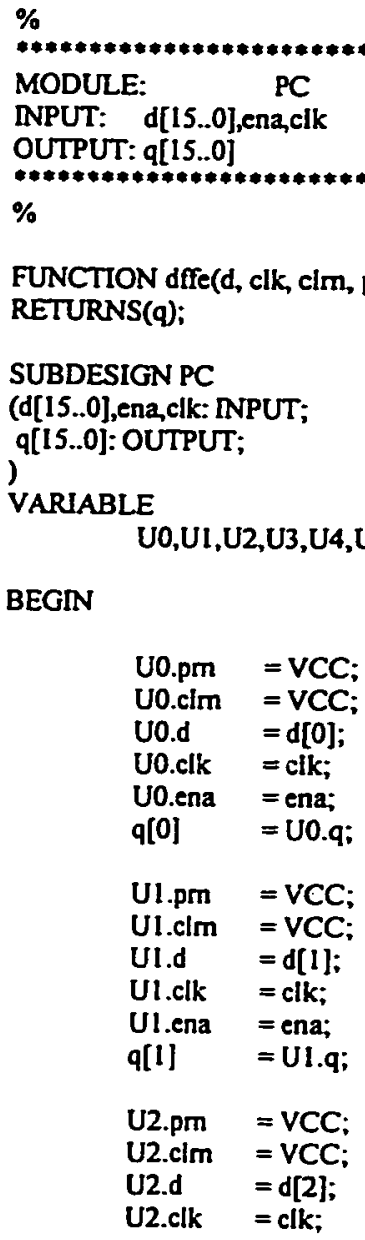

BEGIN

$$
\begin{array}{ll}
\text { U0.pm } & =v C C ; \\
\text { U0.clm } & =V C C ; \\
\text { U0.d } & =d[0] ; \\
\text { U0.clk } & =\text { clk; } \\
\text { U0.ena } & =\text { ena; } \\
\text { q[0] } & =U 0 . q ; \\
\text { U1.pm } & =V C C ; \\
\text { U1.clm } & =v C C ; \\
\text { U1.d } & =d[1] ; \\
\text { U1.clk } & =\text { clk; } \\
\text { U1.ena } & =\text { ena; } \\
\text { q[1] } & =U 1 . q ; \\
\text { U2.pm } & =v C C ; \\
\text { U2.cim } & =V C C ; \\
\text { U2.d } & =d[2] ; \\
\text { U2.clk } & =\text { clk; }
\end{array}
$$




$$
\begin{aligned}
& \text { U2.ena = ena: } \\
& \text { q[2] = U2.q; } \\
& \text { U3.pm = VCC; } \\
& \text { U3.clm = VCC; } \\
& \text { U3.d }=\mathrm{d}[3] \text {; } \\
& \text { U3.clk = clk; } \\
& \text { U3.ena = ena; } \\
& \text { q[3] = U3.q; } \\
& \text { U4.pm = VCC; } \\
& \text { U4.cim = VCC; } \\
& \text { U4.d = d }[4] \text {; } \\
& \text { U4.clk = clk; } \\
& \text { U4.ena = ena; } \\
& \mathrm{q}[4]=U 4 . \mathrm{q} \text {; } \\
& \text { U5.pm = VCC; } \\
& \text { US.clm = VCC; } \\
& \text { U5.d }=\mathrm{d}[5] \text {; } \\
& \text { US.clk =clk; } \\
& \text { U5.ena = cna; } \\
& q[5] \text { = US.q; } \\
& \begin{array}{ll}
\text { U6.pm = VCC; } & \\
\text { U6.clm } & =v C C ; \\
\text { U6.d } & =d[6] \\
\text { U6.clk } & =\text { clk; } \\
\text { U6.ena } & =\text { ena; } \\
\text { q[6] } & =\text { U6.q; }
\end{array}
\end{aligned}
$$




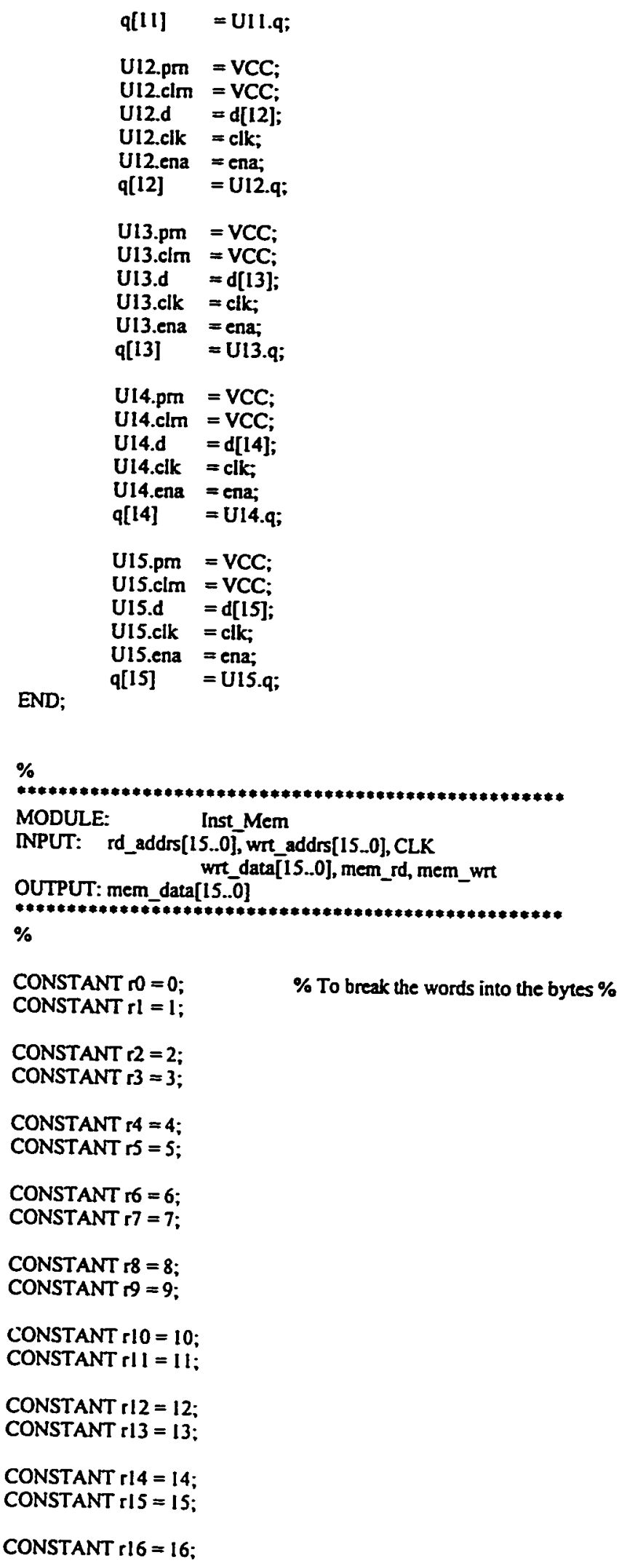




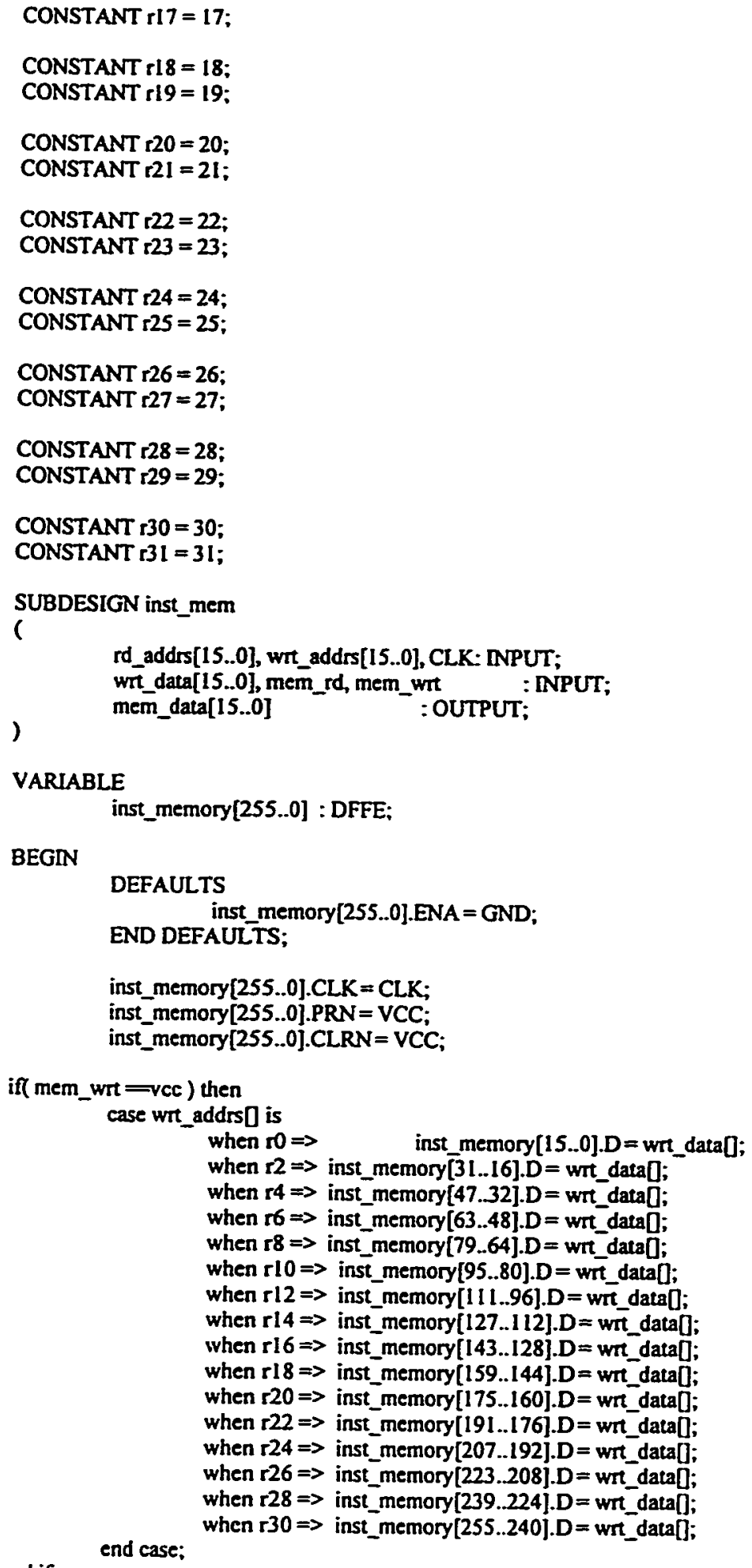


END IF; END CASE:

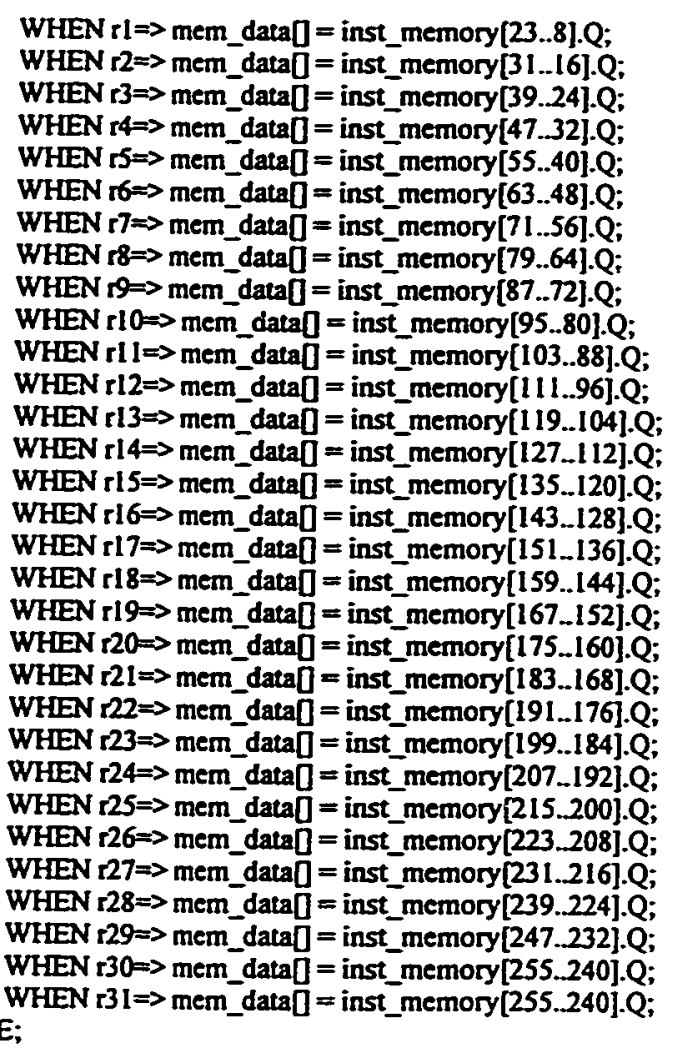

IF ( mem_wrt $=$ VCC $)$ THEN

IF ( wr_addrs $[=r 0)$ THEN

inst_memory[15.0].D = wrt_dataQ;

inst_memory[15..0].ENA = VCC;

else inst_memory[15..0].ENA = GND;

END IF;

IF(wrt_addrs $[=r l$ ) THEN

inst_memory [23..8].D = wit_data[];

inst_memory[23..8].ENA = VCC;

else inst_memory[23..8].ENA = GND;

END IF;

IF( wrt_addrs $[=r 2$ ) THEN

inst_memory[31..16].D = wrt_data[];

inst_memory[31..16].ENA = VCC;

else inst_memory[31..16].ENA = GND;

END IF;

IF( wrtaddrs $]=r 3$ ) THEN

inst_memory[39.24].D = wrt_data[; inst_memory[39.24].ENA = V $C C$;

else inst_memory[39..24].ENA = GND;

END IF;

IF (wr_addrs $]=r 4$ ) THEN

inst_memory $[47.32] . D=w \pi$ data[;

inst_memory[47.32].ENA = VCC:

else inst_memory[47..32].ENA = GND;

END IF:

IF( wrt_addrs $]=$ r5) THEN 


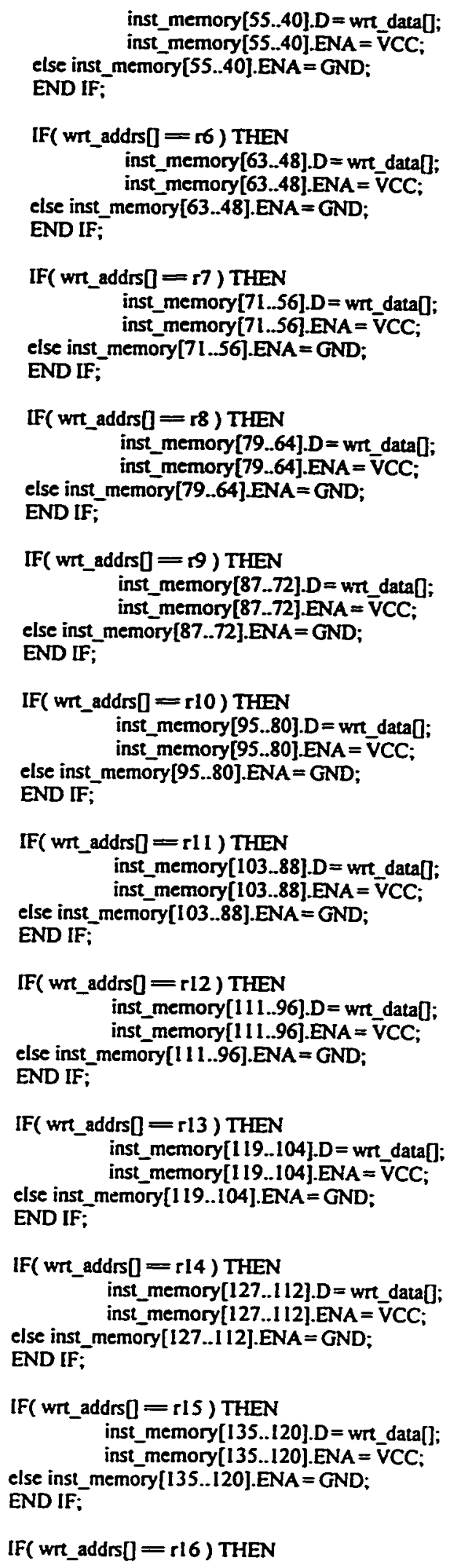

IF(wrt_addrs []$=$ rl6) THEN 


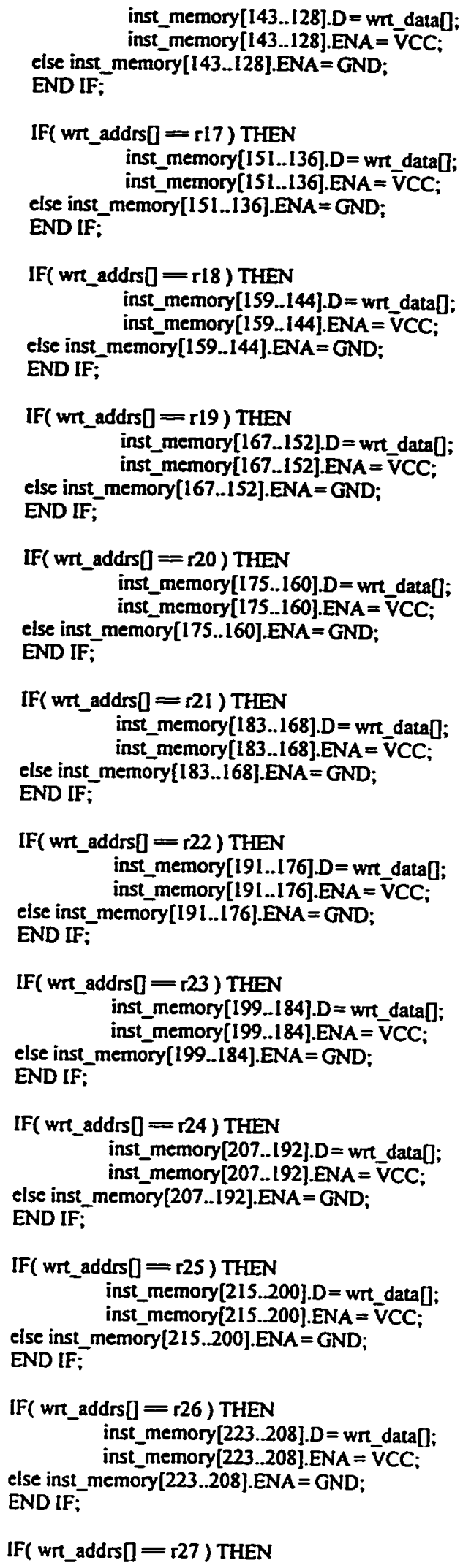

IF( wn_addrs $]=$ r27) THEN 


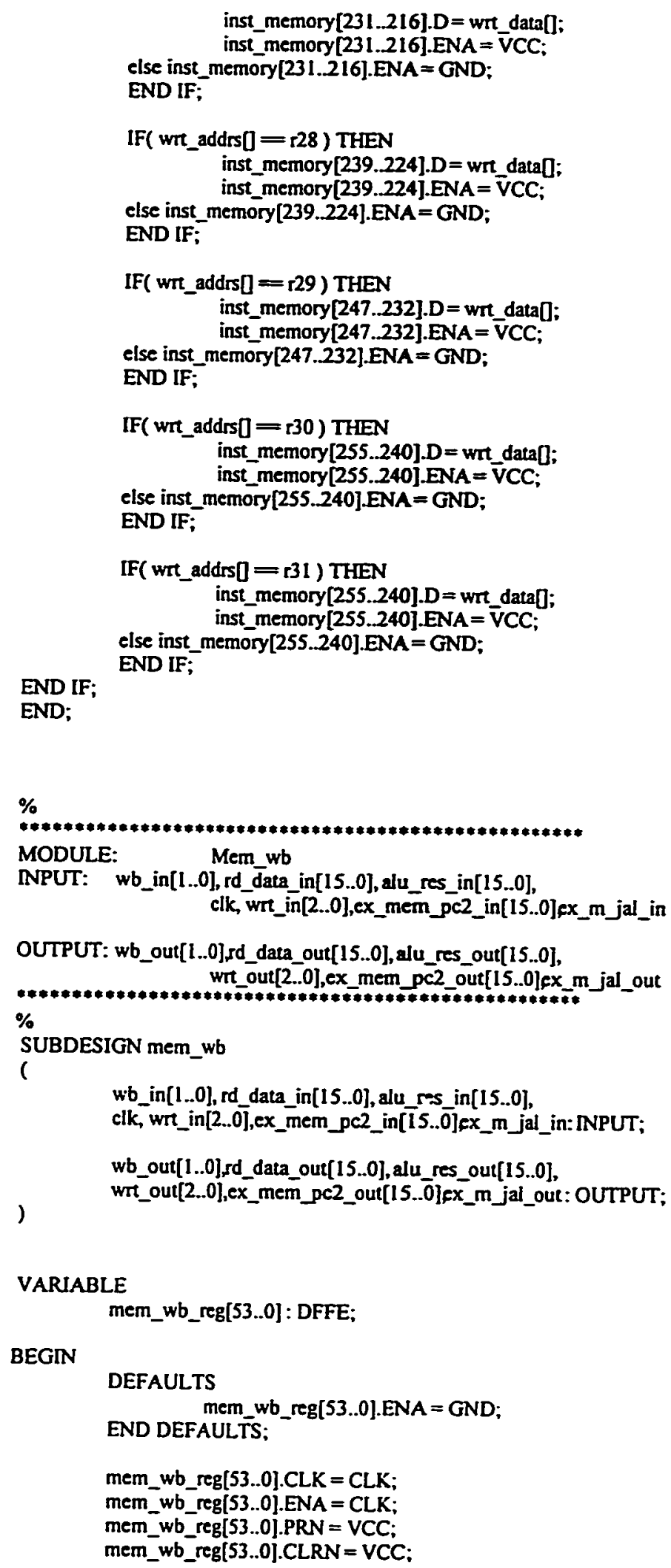

\section{VARIABLE}

mem_wb_reg[53..0] : DFFE;

BEGIN

DEFAULTS

men_wb_reg[53..0].ENA = GND;

END DEFAULTS;

mem_wb_reg[53..0].CLK $=$ CLK;

mem_wb reg[53..0].ENA $=$ CLK;

mem_wb_reg[53..0].PRN $=$ VCC:

mem_wb_reg[53..0].CLRN $=$ VCC; 
sg_xd_out[15..0], rt_out[2.0], rd_out[2.0]

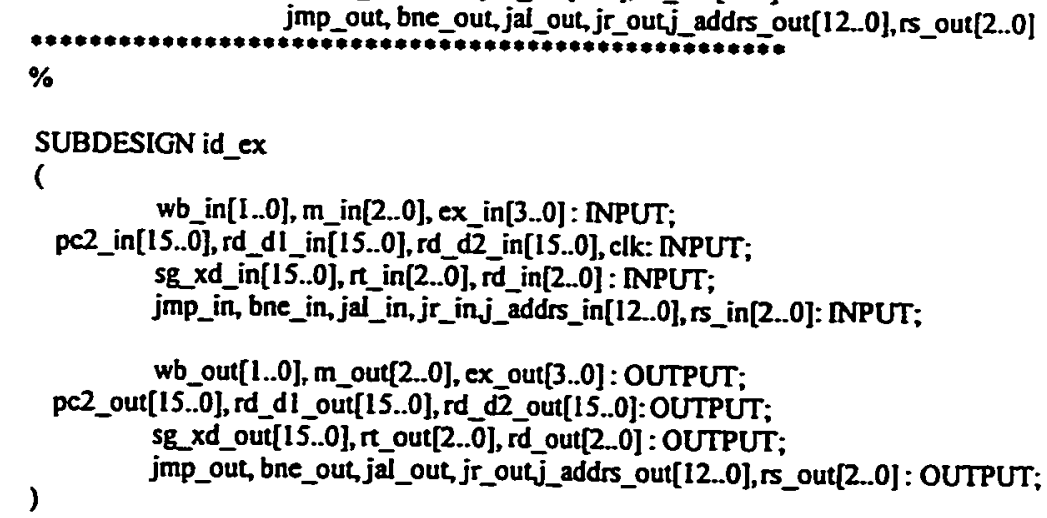

END: 


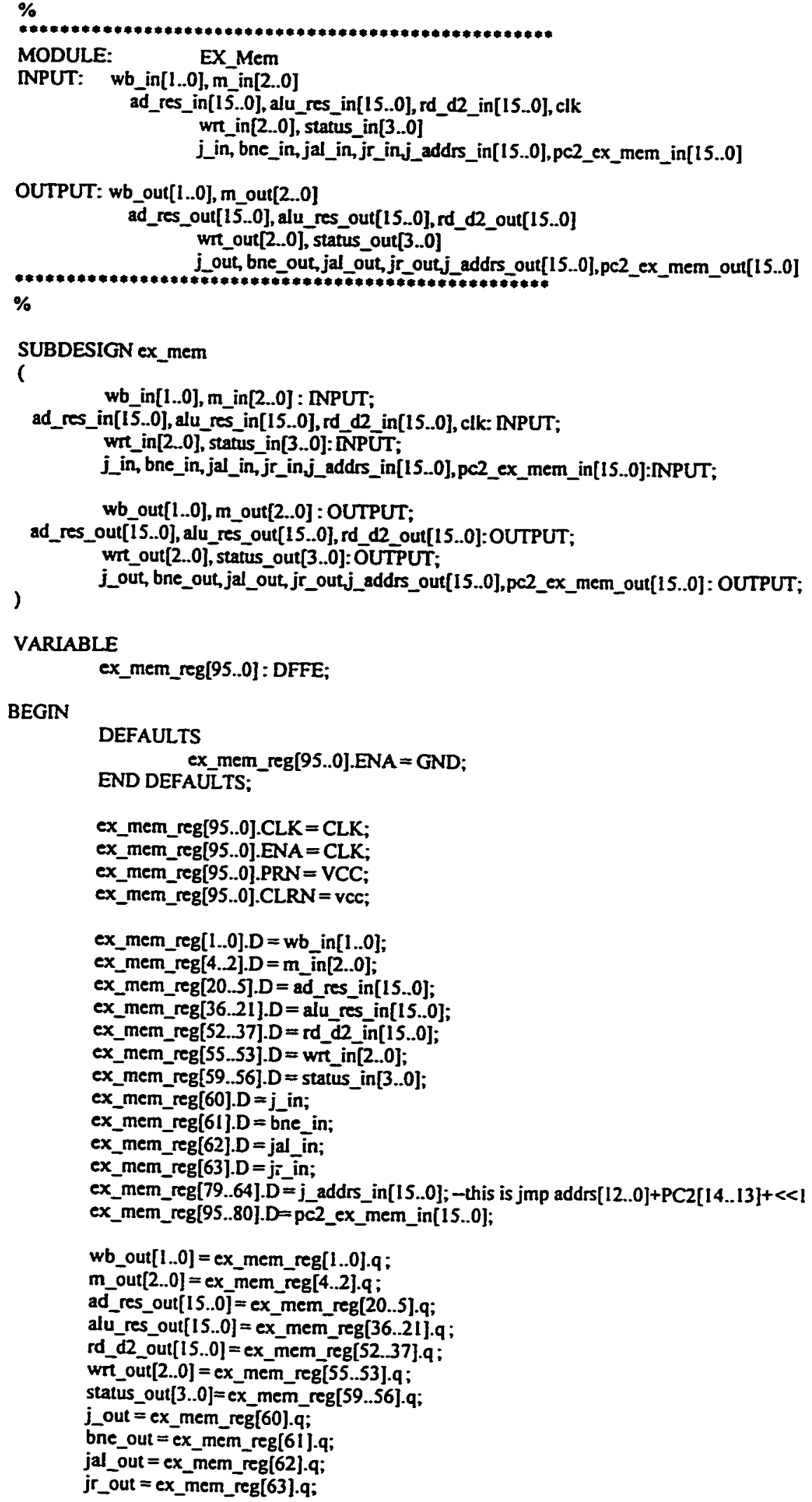


END;

j_addrs_out $\left[=e x \_m e m \_r e g[79.64] . q ;\right.$-this is jmp addrs[12..0]+PC2[14..13]+ $2<1$

pC2_ex_mem_out] $=$ ex_mem_reg[95..80].q;

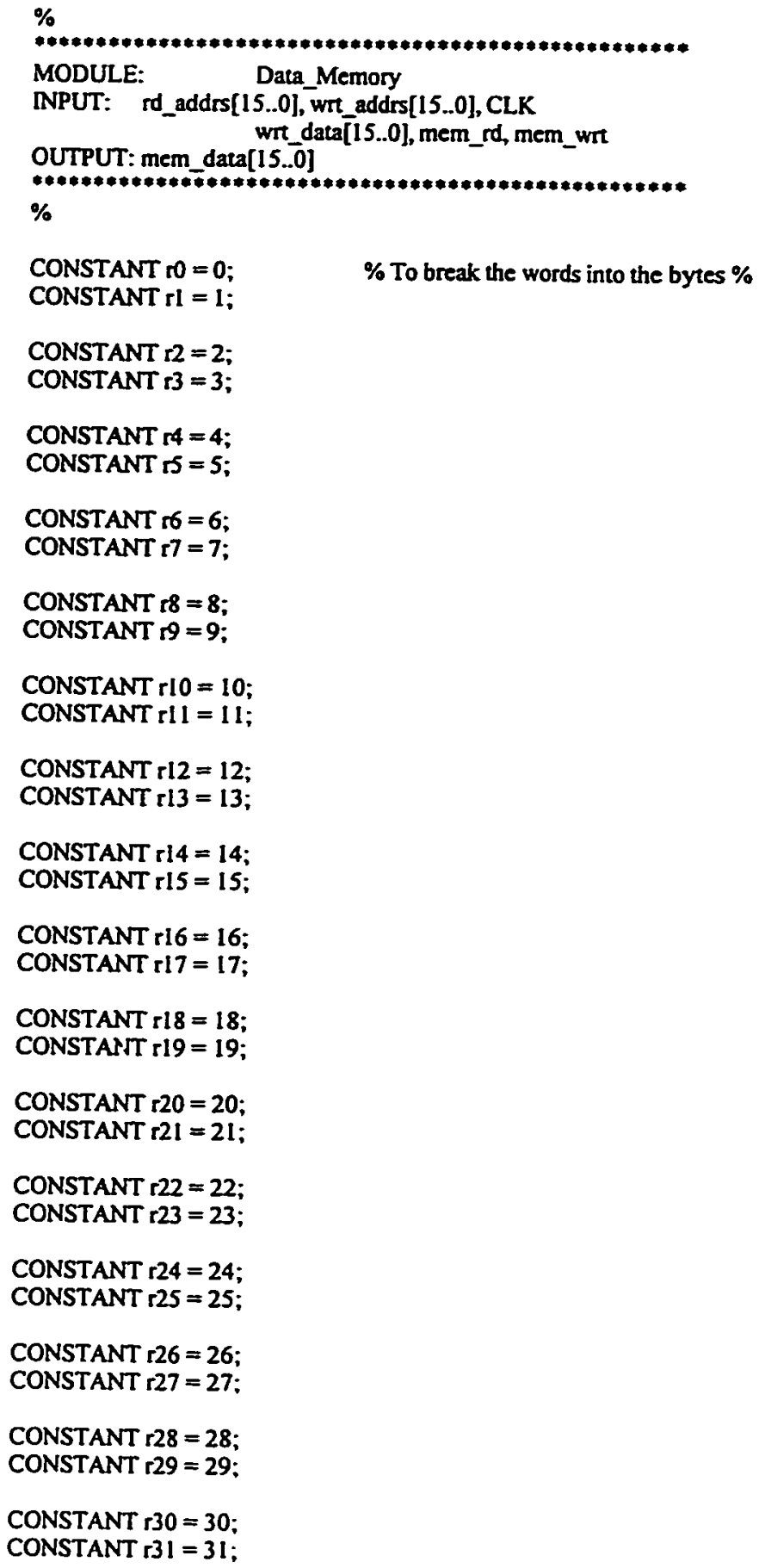


rd_addrs[15..0], wrt_addrs[15..0], CLK: INPUT;

wn_data[15..0], mem_rd, mem_wrt : INPUT;

)

$$
\text { mem_data[15..0] }
$$

VARIABLE

data_memory[255..0] : DFFE;

BEGIN

DEFAULTS

data_memory[255..0].ENA = GND;

END DEFAULTS;

data_memory[255..0].CLK $=\mathrm{CLK}$;

dala memory[255..0].PRN $=$ VCC;

data_memory[255..0].CLRN = VCC;

if( mem_wrt $=v c c)$ then

case wrt_addrs $]$ is

$$
\begin{aligned}
& \text { when } \mathrm{r} 0 \Rightarrow \text { data_memory [15..0].D = wrt_data }[ \\
& \text { when } r 2 \Rightarrow \text { data memory }[31 . .16] . D=\text { wrt data }] \\
& \text { when } r 4 \Rightarrow \text { data_mernory[47.32].D = wrtdata }[ \\
& \text { when } r 6 \Rightarrow \text { data_memory [63.48].D = wrtdata }[\text {; } \\
& \text { when } \mathrm{r} 8 \Rightarrow \text { data_memory[79..64].D = wrt_data } \overline{0} \text {; } \\
& \text { when } \mathrm{r} 10 \Rightarrow \text { data_memory[95..80].D = wrt_data[; } \\
& \text { when } r 12 \Rightarrow \text { data_memory[111..96].D }=\text { wrז_data]; } \\
& \text { when } \mathrm{rl} 4 \Rightarrow \text { data_memory[127..112].D = wrt_data[ } \\
& \text { when } \mathrm{rl6} \Rightarrow \text { data_memory[143..128].D = wrt_data[; } \\
& \text { when } r 18 \Rightarrow \text { data_memory[159..144].D }=\text { wrt_data } \\
& \text { when } r 20 \Rightarrow \text { data_memory [175..160].D = wrt_data } \\
& \text { when } r 22 \Rightarrow \text { data_memory[191...176]. } D=w r \text { data } \\
& \text { when r24 } \Rightarrow \text { data_memory[207...192].D = wrt_data[; } \\
& \text { when } r 26 \Rightarrow \text { data_memory[223.208].D }=\text { wr_data } \\
& \text { when } \mathrm{r} 28 \Rightarrow \text { data_memory[239.224].D }=\text { wrt data]; }
\end{aligned}
$$

end case

end if;

IF (mem_rd $=$ VCC $)$ THEN

CASE rd_addrs] IS

WHEN $\mathrm{r} 0=>$ mem_data $]=$ data_memory $[15 . .0] . Q$;

WHEN $\mathrm{rl} \Rightarrow$ mem_data $]=$ data_memory[23..8].Q;

WHEN r2 $\Rightarrow$ mem_data] = data_memory[31..16].Q;

WHEN $r 3=>$ mem_data $]=$ data_memory[39..24].Q;

WHEN $r 4 \Rightarrow$ mem_data[ $=$ data_memory[47.32].Q;

WHEN $r 5 \Rightarrow$ mem_data[ $=$ data_memory [55..40].Q;

WHEN $16 \Rightarrow$ mem_data $D=$ data_memory[63..48].Q;

WHEN r7 $\Rightarrow$ mem_data] = data_memory[71..56].Q;

WHEN $r 8 \Rightarrow$ mem_data[] = data_memory[79..64].Q;

WHEN $r \Rightarrow$ mem_data $=$ data_memory[87..72].Q;

WHEN rl $0 \Rightarrow$ mem_data $]$ = data_memory[95..80].Q;

WHEN rl l $\Rightarrow$ mem_data $[$ = data_memory[103..88].Q;

WHEN $\mathrm{rl} 2 \Rightarrow$ mem_data $]=$ data_memory $[111 . .96] . Q$.

WHEN $\mathrm{rl} 3 \Rightarrow$ mem_data $]=$ data_memory [119..104].Q;

WHEN $\mathrm{rl} 4 \Rightarrow$ mem_data[] $=$ data_memory [127.. I12].Q;

WHEN r $15 \Rightarrow$ mem_dataD = data_memory [135..120].Q;

WHEN $\mathrm{rl} 6 \Rightarrow$ mem_data[] = data memory[ [43..128].Q;

WHEN rl 7 $=>$ mem_data $]=$ data_memory[151..136].Q;

WHEN r1 $8 \Rightarrow$ mem_data $[$ = data_memory[159..144].Q;

WHEN r19=> mem_data[ = data_memory[167..152].Q;

WHEN $r 20 \Rightarrow$ mem_data $[=$ data_memory $[175 . .160] . Q$;

WHEN r $21 \Rightarrow$ mem_data []$=$ data_memory[183..168].Q;

WHEN $r 22 \Rightarrow$ mem_data $]=$ data_memory $[191 . .176] . Q$;

WHEN $r 23 \Rightarrow$ mem_data $]$ = data_memory [199..184].Q;

WHEN r24 $\Rightarrow$ mem_data[] = data_memory[207.. 192].Q;

WHEN r25 $=>$ mem_data $[$ = data_memory $[215 . .200] . Q$; 
END IF:

WHEN $r 26 \Rightarrow$ mem_data] $=$ data_memory [223.208].Q;

WHEN r27 $\Rightarrow$ mem_data[ = data_memory[231.216].Q;

WHEN $r 28 \Rightarrow$ mem_data[ $=$ data_memory[239.224].Q;

WHEN r29 $\Rightarrow$ mem_data] = data_memory[247.232].Q;

WHEN r 30 $\Rightarrow$ mem_data[] $=$ data_memory [255.240].Q;

END CASE:

WHEN r3 I $\Rightarrow$ mem_data $[$ = data_memory [255.240].Q;

\section{IF( mem_wrt $=$ VCC $)$ THEN}

IF( wrt_addrs] $=$ r0) THEN

data_memory[15..0].D = wr_data $]$; data_memory[15..0].ENA = V VCC;

else data memory[15..0].ENA = GND;

END IF:

IF (wrt_addrs[ $=$ rl ) THEN

data_memory [23..8].D = wrt data[; data_memory[23..8].ENA = VCC:

else data_memory[23..8].ENA = GND;

END IF;

IF ( wrt_addrs] $=r 2$ ) THEN

data_memory[31..16].D = wr_data [

data_memory[31..16].ENA = VCC;

else data_memory [31..16].ENA = GND;

END IF;

IF ( wrt_addrs $\square==r 3$ ) THEN

data_memory[39.24].D = wrt_data[];

data_memory[39.24].ENA = VCC;

else data_memory[39.24].ENA = GND;

END IF;

IF( wrt_addrs $]=r 4$ ) THEN

data_memory [47..32]. $\mathrm{D}=\mathrm{wr} \_$data $[$; data_memory[47..32].ENA = VCC;

else data_memory[47.32].ENA = GND;

END IF;

IF( wrt_addrs $]=15$ ) THEN

data_memory $[55 . .40] . D=$ wrt_data ; data_memory[55..40].ENA = VCC;

else data_memory[55..40].ENA = GND;

END IF;

IF( wn_addrs] $=$ r6) THEN

data_memory[63..48].D = wrt data[;

data_memory[63..48].ENA = VCC;

else data_memory [63..48].ENA = GND;

END IF;

IF( wr_addrs[] = r7) THEN

data_memory[71..56].D = wrt data $[$;

data_memory[71..56].ENA = VCC

else data_memory[71..56].ENA = GND;

END IF:

IF( wrt_addrs[] $=r 8)$ THEN

data_memory[79..64].D = wrt_data[]

data_memory[79..64].ENA $=\overline{\mathrm{v} C C}$

else data_memory[79..64].ENA = GND; 
END IF;

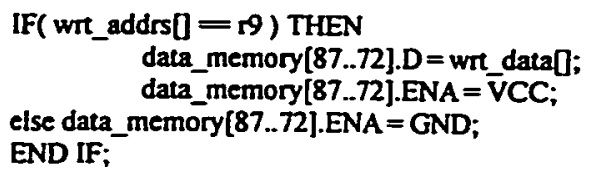




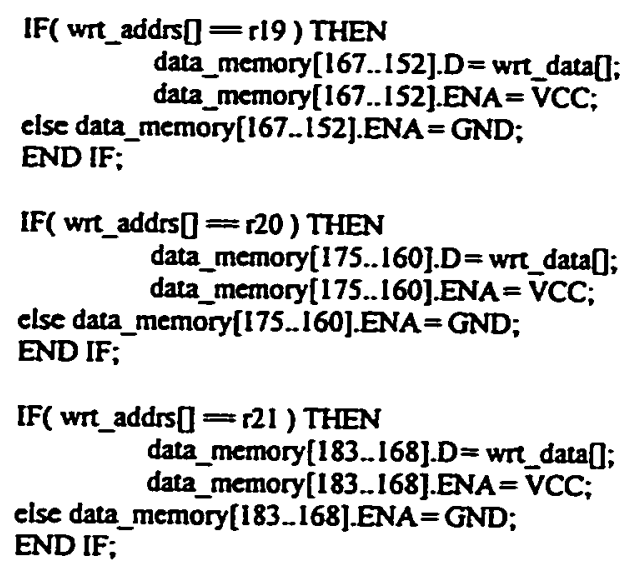

IF ( wrt_addrs $]=r 22$ ) THEN data_memory[191...176].D = wrt_data [; data_memory[191...176].ENA = VCC; else data_memory[191..176].ENA = GND; END IF;

IF (wr_addrs] $=$ r23) THEN data_memory[199..184].D = wrt data]; data_memory[199..184].ENA = vCC; else data_memory[199..184].ENA = GND; END IF;

IF( wrt_addrs $[=$ r24) THEN data_memory[207...192].D = wrt_data[; data_memory[207..192].ENA = VCC; else data_memory[207...192].ENA = GND; END IF;

IF (wrtaddrs $\square=r 25$ ) THEN data_memory [215.200].D = wrt_data]; data_memory[215.200].ENA = VCC; else data_memory[215.200].ENA = GND; END IF;

IF ( wrt_addrs $\mathrm{D}=\mathrm{r26}$ ) THEN data_memory[223.208].D = wrt data[]; data_memory[223.208].ENA = VCC; else data_memory $[223.208] . E N A=$ GND; END IF;

IF (wrt_addrs $]=r 27)$ THEN data_memory[231.216].D = wrt_data[]; data_memory[231.216].ENA = VCC; else data_memory[231..216].ENA = GND; END IF;

IF ( wrt_addrs $]=\mathrm{r} 28$ ) THEN

data_memory[239.224].D = wrt data[; data_memory[239.224].ENA = VCC; else data_memory[239.224].ENA = GND; END IF;

[F( wr_addrs[] $=$ t29) THEN data_memory[247.232].D = wrt_data []; dala_memory [247.232].ENA = VCC: else data_memory[247.232].ENA = GND; 
END IF:

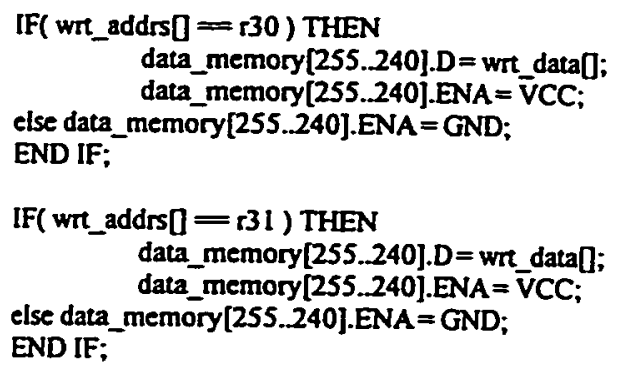

END IF;

END;

$\%$

$* * * * * * * * * * * * * * * * * * * * * * * * * * * * * * * * * * * * * * * * * * * * * * * * * *$

MODULE: $\quad$ Control (State Machine)

INPUT: clk, reset, load, OpCode[2..0]

OUTPUT: MemRead, MemWrite, RegDst, Branch, MemtoReg, RegWrite, ALUSrc, ALUOp[1..0], Jump, bne, jal, jr

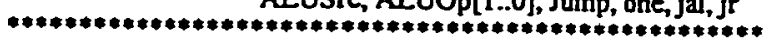

$\%$

CONSTANT $\mathrm{r}=0$;

CONSTANT $\mathrm{rl}=1$;

CONSTANT $\mathrm{r2}=2$;

CONSTANT $\mathrm{r} 3=3$;

CONSTANT $14=4$;

CONSTANT $\mathrm{r}=5$;

CONSTANT $16=6$;

CONSTANT $r 7=7$;

CONSTANT $18=8$;

SUBDESIGN control

(

clk, reset, load, OpCode[2..0]: INPUT;

MemRead, MemWrite, RegDst, Branch, MemtoReg, RegWrite, )

ALUSre, ALUOp[1..0], Jump, bne, jal, jr: OUTPUT

\section{VARIABLE}

ss : MACHINE WITH STATES(ReSt, LdSL, s0 );

BEGIN

CASE ss IS

when Rest $=>$

if (reset $=\mathrm{VCC})$ then

else ss=LdSt;

when LdSt $=>$

end if;

If (load $=\mathrm{VCC}$ ) then

else ss= LdSt;

ss $=50$;

end if; 

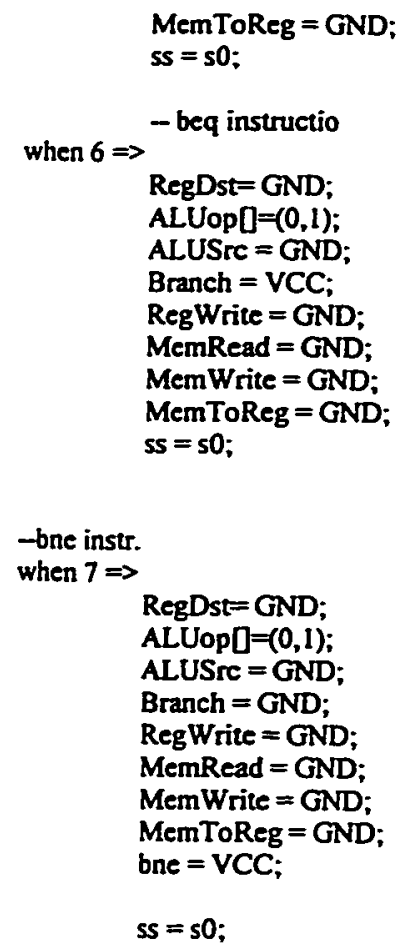

end case;

END CASE;

ss. $($ clk,reset $)=($ clk, reset $)$;

END;

$\%$

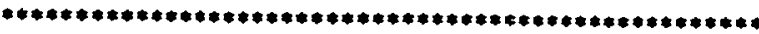

MODULE: 4BitAdder

INPUT: $\quad \operatorname{cin}, a[3 . .0], b[3 . .0]$

OUTPUT: $s[3 . .0]$,cout

$* * * * * * * * * * * * * * * * * * * * * * * * * * * * * * * * * * * * * * * * * * * * * * * * * * * *$

$\%$

SUBDESIGN 4BitAdder

(cin, a[3..0], b[3..0]: INPUT;

s[3..0],cout: OUTPUT; )

VARIABLE

$c 1, c 2, c 3, c 4: N O D E$;

$g 1, g 2, g 3, g 4:$ NODE;

pl, p2, p3, p4: NODE;

BEGIN

\%create the propagate, $p$, of the bits\%

$\mathrm{pl}=\mathrm{a}[0] \mathrm{Sb}$ [0];

$\mathrm{p} 2=\mathrm{a}[1] \mathrm{b}[1]$;

$\mathrm{p} 3=\mathrm{a}[2] \mathrm{S}$ b[2];

$p 4=a[3] S$ b[3]; 
$\%$ create the generator, $q$, of the bits $\%$

$\mathrm{gl}=\mathrm{a}[0] \& \mathrm{~b}[0] ;$

$\mathrm{g} 2=\mathrm{a}[1] \&$ b $[1]$;

$\mathrm{g} 3=\mathrm{a}[2] \& \mathrm{~b}[2]$

$\mathrm{g} 4=\mathrm{a}[3] \& \mathrm{~b}[3]$;

\%creare the carry look ahead generator\%

$\mathrm{cl}=\mathrm{cin}$;

$\mathrm{c} 2=(\mathrm{g} I \#(\mathrm{p} 1 \& \mathrm{cl}))$;

c3 $=(\mathrm{g} 2 H(\mathrm{p} 2 \& \mathrm{gl}) \#(\mathrm{p} 2 \& \mathrm{pl} \mid \mathrm{cl} \mathrm{l}))$;

$c 4=(g 3 \#(p 3 \& g 2) \#(p 3 \& p 2 \& g 1) \#(p 3 \& p 2 \& p 1 \& c 1)) ;$

$s[0]=\mathrm{clSpl}$;

$\%$ produce the sum and the $0 / p . . \%$

$s[1]=c 25 p 2$

$s[2]=\mathrm{c} 3 \mathrm{Sp} 3$;

$s[3]=\mathrm{c} 45 \mathrm{p} 4$;

END;

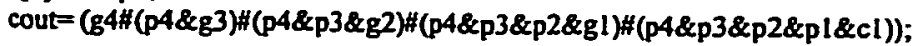

$\%$

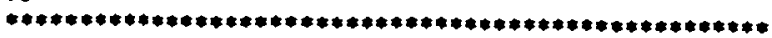

MODULE:

16BitALU

INPUT: $\quad a[15.0], b[15.0]$, Sel[3..0]

OUTPUT: F[15..0], $S[3 . .0]$

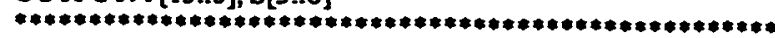

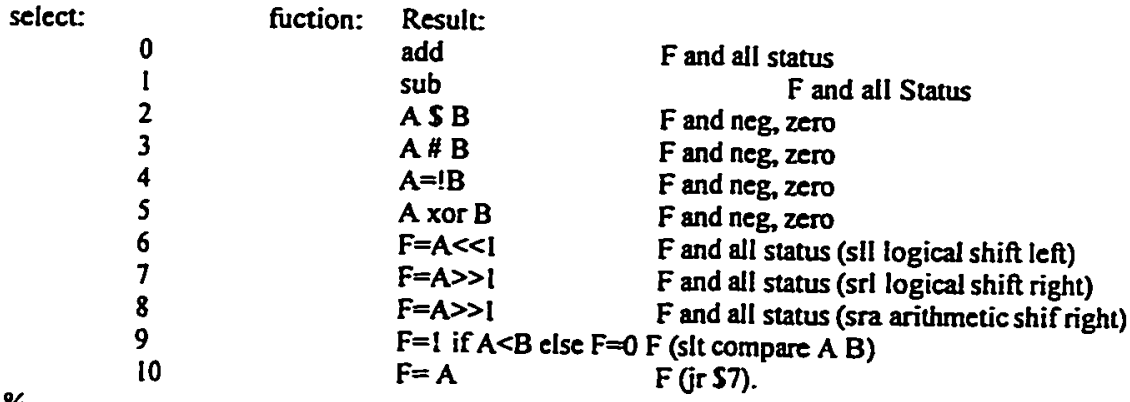

$\%$

FUNCTION 4BilAdder (cin, a[3..0], b[3..0])

RETURNS(s[3..0],cout);

\%FUNCTION RegFile(\%

SUBDESIGN I6BITALU

(a[15..0], b[15..0], Sel[3..0]: NPUT;

F[I5..0], S[3..0]: OUTPUT;

)

VARIABLE

U1, U2, U3, U4: 4BiLAdder,

BEGIN

CASE Sel[3..0] IS 
\%Adding two numbers.\%

WHEN $0 \Rightarrow$

\%assign UI's input a[3..0] $=a[3 . .0]$, the input from the register file\%

Ul. cin = GND;

$\% U I ' s$ cin $=$ the input cin.\%

U1.a[3..0]=a[3..0];

\%assign UI's input $a[3 . .0]=a[3 . .0]$, the input from the register file\%

Ul .b[3..0] $=b[3 . .0]$;

\%assign UI's input a[3.0] = a[7..4], the input from the register file\% U2.cin = UI .cout; \%casecading UI and U2.\%

U2.a[3..0 $=a[7 . .4]$;

\%assign Ul's input a[3...0] $=b[7 . .4]$, the input from the register file\%

$\mathrm{U} 2 . b[3 . .0]=b[7 . .4]$;

\%assign UI's input $a[3 . .0]=a[11 . .8]$, the input from the register file\%

U3.cin = U2. cout; $\%$ U3's cin $=$ the cout of $u 2 . \%$

$\mathrm{U} 3 . \mathrm{a}[3 . .0]=\mathrm{a}[11 . .8 \mathrm{~B}$;

$\%$ assign Ul's inputa[3..0] $=b[11 . .8]$, the input from the register file\% U3.b[3..0] $=$ b[11..8];

\%assign Ul's input $a[3 . .0]=a[15 . .12]$, the input from the register file\% U4. cin $=$ U3.cout; \%casecading U3and U4.\%

U4.a[3..0] $=a[15 . .12]$;

$\%$ assign Ul's input $a[3 . .0]=b[15 . .12]$, the input from the register file\% U4.b[3..0] $=b[15 . .12]$;

\%Now produce the Function bits and the status.\%

$F[3.0]=U 1 . S[3 . .0] ;$

$F[7.4]=U 2 . S[3 . .0]$;

$F[11.8]=U 3 . S[3.0]$;

$F[15 . .12]=U 4 . S[3 . .0]$;

$S[0]=U 4$. cout;

\%STATUS:Carty cout\%

$\%$ These if statements handles the overflow condition.\%

if $((\mathrm{a}[15 . .0]>0) \&(\mathrm{~b}[15 . .0]>0) \&(\mathrm{~F}[15 . .0]<0))$ then $S[1]=V C C$;

elsif $((\mathrm{a}[15 . .0]<0) \&(\mathrm{~b}[15.0]<0) \&(\mathrm{~F}[15 . .0]>0))$ then

else $\mathrm{S}[\mathrm{I}]=\mathrm{VCC}$

end if;

$S[1]=$ GND;

$S[2]=$

!(F[0]\#F[1]\#F[2]\#F[3]\#F[4]\#F[5]\#F[6]\#F[7]\#F[8]\#F[9]\#F[10]\#F[11]\#F[12]\#F[13]\#F[14]\#F[15]);\%STATUS: ZERO\% $S[3]=F[7]$;

$\%$ Subtracting two numbers.\%

WHEN $1 \Rightarrow$

\%assign UI's input $a[3 . .0]=a[3 . .0]$, the input from the register file\%

Ul. cin $=$ VCC; $\%$ added $I$ and 2 's complement b.\%

Ul.a[3..0] $=a[3 . .0]$;

$\%$ assign UI's input $a[3 . .0]=a[3 . .0]$, the input from the register file\%

U1.b[3..0] $=$ !b[3.0];

\%assign U2's input $a[3.0]=a[7 . .4]$, the input from the register file\% U2. cin = U1 . cout; \%casecading UI and U2.\%

U2.a[3...0 $=a[7 . .4]$;

\%assign UI's input $a[3 . .0]=b[7 . .4]$, the input from the register file\% $\mathrm{U} 2 . \mathrm{b}[3 . .0]=$ !b $[7 . .4]$

\%assign U2's input a[3..0] $=a[11 . .8]$, the input from the register file\% U3. cin = U2.cout; \%U3's cin = the cout of $12 \%$

$\mathrm{U} 3 . \mathrm{a}[3 . .0]=\mathrm{a}[11 . .8]$;

\%assign Ul's input $a[3 . .0]=b[11 \ldots 8]$, the input from the register file\% U3.b[3..0] $=$ !b $[11 . .8]$; 


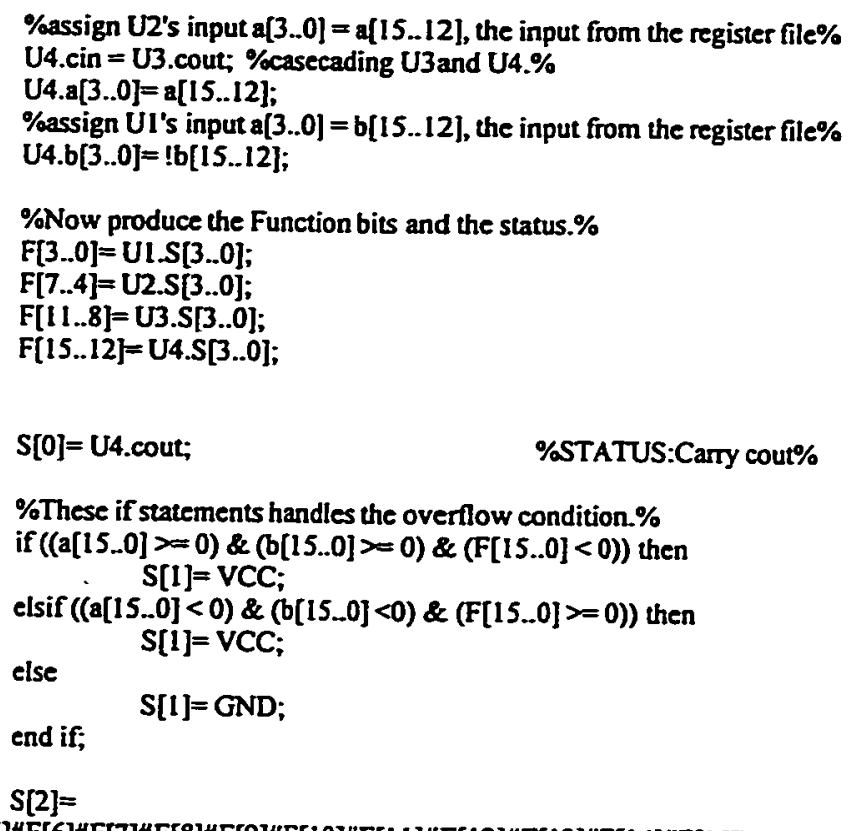

!(F[0]\#F[1]\#F[2]\#F[3]\#F[4]\#F[5]\#F[6]\#F[7]\#F[8]\#F[9]\#F[10]\#F[1 1]\#F[12]\#F[13]\#F[14]\#F[15]);\%STATUS: ZERO\% $S[3]=F[7]$ \%STATUS: NEGATTVE\%

WHEN $2 \Rightarrow$

\%perform $A$ and $B$ then check on zero flag or negative.\%

$F[15 . .0]=((a[15 . .0])$ AND $(b[15.0])) ;$
$S[2]=$

!(F[0]\#F[1]\#F[2]\#F[3]\#F[4]\#F[S] $S[2]=$

\%perform A\# B then check on zero flag or negative.\% $S[3]=F[15]$ $\mathrm{F}[15 . .0]=\mathrm{a}[15 . .0] \mathrm{OR} \mathrm{b}[15.0]$

! $F[0] \# F[1] \# F[2] \# F[3] \# F[4] \# F[5] \# F[6] \# F[7] \# F[8] \# F[9] \# F[10] \# F[11] \# F[12] \# F[13] \# F[14] \# F[15]) ; \% S T A T U S: Z E R O \%$ $S[3]=F[15]$

\%STATUS: NEGATTVE\%

WHEN $4 \Rightarrow$

$$
F[=! A D ;
$$

S[2] $=$
!(F[0]\#F[1] $\# \mathrm{~F}[2] \# \mathrm{~F}[3] \# \mathrm{~F}[4] \# \mathrm{~F}[5] \# \mathrm{~F}[6] \# \mathrm{~F}[7] \# \mathrm{~F}[8] \# \mathrm{~F}[9] \# \mathrm{~F}[10] \# \mathrm{~F}[11] \# \mathrm{~F}[12] \# \mathrm{~F}[13] \# \mathrm{~F}[14] \# \mathrm{~F}[15]) ; \%$ STATUS: ZERO\% $\mathrm{S}[3]=\mathrm{F}[\mathrm{I} 5]$

WHEN $S \Rightarrow$

$\% A$ xor $B t \%$

$\mathrm{F}[15 . .0]=\mathrm{A}]$ xor $\mathrm{BD}$;

$S[2]=$

!(F[0]\#F[1]\#F[2]\#F[3]\#F[4]\#F[5]\#F[6]\#F[7]\#F[8]\#F[9]\#F[1 0]\#F[1 1]\#F[12]\#F[13]\#F[14]\#F[15]);\%STATUS: ZERO\% WHEN $6 \Rightarrow$

\%local shift left 1 bit.\%

$F[15 . .1]=A[14 . .0]$;

$\mathrm{F}[0]=\mathrm{GND}$;

$S[2]=$

!(F[0]\#F[1]\#F[2]\#F[3]\#F[4]\#F[5]\#F[6]\#F[7]\#F[8]\#F[9]\#F[10]\#F[11]\#F[12]\#F[13]\#F[14]\#F[15]);\%STATUS:ZERO\%

WHEN 7 =>

\%logical shift right I bit\%

$F[14 . .0]=A[15 . .1]$; 
$F[15]=$ GND;

$S[2]=$

!(F[0]\#F[1]\#F[2]\#F[3]\#F[4]\#F[5]\#F[6]\#F[7]\#F[8]\#F[9]\#F[10]\#F[1 1 ]\#F[12]\#F[13]\#F[14]\#F[15]);\%STATUS: ZERO\%

WHEN $8 \Rightarrow$

\%arithmetic shift right 1 bit.\%

$F[13 . .0]=A[14 . .1]$

$F[15]=A[15]$;

$F[14]=$ GND;

$S[2]=$

!(F[0]\#F[1]\#F[2]\#F[3]\#F[4]\#F[5]\#F[6]\#F[7]\#F[8]\#F[9]\#F[10]\#F[1 1 ]\#F[12]\#F[13]\#F[14]\#F[15]);\%STATUS: ZERO\%

WHEN $9 \Rightarrow$

\%compare A B \%

IF $\mathrm{A}[<\mathrm{BD}$ then

else

FD= VCC;

$F[=A[]$

END IF;

FD= GND;

END;

END CASE;

$\%$

MODULE: 16BitAdder

INPUT: a[15..0], 15[3..0]

OUTPUT: F[15..0]

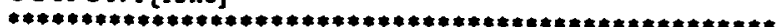

$\%$

FUNCTION 4BitAdder (cin, a[3..0], b $[3 . .0])$

RETURNS(s[3..0],cout);

\%FUNCTION RegFile(\%

SUBDESIGN 16 bitadder

(a[15..0], b[15..0]: INPUT;

F[15..0]: OUTPUT;

)

VARIABLE

U1, U2, U3, U4: 4BitAdder,

BEGIN

\%assign Ul's input $a[3 . .0]=a[3 . .0]$, the input from the register file\%

$\mathrm{UI}$.cin = GND;

$\%$ Ul's cin = the input cin.\%

U1 .a[3..0] $=a[3 . .0]$

\%assign UI's input $a[3 . .0]=a[3 . .0]$, the input from the register file\%

UI .b $[3 . .0]=b[3 . .0]$;

\%assign UI's input a[3..0] $=a[7 . .4]$, the input from the register file\%

U2.cin = U1 .cout; \%casecading UI and U2.\%

U2. $a[3 . .0]=a[7 . .4]$

\%assign UI's input $a[3 . .0]=b[7 . .4]$, the input from the register file\%

$\mathrm{U} 2 . \mathrm{b}[3 . .0]=b[7 . .4]$;

\%assign UI's input a[3..0] $=a[11 . .8]$, the input from the register file\% U3. $\operatorname{cin}=$ U2. cout; $\%$ U3's cin = the cout of $u 2 . \%$

U3. $a[3 . .0]=a[11 . .8]$;

\%assign U I's input $a[3 . .0]=b[11 . .8]$, the input from the register file\%

U3.b[3..0]= b[11..8]; 
\%assign UI's input a[3..0] $=a[15 . .12]$, the input from the register file\% U4.cin = U3.cout; \%casecading U3and U4.\%

U4.a[3..0] $=\mathrm{a}[15 . .12]$;

\%assign Ul's input $a[3 . .0]=b[15 . .12]$, the input from the register file\% U4.b[3..0] $=b[15 . .12]$;

\%Now produce the Function bits and the status.\%

$\mathrm{F}[3 . .0]=\mathrm{U} 1 . \mathrm{S}[3 . .0]$;

$F[7.4]=U 2 . S[3 . .0]$

$F[11 . .8]=U 3 . S[3 . .0]$

END;

$F[15 . .12]=U 4 . S[3 . .0]$; 

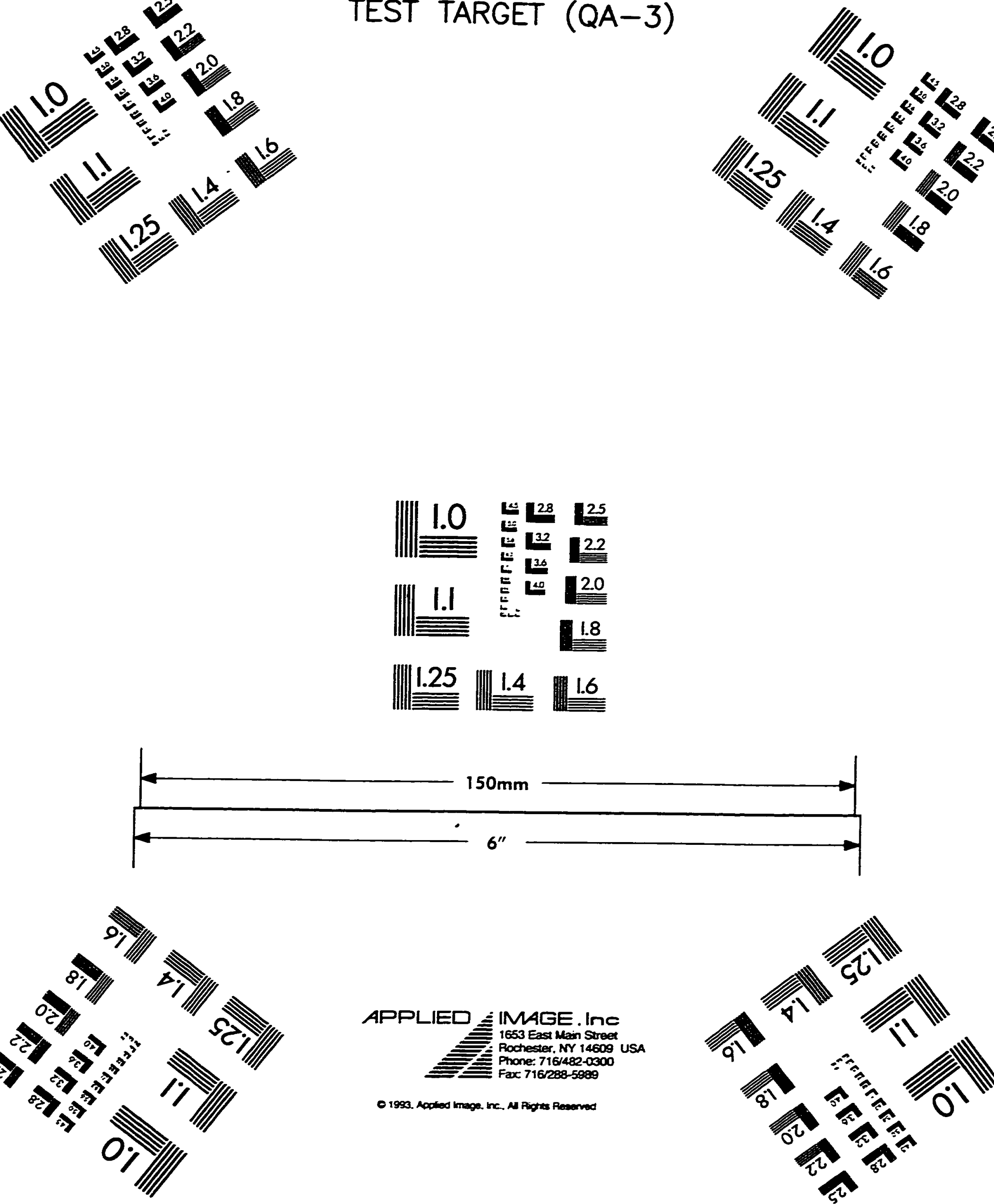

APPLIED ミIMAGE. InC

1653 Eass Main Street

Rochester. NY 14609 USA

三

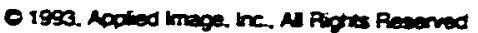

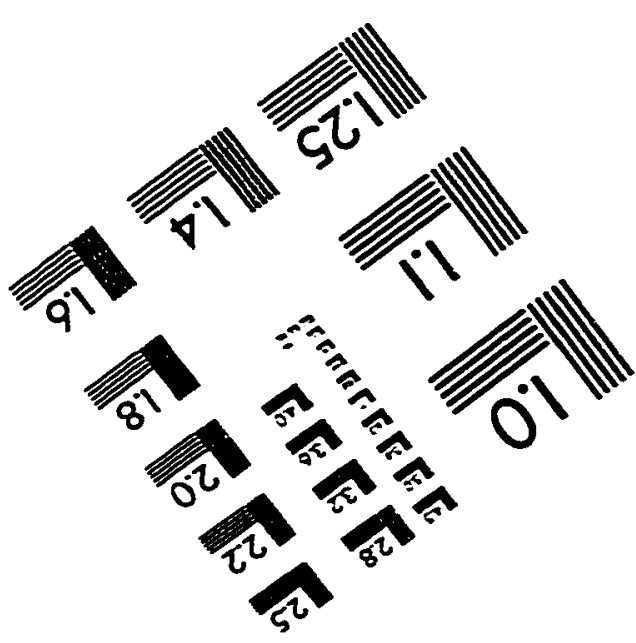

